\title{
On a free boundary problem for viscous incompressible flows
}

\author{
YASUNORI MAEKAWA ${ }^{\dagger}$ \\ Department of Mathematics, Hokkaido University, Sapporo, 060-0810, Japan
}

[Received 22 August 2006 and in revised form 19 April 2007]

\begin{abstract}
We shall discuss a free boundary problem for viscous incompressible fluids which is motivated by the phase transition of materials in a flowing fluid. The problem is formulated as the coupled Stokes/mean curvature equations. Our model is also regarded as the relaxation of a two-phase free boundary problem with surface tension on the interface. We shall construct a unique time-local solution of the problem by establishing the optimal regularity of the velocity field in the tangential directions to the interface.
\end{abstract}

\section{Introduction and formulation}

We are interested in the following free boundary problem for viscous incompressible flows. We consider the Stokes system:

$$
\left\{\begin{array}{l}
\partial_{t} u-\Delta u+\nabla p=\sigma_{1} H \nu \mathcal{H}_{\left\llcorner\Gamma_{t}\right.}^{n-1}, \quad 0<t \leqslant T, x \in \mathbb{R}^{n}, \\
\nabla \cdot u=0, \quad 0<t \leqslant T, \quad x \in \mathbb{R}^{n}, \\
u(0, x)=u_{0}(x), \quad x \in \mathbb{R}^{n},
\end{array}\right.
$$

where $u=\left(u_{1}, \ldots, u_{n}\right)$ and $p$ are unknown velocity and pressure fields, respectively. The symbol $\Gamma_{t}$ represents an unknown free interface evolving from the initial interface $\Gamma_{0}$ which is the boundary of a bounded domain $\Omega_{0}$. The positive constant $\sigma_{1}$ represents the surface tension, and $H, v$ are the mean curvature and the exterior unit normal vector of $\Gamma_{t}$, respectively. The symbol $\mathcal{H}_{\left\llcorner\Gamma_{t}\right.}^{n-1}$ means the $(n-1)$-dimensional Hausdorff measure restricted to $\Gamma_{t}$, i.e.,

$$
\left(f, \mathcal{H}_{\left\llcorner\Gamma_{t}\right.}^{n-1}\right)=\int_{\Gamma_{t}} f(y) \mathcal{H}^{n-1}(\mathrm{~d} y), \quad \forall f \in C_{0}\left(\mathbb{R}^{n}\right),
$$

where $C_{0}\left(\mathbb{R}^{n}\right)$ is the class of continuous functions with compact support, and (, ) is a pairing when we regard $\mathcal{H}_{\left\llcorner\Gamma_{t}\right.}^{n-1}$ as a linear functional on $C_{0}\left(\mathbb{R}^{n}\right)$.

We assume that the free interface is given by $\Gamma_{t}=\left\{x\left(t, x_{0}\right) \in \mathbb{R}^{n} ; x_{0} \in \Gamma_{0}\right\}$ where $x\left(t, x_{0}\right)$ is the solution of the ODE

$$
\left\{\begin{array}{l}
\frac{\mathrm{d} x(t)}{\mathrm{d} t}=u(t, x(t))+\sigma_{2} H(t, x(t)) v(t, x(t)), \quad 0<t \leqslant T, \\
x(0)=x_{0} \in \Gamma_{0}
\end{array}\right.
$$

where $\sigma_{2}$ is a fixed positive constant.

\footnotetext{
${ }^{\dagger}$ E-mail: yasunori@math.sci.hokudai.ac.jp
} 
The right hand side of the first equation in (FBP1) is the free boundary condition taken into account in weak sense. That is, the term $\sigma_{1} H \nu \mathcal{H}_{\left\llcorner\Gamma_{t}\right.}^{n-1}$ is formally equivalent to the free boundary condition

$$
\left[\left(-p \delta_{i j}+\partial_{j} u_{i}+\partial_{i} u_{j}\right)_{1 \leqslant i, j \leqslant n}\right]_{\Gamma_{t}} v=\sigma_{1} H v,
$$

where $[\cdot]_{\Gamma_{t}}$ is the jump across the interface $\Gamma_{t}$.

Our problem is motivated by the phase transition of materials in a flowing fluid. That is, the motion of the phase is not only governed by its mean curvature but also convected by the fluid velocity. The motion of the fluid is also influenced by the interface, which is represented by the free boundary condition. The phase transition with convection effects has been numerically well investigated. In [8], M. E. Gurtin, D. Polignore and J. Viñals considered the coupled NavierStokes/Cahn-Hilliard equations. In T. Blesgen [2] and C. Liu and J. Shen [12], the coupled NavierStokes/Allen-Cahn equations are studied. In fact, the above papers ([8], [2], [12]) deal with the case of two-phase binary fluids. In this paper, however, we consider the case of one fluid for simplicity.

Our model is also related to the following two-phase Stokes flow problem (in weak form):

$$
\begin{aligned}
& \left\{\begin{array}{l}
\partial_{t} u-\nabla \cdot T(\kappa D u, p)=\sigma_{1} H \nu \mathcal{H}_{\llcorner}^{n-1}, \quad 0<t \leqslant T, x \in \mathbb{R}^{n}, \\
\nabla \cdot u=0, \quad 0<t \leqslant T, x \in \mathbb{R}^{n}, \\
u(0, x)=u_{0}(x), \quad x \in \mathbb{R}^{n},
\end{array}\right. \\
& \left\{\begin{array}{l}
\frac{\mathrm{d} x(t)}{\mathrm{d} t}=u(t, x(t)), \quad 0<t \leqslant T, x(t) \in \Gamma_{t}, \\
x(0)=x_{0} \in \Gamma_{0},
\end{array}\right.
\end{aligned}
$$

where $T(\kappa D u, p):=2 \kappa_{1} \chi_{\Omega_{t}} D u+2 \kappa_{2}\left(1-\chi_{\Omega_{t}}\right) D u-p I$ is the stress tensor, $2 D u=\left(\partial_{j} u_{i}+\right.$ $\left.\partial_{i} u_{j}\right)_{1 \leqslant i, j \leqslant n}$ is the deformation tensor, $\kappa_{i}>0$ are the viscosity coefficients of the fluids, and $\Omega_{t}$ is a bounded domain with $\Gamma_{t}=\partial \Omega_{t}$. The characteristic function $\chi_{\Omega_{t}}$ is defined by $\chi_{\Omega_{t}}(x)=1$ if $x \in \Omega_{t}$ and $\chi_{\Omega_{t}}(x)=0$ if $x \in \mathbb{R}^{n} \backslash \Omega_{t}$.

Our problem can be regarded as the relaxation of the problem $(\mathrm{TP})$, since the viscosities and the densities of the two fluids are assumed to be the same and the term $\sigma_{2} \mathrm{Hv}$ in the kinematic boundary condition has a regularizing effect for the interface. Such relaxation in the kinematic boundary condition is used in the level set methods in numerical analysis; see Y. C. Chang, T. Y. Hou, B. Merriman and S. Osher [3]. The advantage of this method (or the phase-field method in [8], [12], [2]) is that one can capture the interface even when it develops singularities such as merging and reconnection.

Since $u$ is divergence free in the whole space, from (FBP1) we have

$$
\partial_{t} u-\Delta u=\mathbf{P} \sigma_{1} H \nu \mathcal{H}_{\left\llcorner\Gamma_{t}\right.}^{n-1},
$$

where $\mathbf{P}=\left(R_{i} R_{j}\right)_{1 \leqslant i, j \leqslant n}+I$ is the Helmholtz projection, and $R_{j}=\partial_{j}(-\Delta)^{-1 / 2}$ is the Riesz transformation. One can check that the term $\mathbf{P} \sigma_{1} H \nu \mathcal{H}_{\llcorner}^{n-1} \Gamma_{t}$ is well-defined at least in the class of tempered distributions if the hypersurface $\Gamma_{t}$ is a smooth boundary of a bounded domain.

In this paper, we shall construct the velocity field as a mild solution of the equation $(1.2)$, that is, the integral equation associated with $(1.2)$. Thus we shall consider the system

$$
\left\{\begin{array}{l}
u(t)=e^{t \Delta} u_{0}+\int_{0}^{t} e^{(t-s) \Delta} \mathbf{P} \sigma_{1} H \nu \mathcal{H}_{\left\llcorner\Gamma_{s}\right.}^{n-1} \mathrm{~d} s, \\
\mathrm{BC} .
\end{array}\right.
$$


Here, $e^{t \Delta}$ is the heat semigroup; see Section 4 for details. We assume that $u_{0}$ belongs to the class $C^{\alpha}\left(\mathbb{R}^{n}\right)$ of $\alpha$-Hölder continuous functions and $\Gamma_{0}$ is a $C^{2+\alpha}$ hypersurface for some $\alpha \in(0,1)$. Our aim is to construct a pair $\left(u,\left\{\Gamma_{t}\right\}_{0 \leqslant t \leqslant T}\right)$ solving $(\mathrm{FBP})$ with initial data $\left(u_{0}, \Gamma_{0}\right)$.

We say that a family of hypersurfaces $\left\{\Gamma_{t}\right\}_{0 \leqslant t \leqslant T}$ belongs to $C^{1,2+\alpha}$ when the signed distance function of $\Gamma_{t}$ belongs to $C^{1,2+\alpha}$ in a neighborhood of $\left\{\Gamma_{t}\right\}_{0 \leqslant t \leqslant T}$. A precise definition will be given in Section 3.

Now the main result of this paper is as follows.

THEOREM 1.1 (Existence and uniqueness) Let $\alpha \in(0,1)$. Assume that $u_{0} \in C^{\alpha}\left(\mathbb{R}^{n}\right)$ with $\nabla \cdot u_{0}=0$ and $\Omega_{0}$ is a bounded domain with $C^{2+\alpha}$ boundary. Let $\Gamma_{0}=\partial \Omega_{0}$. Then there exists a positive $T$ such that there is a unique solution $\left(u,\left\{\Gamma_{t}\right\}_{0 \leqslant t \leqslant T}\right)$ solving $\overline{\mathrm{FBP}}$ with initial data $\left(u_{0}, \Gamma_{0}\right)$ and such that $u \in C^{\alpha / 2, \alpha}\left([0, T] \times \mathbb{R}^{n}\right)$ and $\left\{\Gamma_{t}\right\}_{0 \leqslant t \leqslant T}$ belongs to $C^{1,2+\alpha}$.

REMARK 1.1 Let us make a remark on the pressure term. The pressure $p$ is reconstructed by the formula

$$
p(t, x)=-(-\Delta)^{-1} \nabla \cdot \sigma_{1} H \nu \mathcal{H}_{\llcorner}^{n-1}=-\sigma_{1} \nabla_{x} \cdot \int_{\Gamma_{t}} E(x-y) H \nu(t, y) \mathcal{H}^{n-1}(\mathrm{~d} y),
$$

where $E(x)$ is the Newton potential: $E(x)=-(2 \pi)^{-1} \log |x|$ when $n=2, E(x)=C(n)|x|^{2-n}$ when $n \geqslant 3$. The derivative $\nabla_{x}$ is interpreted in the sense of distributions. By similar calculations to those in Proposition 4.1. we can show that $p$ belongs at least to $L^{\infty}\left(0, T ; L^{r}\left(\mathbb{R}^{n}\right)\right)$ for $r \in(1, \infty)$.

As far as the author knows, there are few mathematical results for the free boundary problems in the presence of the term $\sigma_{2} H v$ in $(\mathrm{BC})$. But under the kinematic boundary condition $(\overline{\mathrm{BC}})$, there is much literature on free boundary problems for viscous incompressible (Navier-Stokes) flows with or without surface tension. For example, I. Sh. Mogilevskiı̌ and V. A. Solonnikov [15] showed the local well-posedness in Hölder spaces for one-phase flow problems; see also V. A. Solonnikov [23]. I. V. Denisova [4] and N. Tanaka [25] studied two-phase flow problems in Sobolev-Slobodetskiı̌ spaces. It is known that the global solvability holds near the equilibrium states for one- or two-phase flow problems; see M. Padula and V. A. Solonnikov [19] and N. Tanaka [24].

In these papers ([15], [23], [4], [25], [19], [24]), regular solutions are considered and Lagrangian coordinates are used in order to reduce the problem to the case of a fixed domain. But in our problem, such reduction is less useful because of the term $\sigma_{2} H v$ in $(\mathrm{BC})$. So we shall deal with the equation directly as in the formulation ( $\mathrm{FBP})$, and the free boundary condition appears in the layer potential term. Although the term $\sigma_{2} H v$ could lead to more complicated interactions between the interface and the fluid velocity, we have the mathematical advantage that we do not need the compatibility conditions between the boundary data and initial data. We remark that such compatibility conditions are required in the above papers.

Let us comment on weak solutions of two-phase flow problem. Y. Giga and S. Takahashi [7] studied two-phase Stokes flows, and A. Nouri, F. Poupaud and Y. Demay [17] studied multi-phase flows. Both papers deal with the case without surface tension. In P. I. Plotnikov [20], G. Nespoli and R. Salvi [16], and H. Abels [1], the case with surface tension is discussed. However, if surface tension is present, the existence of weak solutions is still open even for the Stokes flow, and only measure-valued varifold solutions or varifold solutions are obtained; see [20], [1] for details.

Now let us state the main idea and the outline of the proof of the main theorem. As the first step, for a given $u$ in an appropriate class of functions, we shall construct a family of hypersurfaces evolving by the equation in $(\mathrm{BC})$. Since it is regarded as the mean curvature equation with a 
convection term, we will follow the argument of L. C. Evans and J. Spruck [5] (see also A. Lunardi [13] and Y. Giga and S. Goto [6]), which reduces the equation to the one for the signed distance function of interfaces; see Section 3.

Next, for a given family of hypersurfaces, we estimate the layer potential term in the integral equation in (FBP). The main difficulty is that we cannot expect high regularity for $u$ in the whole space (for example, we cannot expect $u(t) \in C^{1+\alpha}\left(\mathbb{R}^{n}\right)$ in general) because of the jump relation of the layer potential. However, in order to obtain a unique regular solution for the convected mean curvature equation in (BC), we need the regularity of the convection term $u$ such as $u(t) \in C^{1+\alpha}\left(\mathbb{R}^{n}\right)$. To overcome these difficulties, we make use of the regularity for $u$ in the tangential directions to the interface. More precisely, if each interface has $C^{2+\alpha}$ regularity (and suitable regularity with respect to time), we have the optimal regularity for the layer potential term, namely $C^{1+\alpha}$ in the tangential directions. In order to establish this optimal regularity, we use the Hölder-Zygmund spaces; see Section 4.1 for details. The desired result in the main theorem is obtained by constructing a suitable contraction mapping for velocity fields; see Section 5 .

This paper is organized as follows. In Section 2, we give the definitions of the function spaces we use. In Section 3, we solve the mean curvature equation with a convection term. In Section 4.1, we establish the estimates for the layer potential term in (FBP). In Section 4.2, we comment on the mild solution of the Navier-Stokes equation with the layer potential term. In that section, we shall also give the outline of the proof for the local well-posedness of the Navier-Stokes equation when the layer potential term is given. The proof is the usual contraction argument by T. Kato [10]. We will see that the velocity also has the fine regularity in the tangential directions to $\Gamma_{t}$ even in the case of the Navier-Stokes flow. In Section 5, we shall construct a suitable contraction mapping and obtain the desired results. In our strategy, the estimates for the layer potential by using local coordinate transforms play an essential role: As their proof is somewhat lengthy, some parts of it are given in Appendix; see Section 6.

REMARK 1.2 When the fluid is described by the Navier-Stokes equation, the associated integral equation becomes

$$
u(t)=e^{t \Delta} u_{0}-\int_{0}^{t} e^{(t-s) \Delta} \mathbf{P} \nabla \cdot u \otimes u \mathrm{~d} s+\int_{0}^{t} e^{(t-s) \Delta} \mathbf{P} \sigma_{1} H \nu \mathcal{H}_{\left\llcorner\Gamma_{s}\right.}^{n-1} \mathrm{~d} s .
$$

Our result can be extended to this case, but its proof becomes complicated, especially when one constructs a contraction mapping for the free boundary problem. So in this paper, we consider the case of the Stokes flow for simplicity.

REMARK 1.3 In Section 3, we construct hypersurfaces by using the signed distance function. But this is not the only method to obtain the required results. Indeed, the method using the Hanzawa transformation would give a slightly simpler proof; see E. Hanzawa [9]. Especially, in the method of the signed distance function, we have to check that the solution $v$ of the associated equation satisfies the condition $|\nabla v| \equiv 1$, which is skipped in the use of the Hanzawa transformation. I would like to thank the referee for suggesting this point.

\section{Function spaces and embedding properties}

First of all, we introduce several function spaces. Let $D$ be either $\mathbb{R}^{n}$ or an open set in $\mathbb{R}^{n}$ with uniformly $C^{2}$ boundary. Let $C(\bar{D})$ denote the Banach space of all continuous and bounded 
functions in $\bar{D}$, endowed with the sup norm. Let $C^{m}(\bar{D})$ denote the set of all $m$ times continuously differentiable functions in $D$, with derivatives up to order $m$ bounded and continuously extendable up to the boundary. The norm of $C^{m}(\bar{D})$ is defined as

$$
\|f\|_{C^{m}(\bar{D})}:=\sum_{0 \leqslant k \leqslant m}\left\|\partial_{x}^{k} f\right\|_{C(\bar{D})}, \quad\left\|\partial_{x}^{k} f\right\|_{C(\bar{D})}:=\sum_{|\theta|=k}\left\|\partial_{x}^{\theta} f\right\|_{C(\bar{D})} .
$$

Here, $\theta=\left(\theta_{1}, \ldots, \theta_{n}\right)$ is a multi-index. We recall that $C([a, b] \times \bar{D})$ is the space of all continuous and bounded functions in $[a, b] \times \bar{D}$, endowed with the norm

$$
\|f\|_{C([a, b] \times \bar{D})}\left(=\|f\|_{\infty}\right):=\sup _{(t, x) \in[a, b] \times \bar{D}}|f(t, x)| .
$$

For $0<\alpha<1$, we denote by $C^{0, \alpha}([a, b] \times \bar{D})$ (respectively, $C^{\alpha / 2,0}([a, b] \times \bar{D})$ ) the space of continuous functions that are $\alpha$-Hölder continuous with respect to the space variables (respectively, $\alpha / 2$-Hölder continuous with respect to time), i.e.,

$$
\begin{gathered}
C^{0, \alpha}([a, b] \times \bar{D}):=\left\{f \in C([a, b] \times \bar{D}) ; f(t, \cdot) \in C^{\alpha}(\bar{D}), t \in[a, b]\right\}, \\
\|f\|_{C^{0, \alpha}([a, b] \times \bar{D})}\left(=\|f\|_{C^{0, \alpha}}\right):=\|f\|_{\infty}+\sup _{t \in[a, b]}[f(t, \cdot)]_{C^{\alpha}(\bar{D})},
\end{gathered}
$$

where

$$
[g]_{C^{\alpha}(\bar{D})}:=\sup _{x, y \in \bar{D}, x \neq y} \frac{|g(x)-g(y)|}{|x-y|^{\alpha}}
$$

and

$$
\begin{gathered}
C^{\alpha / 2,0}([a, b] \times \bar{D}):=\left\{f \in C([a, b] \times \bar{D}) ; f(\cdot, x) \in C^{\alpha / 2}([a, b]), x \in \bar{D}\right\}, \\
\|f\|_{C^{\alpha / 2,0}([a, b] \times \bar{D})}\left(=\|f\|_{C^{\alpha / 2,0}}\right):=\|f\|_{\infty}+\sup _{x \in \bar{D}}[f(\cdot, x)]_{C^{\alpha / 2}([a, b])},
\end{gathered}
$$

where

$$
[h]_{C^{\alpha / 2}([a, b])}:=\sup _{t, s \in[a, b], t>s} \frac{|h(t)-h(s)|}{(t-s)^{\alpha / 2}} .
$$

Moreover, the function spaces $C^{\alpha / 2, \alpha}([a, b] \times \bar{D}), C^{1,2}([a, b] \times \bar{D}), C^{1,2+\alpha}([a, b] \times \bar{D})$, $C^{1+\alpha / 2,2+\alpha}([a, b] \times \bar{D})$ are defined as follows:

$$
\begin{aligned}
& C^{\alpha / 2, \alpha}([a, b] \times \bar{D}):=C^{\alpha / 2,0}([a, b] \times \bar{D}) \cap C^{0, \alpha}([a, b] \times \bar{D}), \\
& \|f\|_{C^{\alpha / 2, \alpha}([a, b] \times \bar{D})}\left(=\|f\|_{C^{\alpha / 2, \alpha}}\right):=\|f\|_{C^{\alpha / 2,0}([a, b] \times \bar{D})}+\|f\|_{C^{0, \alpha}([a, b] \times \bar{D})} . \\
& C^{1,2}([a, b] \times \bar{D}):=\left\{f \in C([a, b] \times \bar{D}) ; \partial_{t} f, \partial_{i j} f \in C([a, b] \times \bar{D}), 1 \leqslant i, j \leqslant n\right\}, \\
& \|f\|_{C^{1,2}([a, b] \times \bar{D})}\left(=\|f\|_{\left.C^{1,2}\right)}:=\|f\|_{\infty}+\left\|\partial_{x} f\right\|_{\infty}+\left\|\partial_{t} f\right\|_{\infty}+\left\|\partial_{x}^{2} f\right\|_{\infty} .\right. \\
& C^{1,2+\alpha}([a, b] \times \bar{D}):=\left\{f \in C^{1,2}([a, b] \times \bar{D}) ; \partial_{t} f, \partial_{i j} f \in C^{0, \alpha}([a, b] \times \bar{D}), 1 \leqslant i, j \leqslant n\right\}, \\
& \|f\|_{C^{1,2+\alpha}([a, b] \times \bar{D})}\left(=\|f\|_{C^{1,2+\alpha}}\right):=\|f\|_{\infty}+\left\|\partial_{x} f\right\|_{\infty}+\left\|\partial_{t} f\right\|_{C^{0, \alpha}}+\left\|\partial_{x}^{2} f\right\|_{C^{0, \alpha}} .
\end{aligned}
$$


Let $X$ be a Banach space endowed with the norm $\|\cdot\|_{X}$. We denote by $C^{\alpha}([a, b] ; X)$ the Hölder space such that

$$
\begin{array}{r}
C^{\alpha}([a, b] ; X):=\left\{f \in C([a, b] ; X) ;[f]_{C^{\alpha}([a, b] ; X)}:=\sup _{t, s \in[a, b], t>s} \frac{\|f(t)-f(s)\|_{X}}{(t-s)^{\alpha}},\right. \\
\left.\|f\|_{C^{\alpha}([a, b] ; X)}:=\sup _{a \leqslant t \leqslant b}\|f(t)\|_{X}+[f]_{C^{\alpha}([a, b] ; X)}<\infty\right\} .
\end{array}
$$

Similarly,

$$
\begin{array}{r}
\operatorname{Lip}([a, b] ; X):=\left\{f \in C([a, b] ; X) ;[f]_{\operatorname{Lip}([a, b] ; X)}:=\sup _{t, s \in[a, b], t>s} \frac{\|f(t)-f(s)\|_{X}}{t-s},\right. \\
\left.\|f\|_{\operatorname{Lip}([a, b] ; X)}:=\sup _{a \leqslant t \leqslant b}\|f(t)\|_{X}+[f]_{\operatorname{Lip}([a, b] ; X)}<\infty\right\} .
\end{array}
$$

Now we state the embedding properties of the Hölder spaces defined above. The following lemma will be used freely in this paper.

LEMma 2.1 Let $0<\alpha<1$. Then there exists a positive constant $K_{\alpha}$ such that for any $f \in$ $C^{1,2+\alpha}([a, b] \times \bar{D})$,

$$
\begin{aligned}
\|f\|_{C^{1 / 2}\left([a, b] ; C^{1+\alpha}(\bar{D})\right)}+\|f\|_{\operatorname{Lip}\left([a, b] ; C^{\alpha}(\bar{D})\right)}+\left\|\partial_{x} f\right\|_{C^{(1+\alpha) / 2,0}}+\left\|\partial_{x}^{2} f\right\|_{C^{\alpha / 2,0}} & \leqslant K_{\alpha}\|f\|_{C^{1,2+\alpha}}
\end{aligned}
$$

Here, the constant $K_{\alpha}$ is independent of $b-a$ and $f$.

Proof. See A. Lunardi [13, Lemma 5.1.1].

\section{Motion of hypersurfaces by the mean curvature equations with convection}

In this section, we consider the hypersurfaces evolving in time via mean curvature with a convection term. Precisely, we shall construct a family of hypersurfaces $\left\{\Gamma_{t}\right\}_{0 \leqslant t \leqslant T}$ such that for $0 \leqslant t_{0} \leqslant$ $t \leqslant T, \Gamma_{t}=\left\{x\left(t, x_{0}\right) ; x_{0} \in \Gamma_{t_{0}}\right\}$ satisfies the ODE

$$
\left\{\begin{array}{l}
\frac{\mathrm{d} x(t)}{\mathrm{d} t}=-\frac{\sigma_{2}}{n-1}[\operatorname{div}(v(t, x(t)))] v(t, x(t))+u(t, x(t)), \quad t_{0} \leqslant t \leqslant T, \\
x\left(t_{0}\right)=x_{0} .
\end{array}\right.
$$

Here $v(t, x)$ is the exterior unit normal vector of $\Gamma_{t}, \sigma_{2}$ is a positive constant, and $u(t, x)$ is a continuous function on $[0, T] \times \mathbb{R}^{n}$. The mean curvature $H(t, x)$ of the surface $\Gamma_{t}$ is given by $H(t, x)=-\frac{1}{n-1} \operatorname{div} v(t, x)$. So if $u \equiv 0$, the above equation is the well-known mean curvature flow equation. To construct evolving hypersurfaces starting from a given smooth initial hypersurfaces, we will follow the arguments of L. C. Evans and J. Spruck [5]; see also A. Lunardi [13]. Let $\left\{\Gamma_{t}\right\}_{0 \leqslant t \leqslant T}$ be the evolving hypersurfaces such that each $\Gamma_{t}$ is the boundary of a bounded domain $\Omega_{t}$. We reduce the equation to an equation for the signed distance function

$$
d(t, x)= \begin{cases}\operatorname{dist}\left(x, \Gamma_{t}\right), & x \in \mathbb{R}^{n} \backslash \bar{\Omega}_{t}, \\ -\operatorname{dist}\left(x, \Gamma_{t}\right), & x \in \Omega_{t} .\end{cases}
$$


If $\Gamma_{t}$ is smooth, then $d(t, \cdot)$ is also smooth in each of the sets

$$
D^{+}:=\left\{x \in \mathbb{R}^{n} ; 0 \leqslant d(t, x)<\delta_{0}\right\} \quad \text { and } \quad D^{-}:=\left\{x \in \mathbb{R}^{n} ;-\delta_{0}<d(t, x) \leqslant 0\right\},
$$

provided $\delta_{0}>0$ and $T>0$ is small. Moreover, if $\delta_{0}$ is sufficiently small, for each $x \in D^{+}$there exists a unique $y \in \Gamma_{t}$ such that $d(t, x)=|y-x|$. The equation (3.1) implies that

$$
\begin{aligned}
d_{t}(t, x) & =\left\langle\frac{\mathrm{d} y}{\mathrm{~d} t}, \frac{y-x}{|y-x|}\right\rangle=\left\langle-\frac{\sigma_{2}}{n-1}[\operatorname{div} v(t, y)] v(t, y)+u(t, y), \frac{y-x}{|y-x|}\right\rangle \\
& =\frac{\sigma_{2}}{n-1} \operatorname{div} v(t, y)-u\left(t, x-d \nabla_{x} d(t, x)\right) \cdot \nabla_{x} d(t, x) .
\end{aligned}
$$

It is well-known that the eigenvalues of the Hessian $\nabla^{2} d(t, x)$ are given by

$$
\lambda_{i}=-\frac{\kappa_{i}(t, y)}{1-\kappa_{i}(t, y) d(t, x)}, \quad i=1, \ldots, n-1, \quad \lambda_{n}=0,
$$

where the $\kappa_{i}$ are the principal curvatures of the surface $\Gamma_{t}$. Since the mean curvature $H$ is defined as $H=\frac{1}{n-1} \sum_{i=1}^{n-1} \kappa_{i}$, we have

$$
d_{t}=\frac{\sigma_{2}}{n-1} f\left(d, \nabla^{2} d\right)-u(t, x-d \nabla d) \cdot \nabla d,
$$

where

$$
f(s, q)=\sum_{i=1}^{n} \frac{\lambda_{i}}{1-\lambda_{i} s}, \quad s \in \mathbb{R}, q \in \mathbb{R}_{S}^{n \times n}, \lambda_{i} s \neq 1 .
$$

Here the $\lambda_{i}$ are the eigenvalues of the symmetric matrix $q$. The same equation can be deduced for $x \in D^{-}$. Since $|d|$ is a distance function, the spatial gradient $\nabla d$ should have modulus 1 at any point. This provides a nonlinear first order boundary condition for $d$. So the equation 3.1) is reduced to the following fully nonlinear parabolic problem:

$$
\left\{\begin{array}{l}
\partial_{t} v=\frac{\sigma_{2}}{n-1} f\left(v, \nabla^{2} v\right)-u(t, x-v \nabla v) \cdot \nabla v, \quad t \geqslant 0, x \in \bar{D}, \\
|\nabla v|^{2}=1, \quad t \geqslant 0, x \in \partial D, \\
v(0, x)=d_{0}(x), \quad x \in \bar{D},
\end{array}\right.
$$

where $D=D^{+} \cup D^{-}=\left\{x \in \mathbb{R}^{n} ;-\delta_{0}<d_{0}(x)<\delta_{0}\right\}, d_{0}$ is the signed distance function from $\Gamma_{0}$, and $f$ is given as above. We choose $\delta_{0}$ so small that $\lambda_{i}\left(\nabla^{2} d_{0}\right) \delta_{0} \neq 1$ for each $i$, so $f$ is well-defined near the range of $\left(d_{0}(x), \nabla^{2} d_{0}(x)\right)$. Since $f(s, q)=\operatorname{Tr}\left(q(I-s q)^{-1}\right), f$ is analytic. Moreover, since $\operatorname{Tr}\left(\frac{\partial f}{\partial q}(s, q) A\right)=\operatorname{Tr}\left((I-s q)^{-2} A\right)$ for $A \in \mathbb{R}^{n \times n}$, we have, for $\xi \in \mathbb{R}^{n}$,

$$
\sum_{i, j=1}^{n} f_{q_{i j}}(s, q) \xi_{i} \xi_{j}=\operatorname{Tr}\left(\frac{\partial f}{\partial q}(s, q) \xi \otimes \xi\right)=\sum_{i=1}^{n} \frac{1}{\left(1-\lambda_{i} s\right)^{2}}\left\langle\xi, \bar{e}_{i}\right\rangle^{2},
$$

where $\left\{\bar{e}_{1}, \ldots, \bar{e}_{n}\right\}$ is an orthonormal basis in $\mathbb{R}^{n}$ such that each $\bar{e}_{i}$ is an eigenvector of $q$ with eigenvalue $\lambda_{i}$. Thus we have

$$
\sum_{i, j=1}^{n} f_{q_{i j}}(s, q) \xi_{i} \xi_{j} \geqslant \iota(s, q)|\xi|^{2}
$$

with $\iota(s, q)=\min _{1 \leqslant i \leqslant n}\left(1-\lambda_{i} s\right)^{-2}$. 
Set $g(p)=p^{2}-1$. In order to solve the equation 3.6 , we linearize the principal term $f\left(v, \nabla^{2} v\right)$ near the initial data $\left(d_{0}, \nabla^{2} d_{0}\right)$ and $g\left(\nabla d_{0}\right)$ near $\nabla d_{0}$. The existence and uniqueness result for this equation is proved by the general results for linear parabolic equations and the usual contraction arguments. Let $B\left(d_{0}, \nabla^{2} d_{0}\right)$ be a bounded open neighborhood of the set $\left\{\left(d_{0}(x), \nabla^{2} d_{0}(x)\right) \in \mathbb{R} \times\right.$ $\left.\mathbb{R}^{n \times n} ; x \in \bar{D}\right\}$ such that for each $(s, q) \in B\left(d_{0}, \nabla^{2} d_{0}\right)$, the function $f(s, q)$ is well-defined. Set

$$
\begin{aligned}
\iota & :=\inf \left\{\iota(s, q) ;(s, q) \in B\left(d_{0}, \nabla^{2} d_{0}\right)\right\}>0, \\
K_{f} & :=\sup \left\{\left|\frac{\partial^{\beta} f}{\partial s \partial q}(s, q)\right| ;(s, q) \in B\left(d_{0}, \nabla^{2} d_{0}\right),|\beta|=0,1,2\right\} .
\end{aligned}
$$

Fix $M>0$. We assume that the convection term $u(t, x)$ belongs to $\mathcal{U}_{M}$, the closed subset of $C^{0, \alpha}\left([0, T] \times \mathbb{R}^{n}\right)$ defined as

$$
\begin{aligned}
\mathcal{U}_{M}:=\left\{u(t, x) \in C^{0, \alpha}\left([0, T] \times \mathbb{R}^{n}\right) ; u(t, \cdot) \in C^{1+\alpha}\left(\mathbb{R}^{n}\right), \text { and } \sup _{0<t<T}\|u(t, \cdot)\|_{C^{\alpha}\left(\mathbb{R}^{n}\right)}\right. \\
\left.+\sup _{0<t<T} t^{(1-\alpha) / 2}\left\|\partial_{x} u(t, \cdot)\right\|_{C\left(\mathbb{R}^{n}\right)}+\sup _{0<t<T} t^{1 / 2}\left[\partial_{x} u(t, \cdot)\right]_{C^{\alpha}\left(\mathbb{R}^{n}\right)} \leqslant M\right\}
\end{aligned}
$$

The following proposition states the existence and uniqueness for the equation 3.6.

Proposition 3.1 Fix $M>0$. Let $\alpha \in(0,1)$. Assume that $\Omega_{0}$ is a bounded domain with uniformly $C^{2+\alpha}$ boundary and let $d_{0}$ be the signed distance function of $\Gamma_{0}=\partial \Omega_{0}$. Then there is some $T>0$ such that for any $u \in \mathcal{U}_{M}$, there exists a unique $v \in C^{1,2+\alpha}([0, T] \times \bar{D})$ solving (3.6).

Proof. By appropriate rescaling, we may assume that $\sigma_{2} /(n-1)=1$. For simplicity of notation,

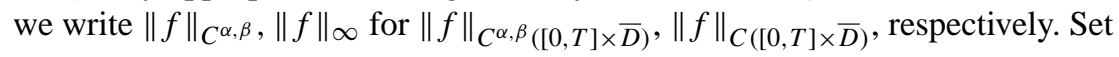

$$
\begin{aligned}
& \mathcal{A} v(t, x):=\sum_{i, j=1}^{n} f_{q_{i j}}\left(d_{0}(x), \nabla^{2} d_{0}(x)\right) \partial_{i j} v(t, x)+f_{s}\left(d_{0}(x), \nabla^{2} d_{0}(x)\right) v(t, x), \\
& \mathcal{B} v(t, x):=\sum_{i=1}^{n} g_{p_{i}}\left(\nabla d_{0}(x)\right) \partial_{i} v(t, x) .
\end{aligned}
$$

Let $R$ be a positive number to be determined later. We will find the solution in the set

$$
X:=\left\{v \in C^{1,2+\alpha}([0, T] \times \bar{D}) ; v(0, \cdot)=d_{0},\left\|v-d_{0}\right\|_{C^{1,2+\alpha}} \leqslant R\right\}
$$

as a fixed point of the operator $\Phi$ defined in $X$, where $\Phi(v)=w$ is the solution of the equation

$$
\left\{\begin{array}{l}
\partial_{t} w(t, x)=\mathcal{A} w+f\left(v, \nabla^{2} v\right)-\mathcal{A} v-u(t, x-v \nabla v) \cdot \nabla v, \quad 0 \leqslant t \leqslant T, x \in \bar{D} \\
\mathcal{B} w(t, x)=-g(\nabla v)+\mathcal{B} v, \quad 0 \leqslant t \leqslant T, x \in \partial D \\
w(0, x)=d_{0}(x), \quad x \in \bar{D}
\end{array}\right.
$$

Let $K_{\alpha}$ be the constant of Lemma 2.1. Then for all $v \in X$,

$$
\sum_{i, j=1}^{n}\left\|\partial_{i, j} v-\partial_{i, j} d_{0}\right\|_{\infty}+\left\|v-d_{0}\right\|_{\infty} \leqslant\left(K_{\alpha} T^{\alpha / 2}+T\right) R
$$


So $f\left(v, \nabla^{2} v\right)$ is well-defined in $X$ if we take $T$ so small that every $v \in X$ satisfies $\left(v(t, x), \nabla^{2} v(t, x)\right) \in B\left(d_{0}, \nabla^{2} d_{0}\right)$ for $(t, x) \in[0, T] \times \bar{D}$. The general results for linear parabolic equations guarantee that the equation has a unique solution in $C^{1,2+\alpha}([0, T] \times \bar{D})$. We shall show that for every $v_{1}, v_{2} \in X$,

$$
\left\|\Phi\left(v_{1}\right)-\Phi\left(v_{2}\right)\right\|_{C^{1,2+\alpha}} \leqslant C(R) T^{\alpha / 2}\left\|v_{1}-v_{2}\right\|_{C^{1,2+\alpha}} .
$$

Thus if $T$ is so small that $C(R) T^{\alpha / 2} \leqslant 1 / 2$ then $\Phi$ is a contraction mapping. We also have, for all $v \in X$,

$\left\|\Phi(v)-d_{0}\right\|_{C^{1,2+\alpha}} \leqslant\left\|\Phi(v)-\Phi\left(d_{0}\right)\right\|_{C^{1,2+\alpha}}+\left\|\Phi\left(d_{0}\right)-d_{0}\right\|_{C^{1,2+\alpha}} \leqslant R / 2+\left\|\Phi\left(d_{0}\right)-d_{0}\right\|_{C^{1,2+\alpha}}$.

The function $z=\Phi\left(d_{0}\right)-d_{0}$ is the solution of

$$
\left\{\begin{array}{l}
\partial_{t} z(t, x)=\mathcal{A} z(t, x)+f\left(d_{0}, \nabla^{2} d_{0}\right)-u\left(t, x-d_{0} \nabla d_{0}\right) \cdot \nabla d_{0}, \quad 0 \leqslant t \leqslant T, x \in \bar{D}, \\
\mathcal{B} z(t, x)=-g\left(\nabla d_{0}\right), \quad 0 \leqslant t \leqslant T, x \in \partial D, \\
z(0, x)=0, \quad x \in \bar{D} .
\end{array}\right.
$$

So $z$ satisfies the estimate

$$
\begin{aligned}
\|z\|_{C^{1,2+\alpha}} & \leqslant C\left(\left\|f\left(d_{0}, \nabla^{2} d_{0}\right)\right\|_{C^{\alpha}(\bar{D})}+\left\|u\left(\cdot, \cdot-d_{0} \nabla d_{0}\right) \cdot \nabla d_{0}\right\|_{C^{0, \alpha}([0, T] \times \bar{D})}+\left\|g\left(\nabla d_{0}\right)\right\|_{C^{1+\alpha}}\right) \\
& \leqslant C\left(\left\|d_{0}\right\|_{C^{2+\alpha}}, M\right),
\end{aligned}
$$

hence

$$
\left\|\Phi(v)-d_{0}\right\|_{C^{1,2+\alpha}} \leqslant R / 2+C\left(\left\|d_{0}\right\|_{C^{2+\alpha}}, M\right) .
$$

Thus for $R=2 C\left(\left\|d_{0}\right\|_{C^{2+\alpha}}, M\right), \Phi$ is a contraction mapping $X$ into itself, which implies that $\Phi$ has a unique fixed point in $X$. The uniqueness of the solution in $C^{1,2+\alpha}([0, T] \times \bar{D})$ follows easily, so we omit it.

Now let us prove the key estimate 3.14). Set $w=\Phi\left(v_{1}\right)-\Phi\left(v_{2}\right)$. Then $w$ is the solution of

$$
\left\{\begin{aligned}
& \partial_{t} w= \mathcal{A} w+f\left(v_{1}, \nabla^{2} v_{1}\right)-f\left(v_{2}, \nabla^{2} v_{2}\right)-\mathcal{A}\left(v_{1}-v_{2}\right) \\
&-u\left(t, x-v_{1} \nabla v_{1}\right) \cdot \nabla v_{1}+u\left(t, x-v_{2} \nabla v_{2}\right) \cdot \nabla v_{2}, \quad 0 \leqslant t \leqslant T, x \in \bar{D}, \\
& \mathcal{B} w= \mathcal{B}\left(v_{1}-v_{2}\right)-g\left(\nabla v_{1}\right)+g\left(\nabla v_{2}\right), \quad 0 \leqslant t \leqslant T, x \in \partial D, \\
& w(0, x)=0, \quad x \in \bar{D} .
\end{aligned}\right.
$$

From the optimal regularity for linear parabolic equations ([13, Theorem 5.1.21]), $w$ satisfies

$$
\|w\|_{C^{1,2+\alpha}} \leqslant C\left(\left\|h_{1}\right\|_{C^{0, \alpha}}+\left\|h_{2}\right\|_{C^{0, \alpha}}+\left\|h_{3}\right\|_{C^{(1+\alpha) / 2,1+\alpha}}\right),
$$

where

$$
\begin{aligned}
& h_{1}(t, x):=f\left(v_{1}(t, x), \nabla^{2} v_{1}(t, x)\right)-f\left(v_{2}(t, x), \nabla^{2} v_{2}(t, x)\right)-\mathcal{A}\left(v_{1}(t, x)-v_{2}(t, x)\right), \\
& h_{2}(t, x):=u\left(t, x-v_{1}(t, x) \nabla v_{1}(t, x)\right) \cdot \nabla v_{1}(t, x)-u\left(t, x-v_{2}(t, x) \nabla v_{2}(t, x)\right) \cdot \nabla v_{2}(t, x), \\
& h_{3}(t, x):=\mathcal{B}\left(v_{1}(t, x)-v_{2}(t, x)\right)-g\left(\nabla v_{1}(t, x)\right)+g\left(\nabla v_{2}(t, x)\right) .
\end{aligned}
$$


First we shall estimate $h_{1}$. By the definition of $\mathcal{A}$, we have

$$
\begin{aligned}
h_{1}(t, x)= & \int_{0}^{1}\left(f_{s}\left(\eta_{\tau}(t, x)\right)-f_{s}\left(\eta_{0}(x)\right)\right) \mathrm{d} \tau\left(v_{1}(t, x)-v_{2}(t, x)\right) \\
& +\sum_{i, j=1}^{n} \int_{0}^{1}\left(f_{q_{i, j}}\left(\eta_{\tau}(t, x)\right)-f_{q_{i, j}}\left(\eta_{0}(x)\right)\right) \mathrm{d} \tau\left(\partial_{i, j} v_{1}(t, x)-\partial_{i, j} v_{2}(t, x)\right),
\end{aligned}
$$

where

$$
\begin{aligned}
\eta_{\tau}(t, x) & :=\tau\left(v_{1}(t, x), \nabla^{2} v_{1}(t, x)\right)+(1-\tau)\left(v_{2}(t, x), \nabla^{2} v_{2}(t, x)\right) \in \mathbb{R} \times \mathbb{R}^{n \times n}, \\
\eta_{0}(x) & :=\left(d_{0}(x), \nabla^{2} d_{0}(x)\right) \in \mathbb{R} \times \mathbb{R}^{n \times n} .
\end{aligned}
$$

Clearly,

$$
\begin{aligned}
\left|\eta_{\tau}(t, x)-\eta_{0}(x)\right| & \leqslant 2\left(T+K_{\alpha} T^{\alpha / 2}\right) R, \\
\left|\eta_{\tau}(t, x)-\eta_{\tau}(t, y)\right| & \leqslant C\left(\left\|d_{0}\right\|_{C^{2+\alpha}}+R\right)|x-y|^{\alpha}, \\
\left|\eta_{0}(x)-\eta_{0}(y)\right| & \leqslant C\left\|d_{0}\right\|_{C^{2+\alpha}}|x-y|^{\alpha},
\end{aligned}
$$

where $C$ depends only on $n$, and $K_{\alpha}$ is the constant of Lemma 2.1. From these estimates and regularity assumptions on $f$, it is not difficult to deduce the estimates

$$
\begin{aligned}
\left|h_{1}(t, x)\right| & \leqslant C\left(T+K_{\alpha} T^{\alpha / 2}\right) R K_{f} T^{\alpha / 2}\left\|v_{1}-v_{2}\right\|_{C^{1,2+\alpha}}, \\
\left|h_{1}(t, x)-h_{1}(t, y)\right| & \leqslant C\left(\left\|d_{0}\right\|_{C^{2+\alpha}}+R+K_{\alpha}\right) K_{f} T^{\alpha / 2}\left\|v_{1}-v_{2}\right\|_{C^{1,2+\alpha}}|x-y|^{\alpha},
\end{aligned}
$$

hence

$$
\left\|h_{1}\right\|_{C^{0, \alpha}} \leqslant C\left(\left\|d_{0}\right\|_{C^{2+\alpha}}+R+K_{\alpha}\right) K_{f} T^{\alpha / 2}\left\|v_{1}-v_{2}\right\|_{C^{1,2+\alpha}},
$$

where $C$ depends only on $n$, and $K_{\alpha}, K_{f}$ are the constants of Lemma 2.1 and (3.9), respectively. The estimate of $h_{3}$ is similar:

$$
\left\|h_{3}\right\|_{C^{(1+\alpha) / 2,1+\alpha}} \leqslant C\left(\left\|d_{0}\right\|_{C^{2+\alpha}}, R, K_{\alpha}, K_{f}\right) T^{\alpha / 2}\left\|v_{1}-v_{2}\right\|_{C^{1,2+\alpha}} ;
$$

the details are omitted.

Next we shall estimate $h_{2}$. By the mean value theorem,

$$
\begin{aligned}
h_{2}(t, x) & =\int_{0}^{1}\left\langle-v_{1} \nabla v_{1}+v_{2} \nabla v_{2},\left(\nabla_{x} u\right)\left(t, \bar{\eta}_{\tau}(t, x)\right)\right\rangle \mathrm{d} \tau \cdot \nabla v_{1}+u\left(t, x-v_{2} \nabla v_{2}\right) \cdot\left(\nabla v_{1}-\nabla v_{2}\right) \\
& =: h_{2,1}(t, x)+h_{2,2}(t, x),
\end{aligned}
$$

where

$$
\bar{\eta}_{\tau}(t, x):=x-\tau v_{1} \nabla v_{1}(t, x)-(1-\tau) v_{2} \nabla v_{2}(t, x) .
$$

From the regularity assumption on $u$ and Lemma 2.1

$$
\begin{aligned}
\left|h_{2,1}(t, x)\right| & \leqslant\left(t+K_{\alpha} t^{(1+\alpha) / 2}\right)\left(R+\left\|d_{0}\right\|_{C^{2+\alpha}}\right)^{2}\left\|v_{1}-v_{2}\right\|_{C^{1,2+\alpha}} M t^{-(1-\alpha) / 2} \\
& \leqslant M\left(R+\left\|d_{0}\right\|_{C^{2+\alpha}}\right)^{2} T^{\alpha / 2}\left\|v_{1}-v_{2}\right\|_{C^{1,2+\alpha}},
\end{aligned}
$$


and

$$
\left|h_{2,2}(t, x)\right| \leqslant M K_{\alpha} T^{(1+\alpha) / 2}\left\|v_{1}-v_{2}\right\|_{C^{1,2+\alpha}} .
$$

Since $\left|\bar{\eta}_{\tau}(t, x)-\bar{\eta}_{\tau}(t, y)\right| \leqslant\left(1+4\left(R+\left\|d_{0}\right\|_{C^{2+\alpha}}\right)^{2}\right)|x-y|$, we easily have

$$
\left|h_{2,1}(t, x)-h_{2,1}(t, y)\right| \leqslant C\left(M, K_{\alpha}, R,\left\|d_{0}\right\|_{C^{2+\alpha}}\right) T^{\alpha / 2}\left\|v_{1}-v_{2}\right\|_{C^{1,2+\alpha}}|x-y|^{\alpha},
$$

and

$$
\left|h_{2,2}(t, x)-h_{2,2}(t, y)\right| \leqslant C\left(M, K_{\alpha}, R,\left\|d_{0}\right\|_{C^{2+\alpha}}\right) T^{\alpha / 2}\left\|v_{1}-v_{2}\right\|_{C^{1,2+\alpha}}|x-y|^{\alpha} .
$$

Thus the estimate (3.14) follows, and the proof of Proposition 3.1 is complete.

REMARK 3.1 From (3.16) in the proof above, the solution $v$ satisfies

$$
\|v\|_{C^{1,2+\alpha}([0, T] \times \bar{D})} \leqslant\left\|d_{0}\right\|_{C^{2+\alpha}}+2 C\left(\left\|d_{0}\right\|_{C^{2+\alpha}}, M\right) .
$$

We also see that the existence time of the solution does not depend on $u \in \mathcal{U}_{M}$.

We set $\Gamma_{t}:=\{x \in \bar{D} ; v(t, x)=0\}$ when $v$ is the solution of the equation (3.6) satisfying $v(0, x)=d_{0}(x)$. By arguing as in [13, Proposition 8.5.9], we see that the solution $v$ is such that $\partial_{i} v \in C^{1,2+\alpha}\left(\left[t_{1}, t_{2}\right] \times \overline{D^{\prime}}\right), i=1, \ldots, n$, for any open set $D^{\prime} \subset \subset D$ and $0<t_{1}<t_{2} \leqslant T$. Under this regularity condition, we can show that $\left\{\Gamma_{t}\right\}_{0 \leqslant t \leqslant T}$ is an evolving hypersurface satisfying the convected mean curvature equation 3.1. Precisely, we have the following proposition.

Proposition 3.2 Assume that the conditions in Proposition 3.1 are satisfied. Then the first derivatives of the solution $v$ satisfy the estimate

$$
\left\|\partial_{x} v\right\|_{C^{1,2+\alpha}\left(\left[t_{1}, t_{2}\right] \times \overline{\left.D^{\prime}\right)}\right.} \leqslant C\left(\frac{\left(t_{2}-t_{1}\right)^{1 / 2}}{t_{1}}+t_{1}^{-1 / 2}\right),
$$

where $C$ depends only on $n, \iota, \sigma_{2}, \alpha, K_{\alpha},\left\|d_{0}\right\|_{C^{2+\alpha}(\bar{D})}, M, K_{f}$, and dist $\left(D^{\prime}, \partial D\right)$. Moreover, for each $t>0, \Gamma_{t}$ is a $C^{3+\alpha}$ hypersurface and $\left\{\Gamma_{t}\right\}_{0 \leqslant t \leqslant T}$ is a unique family of $C^{2+\alpha}$ hypersurfaces evolving by the perturbed mean curvature equation (3.1) starting from $\Gamma_{0}$.

Proof. Again we may assume that $\sigma_{2} /(n-1)=1$. The assertion on the regularity of $\partial_{x} v$ follows by arguing as in [13, Proposition 8.5.6]. We omit the details. We shall only show the last assertion of the proposition. First we prove that $\left|\partial_{x} v\right| \equiv 1$. Set $w=\left|\partial_{x} v\right|^{2}-1$. By the regularity results stated above, $w \in C^{1,2+\alpha}\left([\epsilon, T] \times \overline{D^{\prime}}\right)$ for every open set $D^{\prime} \subset \subset D$ and $0<\epsilon<T$. Differentiating $w$ with respect to time, for $(t, x) \in(0, T) \times D$ we have

$$
\begin{aligned}
\partial_{t} w & =2 \sum_{i=1}^{n} \partial_{i} v \partial_{t} \partial_{i} v \\
& =2 \sum_{i=1}^{n} \partial_{i} v \partial_{i}\left(f\left(v, \nabla^{2} v\right)-u(t, x-v \nabla v) \cdot \nabla v\right)
\end{aligned}
$$




$$
\begin{aligned}
= & 2 \sum_{i, j, k=1}^{n} \partial_{i} v f_{q_{j k}}\left(v, \nabla^{2} v\right) \partial_{i j k} v+2 \sum_{i=1}^{n} \partial_{i} v f_{s}\left(v, \nabla^{2} v\right) \partial_{i} v \\
& -2 \sum_{i=1}^{n} \partial_{i} v \sum_{j, k=1}^{n} \partial_{i}\left(x_{j}-v \partial_{j} v\right) \partial_{j} u_{k}(t, x-v \nabla v) \partial_{k} v-2 \sum_{i, k=1}^{n} \partial_{i} v \partial_{i k} v u_{k}(t, x-v \nabla v) \\
= & \sum_{i, j=1}^{n} f_{q_{i j}}\left(v, \nabla^{2} v\right) \partial_{i j} w-2 \sum_{i, j, k=1}^{n} f_{q_{i j}}\left(v, \nabla^{2} v\right) \partial_{i k} v \partial_{k j} v+2 f_{s}\left(v, \nabla^{2} v\right)\left|\partial_{x} v\right|^{2} \\
& -2 \sum_{i, j, k=1}^{n} \partial_{i} v \partial_{k} v\left(\delta_{i j}-\partial_{i} v \partial_{j} v-v \partial_{i j} v\right) \partial_{j} u_{k}(t, x-v \nabla v)-\sum_{i=1}^{n} u_{i}(t, x-v \nabla v) \partial_{i} w .
\end{aligned}
$$

Now,

and

$$
\sum_{i, j, k=1}^{n} f_{q_{i j}}\left(v, \nabla^{2} v\right) \partial_{i k} v \partial_{k j} v=\sum_{i=1}^{n} \frac{\left(\lambda_{i}\left(\nabla^{2} v\right)\right)^{2}}{\left(1-\lambda_{i}\left(\nabla^{2} v\right) v\right)^{2}}=f_{s}\left(v, \nabla^{2} v\right)
$$

$$
\begin{aligned}
\sum_{i, j, k=1}^{n} \partial_{i} v \partial_{k} v \partial_{i} v \partial_{j} v \partial_{j} u_{k}(t, x-v \nabla v) & =\left|\partial_{x} v\right|^{2} \sum_{j, k=1}^{n} \partial_{j} v \partial_{k} v \partial_{j} u_{k}(t, x-v \nabla v) \\
& =(w+1) \sum_{j, k=1}^{n} \partial_{j} v \partial_{k} v \partial_{j} u_{k}(t, x-v \nabla v), \\
v \sum_{i, j, k=1}^{n} \partial_{i} v \partial_{k} v \partial_{i j} v \partial_{j} u_{k}(t, x-v \nabla v) & =\frac{1}{2} v \sum_{j=1}^{n} \partial_{j} w \sum_{k=1}^{n} \partial_{k} v \partial_{j} u_{k}(t, x-v \nabla v)
\end{aligned}
$$

Thus

$\partial_{t} w=\sum_{i, j=1}^{n} f_{q_{i j}}\left(v, \nabla^{2} v\right) \partial_{i j} w+\sum_{i=1}^{n}\left\{u_{i}(t, x-v \nabla v)+l_{1, i}(t, x)\right\} \partial_{i} w+2\left\{f_{s}\left(v, \nabla^{2} v\right)+l_{2}(t, x)\right\} w$,

where

$$
l_{1, i}(t, x):=v \sum_{k=1}^{n} \partial_{k} v \partial_{i} u_{k}(t, x-v \nabla v), \quad l_{2}(t, x):=\sum_{j, k=1}^{n} \partial_{j} v \partial_{k} v \partial_{j} u_{k}(t, x-v \nabla v) .
$$

Since $w$ vanishes on the parabolic boundary of $[0, T] \times \bar{D}$, we have $w \equiv 0$ and $\left|\partial_{x} v\right| \equiv 1$. This implies that each $\Gamma_{t}, t>0$, is a hypersurface of class $C^{3+\alpha}$ and $v(t, x)=\nabla v(t, x)$ is a unit normal vector of $\Gamma_{t}$. In order to see that the family $\left\{\Gamma_{t}\right\}_{0 \leqslant t \leqslant T}$ is an evolving hypersurface satisfying the convected mean curvature equation (3.1), we consider the ODE

$$
\left\{\begin{array}{l}
\frac{\mathrm{d} x}{\mathrm{~d} t}=-f\left(v(t, x), \nabla^{2} v(t, x)\right) \nabla v(t, x)+u(t, x-v \nabla v(t, x)), \\
x\left(t_{0}\right)=x_{0}, \quad x_{0} \in \Gamma_{t_{0}}
\end{array}\right.
$$

for $0 \leqslant t_{0} \leqslant T$. From the regularity conditions on $v$ and $u$, the Lipschitz norm with respect to $x$ of the right hand side of the above equation has a singularity near time zero. Nevertheless, we can check that the problem is uniquely solvable and the solution $x(t)$ is at least in $C^{1}([0, T])$. Moreover, since $\frac{d}{d t} v(t, x(t))=0$ and $v\left(t_{0}, x\left(t_{0}\right)\right)=0$, we have $v(t, x(t)) \equiv 0$. Hence, $x(t) \in \Gamma_{t}$. This completes the proof. 
Let $u(t, x)$ and $\tilde{u}(t, x)$ be two functions in $\mathcal{U}_{M}$. Let $v, \tilde{v} \in C^{1,2+\alpha}([0, T] \times \bar{D})$ be the solutions of the equation (3.6) with initial data $v(0, x)=\tilde{v}(0, x)=d_{0}(x)$ and with convection terms $u$, $\tilde{u}$, respectively. We remark that for fixed $M>0$ and $d_{0}$, the above $T$ can be taken uniformly in $u \in \mathcal{U}_{M}$.

Proposition 3.3 Fix $M>0$. Let $\alpha \in(0,1)$. Assume that $\Omega_{0}$ is a bounded domain with uniformly $C^{2+\alpha}$ boundary and let $d_{0}$ be the signed distance function from $\Gamma_{0}=\partial \Omega_{0}$. Let $u, \tilde{u}$, $v, \tilde{v}$ be the functions defined above. Then

$$
\|v-\tilde{v}\|_{C^{1,2+\alpha}([0, T] \times \bar{D})} \leqslant C\|u-\tilde{u}\|_{C^{0, \alpha}\left([0, T] \times \mathbb{R}^{n}\right)},
$$

where $C$ depends only on $n, \alpha, \iota, M, \sigma_{2}, K_{f}, K_{\alpha}$, and $\left\|d_{0}\right\|_{C^{2+\alpha}}$.

Proof. Let $\mathcal{A}$ and $\mathcal{B}$ be the operators defined in the proof of Proposition 3.1. Then $w=v-\tilde{v}$ is a solution of the equation

$$
\left\{\begin{aligned}
\begin{array}{rl}
\partial_{t} w= & \mathcal{A} w+f\left(v, \nabla^{2} v\right)-f\left(\tilde{v}, \nabla^{2} \tilde{v}\right)-\mathcal{A} w \\
& \quad-u(t, x-v \nabla v) \cdot \nabla v+\tilde{u}(t, x-\tilde{v} \nabla \tilde{v}) \cdot \nabla \tilde{v}, \quad 0 \leqslant t \leqslant T, x \in \bar{D} \\
\mathcal{B} w= & -g(\nabla v)+g(\nabla \tilde{v})+\mathcal{B} w, \quad 0 \leqslant t \leqslant T, x \in \partial D \\
w(0, x)=0, \quad x \in \bar{D}
\end{array}
\end{aligned}\right.
$$

So the function $w$ satisfies

$$
\|w\|_{C^{1,2+\alpha}} \leqslant C\left(\left\|\bar{h}_{1}\right\|_{C^{0, \alpha}}+\left\|\bar{h}_{2}\right\|_{C^{0, \alpha}}+\left\|\bar{h}_{3}\right\|_{\left.C^{(1+\alpha) / 2,1+\alpha}\right)},\right.
$$

where

$$
\begin{aligned}
& \bar{h}_{1}(t, x):=f\left(v, \nabla^{2} v\right)-f\left(\tilde{v}, \nabla^{2} \tilde{v}\right)-\mathcal{A} w, \\
& \bar{h}_{2}(t, x):=u(t, x-v \nabla v) \cdot \nabla v-\tilde{u}(t, x-\tilde{v} \nabla \tilde{v}) \cdot \nabla \tilde{v}, \\
& \bar{h}_{3}(t, x):=-g(\nabla v)+g(\nabla \tilde{v})+\mathcal{B} w .
\end{aligned}
$$

The estimates for $\left\|\bar{h}_{1}\right\|_{C^{0, \alpha}}$ and $\left\|\bar{h}_{3}\right\|_{C^{0, \alpha}}$ are the same as the ones for $\left\|h_{1}\right\|_{C^{0, \alpha}}$ and $\left\|h_{3}\right\|_{C^{0, \alpha}}$ in the proof of Proposition 3.1 , respectively. So we have

$$
\left\|\bar{h}_{1}\right\|_{C^{0, \alpha}}+\left\|\bar{h}_{3}\right\|_{C^{0, \alpha}} \leqslant C T^{\alpha / 2}\|v-\tilde{v}\|_{C^{1,2+\alpha}} .
$$

We decompose $\bar{h}_{2}$ as

$$
\begin{aligned}
\bar{h}_{2}(t, x) & =u(t, x-v \nabla v) \cdot \nabla v-u(t, x-\tilde{v} \nabla \tilde{v}) \cdot \nabla \tilde{v}+(u(t, x-\tilde{v} \nabla \tilde{v})-\tilde{u}(t, x-\tilde{v} \nabla \tilde{v})) \cdot \nabla \tilde{v} \\
& =: \bar{h}_{2,1}(t, x)+\bar{h}_{2,2}(t, x) .
\end{aligned}
$$

The estimate for $\bar{h}_{2,1}$ is the same as the one for $h_{2}$ in the proof of Proposition 3.1, so

$$
\left\|\bar{h}_{2,1}\right\|_{C^{0, \alpha}} \leqslant C T^{\alpha / 2}\|v-\tilde{v}\|_{C^{1,2+\alpha}} .
$$

The estimate for $\bar{h}_{2,2}$ is also easy:

$$
\left\|\bar{h}_{2,2}\right\|_{C^{0, \alpha}} \leqslant C\|u-\tilde{u}\|_{C^{0, \alpha}\left([0, T] \times \mathbb{R}^{n}\right)} .
$$

Thus for sufficiently small $T^{\prime} \leqslant T$, we obtain

$$
\|v-\tilde{v}\|_{C^{1,2+\alpha}\left(\left[0, T^{\prime}\right] \times \bar{D}\right)} \leqslant C\|u-\tilde{u}\|_{C^{0, \alpha}\left(\left[0, T^{\prime}\right] \times \mathbb{R}^{n}\right)} .
$$

Repeating this argument, we have the desired estimate. The dependence of the constant is obvious. Now the proof is complete. 


\section{Mild solutions of the Stokes equations}

In this section, we construct mild solutions of the Stokes equations with the layer potential. Now we define a mild solution as a solution of the integral equation associated with the Stokes equations. Let $\mathcal{S}^{\prime}\left(\mathbb{R}^{n}\right)$ be the class of tempered distributions.

DEFINITION 4.1 Let $h$ be a bounded continuous function in $(0, T] \times \mathbb{R}^{n}$. Let $\left\{\Gamma_{t}\right\}_{0 \leqslant t \leqslant T}$ be a family of hypersurfaces with suitable regularity. The function $u$ is called a mild solution of the Stokes equation with the layer potential term $h \mathcal{H}_{\left\llcorner\Gamma_{t}\right.}^{n-1}$ if there exists $u_{0} \in \mathcal{S}^{\prime}\left(\mathbb{R}^{n}\right)$ with $\nabla \cdot u_{0}=0$ such that

$$
u(t)=e^{t \Delta} u_{0}+\int_{0}^{t} e^{(t-s) \Delta} \mathbf{P} h \mathcal{H}_{\left\llcorner\Gamma_{s}\right.}^{n-1} \mathrm{~d} s .
$$

Here, $e^{t \Delta}$ is the heat semigroup, and $\mathbf{P}$ is the Helmholtz projection.

Since both $e^{t \Delta}$ and $\mathbf{P}$ are convolution operators, we can regard $e^{t \Delta} \mathbf{P}$ as one convolution operator. More precisely, for $a \in\left(C_{0}\left(\mathbb{R}^{n}\right)\right)^{n}$, the $i$-th component of the convolution $e^{t \Delta} \mathbf{P} a$ is expressed as

$$
\left(e^{t \Delta} \mathbf{P} a\right)^{(i)}=\sum_{j=1}^{n}\left(\frac{1}{t^{n / 2}} L_{i, j}\left(\frac{\cdot}{\sqrt{t}}\right)+G_{t}(\cdot) \delta_{i j}\right) * a_{j} .
$$

Here, $G_{t}(x)$ is the Gauss kernel

$$
G_{t}(x):=\frac{1}{(4 \pi t)^{n / 2}} \exp \left(-\frac{|x|^{2}}{4 t}\right)
$$

and

$$
L_{j, k}:=-\mathcal{F}^{-1}\left(\frac{\xi_{j} \xi_{k}}{|\xi|^{2}} \exp \left(-|\xi|^{2}\right)\right),
$$

where $\mathcal{F}^{-1}$ is the inverse Fourier transform,

$$
\mathcal{F}^{-1} g(x):=(2 \pi)^{-n / 2} \int_{\mathbb{R}^{n}} g(\xi) e^{i x \cdot \xi} \mathrm{d} \xi
$$

The pointwise estimates of the kernel function

$$
K_{i, j}(t, x):=\frac{1}{t^{n / 2}} L_{i, j}\left(\frac{\cdot}{\sqrt{t}}\right)+G_{t}(\cdot) \delta_{i j}
$$

are given as follows.

LEMMA 4.1 Let $K_{i, j}(t, x)$ be as defined above. Let $l=\left(l_{1}, \ldots, l_{n}\right)$ be a multi-index. Then

$$
\left|\partial_{x}^{l} K_{i, j}(t, x)\right| \leqslant \frac{C}{t^{(n+|l|) / 2}}\left(1+\frac{|x|}{\sqrt{t}}\right)^{-(n+|l|)},
$$

where $C$ depends only on $n$ and $l$.

Proof. These pointwise estimates were originally obtained by C. W. Oseen [18]. See also P. G. Lemarié-Rieusset [11], and Y. Shibata and S. Shimizu [22]. A simple proof was also obtained by the author and Y. Terasawa [14].

For the heat semigroup $e^{t \Delta}$, we have the following estimates. 
Lemma 4.2 Let $0<\alpha<1$. Let $b(t, x):=e^{t \Delta} a$. Then

$$
\begin{aligned}
\|b\|_{C\left([0, T] \times \mathbb{R}^{n}\right)} & \leqslant C\|a\|_{C\left(\mathbb{R}^{n}\right)}, \\
{[b]_{C^{\alpha / 2, \alpha}\left([0, T] \times \mathbb{R}^{n}\right)} } & \leqslant C[a]_{C^{\alpha}\left(\mathbb{R}^{n}\right)}, \\
{[b]_{C^{\alpha / 2, \alpha}\left([t, T] \times \mathbb{R}^{n}\right)} } & \leqslant \frac{C}{t^{\alpha / 2}}\|a\|_{C\left(\mathbb{R}^{n}\right)} .
\end{aligned}
$$

Proof. These estimates are well known, so we omit the proof. See [13, Chapter 5.1.1], for example.

\subsection{Estimates for the layer potential}

In this section, we shall estimate the term

$$
F(t, x):=\int_{0}^{t} e^{(t-s) \Delta} \mathbf{P} h \mathcal{H}_{\left\llcorner\Gamma_{s}\right.}^{n-1} \mathrm{~d} s,
$$

which reflects the boundary condition on $\Gamma_{t}$ when $h=H \nu$. First, we define the class of evolving hypersurfaces we shall deal with. Let $\Gamma_{0}$ be the boundary of a smooth bounded domain $\Omega_{0}$. Let $d_{0}$ be the signed distance function of $\Gamma_{0}$,

$$
d_{0}(x)= \begin{cases}\operatorname{dist}\left(x, \Gamma_{0}\right), & x \in \mathbb{R}^{n} \backslash \bar{\Omega}_{0}, \\ -\operatorname{dist}\left(x, \Gamma_{0}\right), & x \in \Omega_{0} .\end{cases}
$$

We set

$$
D:=\left\{x \in \mathbb{R}^{n} ;-\delta_{0}<d_{0}(x)<\delta_{0}\right\}
$$

for sufficiently small $\delta_{0}$; see Section 3. We assume that $\Gamma_{0}$ is uniformly $C^{2+\alpha}$, that is, $d_{0} \in C^{2+\alpha}(\bar{D})$. Since $d_{0}$ is a distance function, we have $\left|\partial_{x} d_{0}(x)\right| \equiv 1$ on $x \in \bar{D}$, which implies that

$$
\min _{x \in \bar{D}} \max _{1 \leqslant i \leqslant n}\left|\partial_{i} d_{0}(x)\right| \geqslant 1 / n \text {. }
$$

We set

$$
r:=\min \left\{1 / n, \delta_{0}\right\}>0 .
$$

Note that $r$ depends only on $n$ and $\Gamma_{0}$.

Definition 4.2 Let $R \geqslant 1$ be a given number and $\alpha \in(0,1)$. We define $\mathcal{S}\left(\alpha, R, T, d_{0}\right)$ as the set of families $\left\{\Gamma_{t}\right\}_{0 \leqslant t \leqslant T}$ of hypersurfaces such that each $\Gamma_{t}$ is the boundary of a bounded domain $\Omega_{t} \subset \mathbb{R}^{n}$ and is represented as

$$
\Gamma_{t}=\{x \in D ; v(t, x)=0\}
$$

by the signed distance function $v \in C^{1,2+\alpha}([0, T] \times \bar{D})$ satisfying $\|v\|_{C^{1,2+\alpha}([0, T] \times \bar{D})} \leqslant R$ and $v(0, x)=d_{0}(x)$.

By the implicit function theorem, we can derive the properties of the local coordinate transforms of $\left\{\Gamma_{t}\right\}_{0 \leqslant t \leqslant T}$ in $\mathcal{S}\left(\alpha, R, T, d_{0}\right)$; see Section 6.2 .

Let $R \geqslant 1$, and let $T_{0}$ be a positive number given by Proposition 6.1 depending only on $R$ and $d_{0}$. If $T \leqslant T_{0}$ and $\left\{\Gamma_{t}\right\}_{0 \leqslant t \leqslant T}$ is an evolving hypersurface belonging to $\mathcal{S}\left(\alpha, R, T, d_{0}\right)$, then the following statements hold from Proposition 6.1 
(a) There exists a family of open sets $\left\{U_{k}\right\}_{k=1}^{m}, U_{k} \subset \bar{D}$, and a family of functions $\left\{\varphi_{k}(t, x)\right\}_{k=1}^{m}$ with $\varphi_{k}(t, x) \in C^{1,2+\alpha}\left([0, T] \times \bar{U}_{k}\right)$ satisfying the following conditions.

(b) For each $t \in[0, T]$, there exists an open set $U_{j}(t) \subset U_{j}$ such that:

(b-1) The functions $\varphi_{k}(t, \cdot): \overline{U_{k}(t)} \rightarrow \bar{B}$, where $B:=\left\{y \in \mathbb{R}^{n} ;|y|<1\right\}$, are $C^{2}$-diffeomorphisms.

(b-2) For each $t \in[0, T]$,

$$
\varphi_{k}\left(t,\left\{\overline{U_{k}(t)} \cap \Omega_{t}\right\}\right)=\left\{y \in \bar{B} ; y_{n}>0\right\}
$$

and

$$
\varphi_{k}\left(t,\left\{\overline{U_{k}(t)} \cap \Gamma_{t}\right\}\right)=\overline{B^{\prime}}, \quad B^{\prime}:=\left\{y \in B ; y_{n}=0\right\} .
$$

(b-3) For some $\rho>0$, there exist families of open balls $\left\{O_{k}\right\}_{k=1}^{m}$ and $\left\{\hat{O}_{k}\right\}_{k=1}^{m}$ such that

$$
\begin{aligned}
\hat{O}_{k} & \subset \subset O_{k}, \\
O_{k} & \subset \subset \bigcap_{0 \leqslant t \leqslant T} \varphi_{k}^{-1}(t, B), \quad 1 \leqslant k \leqslant m, \\
\bigcup_{0 \leqslant t \leqslant T}\left(\Gamma_{t}\right)_{\rho} & \subset \subset \bigcup_{k=1}^{m} \hat{O}_{k},
\end{aligned}
$$

where $(A)_{\rho}:=\left\{x \in \mathbb{R}^{n} ; \operatorname{dist}(x, A)<\rho\right\}$.

We can choose $\rho,\left\{U_{k}\right\}_{k=1}^{m},\left\{O_{k}\right\}_{k=1}^{m}$, and $\left\{\hat{O}_{k}\right\}_{k=1}^{m}$ not depending on the evolving hypersurface belonging to $\bigcup_{0<T \leqslant T_{0}} \mathcal{S}\left(\alpha, R, T, d_{0}\right)$. In particular, we can take $O_{k}=\left\{\left|x-\bar{x}_{k}\right|<4 \rho\right\}$ and $\hat{O}_{k}=$ $\left\{\left|x-\bar{x}_{k}\right|<3 \rho\right\}$ for some $\bar{x}_{k} \in \Gamma_{0}$. Moreover, if $\max _{1 \leqslant i \leqslant n}\left|\partial_{i} d_{0}\left(\bar{x}_{k}\right)\right|=\left|\partial_{i_{0}} d_{0}\left(\bar{x}_{k}\right)\right|$, the local coordinate transforms $\varphi_{k}=\left(\varphi_{k}^{(1)}, \ldots, \varphi_{k}^{(n)}\right), \psi_{k}=\left(\psi_{k}^{(1)}, \ldots, \psi_{k}^{(n)}\right)$ can be taken as

$$
\begin{aligned}
\varphi_{k}(t, x) & =\Pi \hat{\varphi}_{k}(t, x), \\
\psi_{k}(t, y) & =\hat{\psi}_{k}\left(t, \Pi^{-1} y\right),
\end{aligned}
$$

where $\Pi$ is an orthogonal matrix such that $\Pi\left(x^{(1)}, \ldots, x^{\left(i_{0}\right)}, \ldots, x^{(n)}\right)=\left(x^{(1)}, \ldots, x^{(n)}\right.$, $\left.\ldots, x^{\left(i_{0}\right)}\right)$ for all $x=\left(x^{(1)}, \ldots, x^{(n)}\right)$ and

$$
\begin{aligned}
\hat{\varphi}_{k}^{(i)}(t, x) & =\frac{64 R^{2}}{r^{2}}\left(x^{(i)}-\bar{x}_{k}^{(i)}\right), \quad i \neq i_{0}, \\
\hat{\varphi}_{k}^{\left(i_{0}\right)}(t, x) & =\frac{64 R^{2}}{r^{2}}\left(x^{\left(i_{0}\right)}-g_{k}\left(t, x^{(1)}, \stackrel{i_{0}}{\cdot}, x^{(n)}\right)\right), \\
\hat{\psi}_{k}^{(i)}(t, y) & =\frac{r^{2}}{64 R^{2}} y^{(i)}+\bar{x}_{k}^{(i)}, \quad i \neq i_{0}, \\
\hat{\psi}_{k}^{\left(i_{0}\right)}(t, y) & =\frac{r^{2}}{64 R^{2}} y^{\left(i_{0}\right)}+g_{k}\left(t, \frac{r^{2}}{64 R^{2}} y^{(1)}+\bar{x}_{k}^{(1)}, \stackrel{i_{0}}{\cdot}, \frac{r^{2}}{64 R^{2}} y^{(n)}+\bar{x}_{k}^{(n)}\right) .
\end{aligned}
$$

Here, $g_{k}$ is the function constructed in Proposition 6.1. For these local coordinate transforms, we have the following lemma. Let $y=\left(y^{\prime}, y^{(n)}\right)$. 
LEMma 4.3 Let $\left\{\Gamma_{t}\right\}_{0 \leqslant t \leqslant T}$ be an evolving hypersurface belonging to $\mathcal{S}\left(\alpha, R, T, d_{0}\right)$. Let $\left\{\varphi_{k}\right\}_{k=1}^{m},\left\{\psi_{k}\right\}_{k=1}^{m}$ be the local coordinate transforms of $\left\{\Gamma_{t}\right\}_{0 \leqslant t \leqslant T}$ above. Then

$$
\begin{aligned}
& \left\|\varphi_{k}\right\|_{C^{1,2+\alpha}\left([0, T] \times \bar{U}_{k}\right)},\left\|\psi_{k}\right\|_{C^{1,2+\alpha}([0, T] \times \bar{B})} \leqslant C\left(1+\|v\|_{C^{1,2+\alpha}([0, T] \times \bar{D})}\right), \\
& \left|\psi_{k}\left(t, y_{1}\right)-\psi_{k}\left(s, y_{2}\right)\right| \geqslant C\left|y_{1}^{\prime}-y_{2}^{\prime}\right| \quad \text { for } 0 \leqslant s \leqslant t \leqslant T,
\end{aligned}
$$

where $C$ depends only on $n, r$, and $R$.

Moreover, let $\left\{\tilde{\Gamma}_{t}\right\}_{0 \leqslant t \leqslant T}$ be another evolving hypersurface belonging to $\mathcal{S}\left(\alpha, R, T, d_{0}\right)$ and let $\left\{\tilde{\varphi}_{k}\right\}_{k=1}^{m},\left\{\tilde{\psi}_{k}\right\}_{k=1}^{m}$ be the local coordinate transforms of $\left\{\tilde{\Gamma}_{t}\right\}_{0 \leqslant t \leqslant T}$ given by $4.16-4.21$. Assume that $l_{1}, l_{2} \in \mathbb{N},\left\{t_{i}\right\}_{i=1}^{l_{1}+l_{2}} \subset[0, T],\left\{y_{i}\right\}_{i=1}^{l_{1}+l_{2}} \subset B$, and $\left\{\tau_{i}\right\}_{i=1}^{l_{1}+l_{2}} \subset \mathbb{R}$ with $\sum_{i=1}^{l_{1}+l_{2}} \tau_{i}=0$. Then

$$
\begin{gathered}
\left.\left\|\varphi_{k}-\tilde{\varphi}_{k}\right\|_{C^{1,2+\alpha}\left([0, T] \times \bar{U}_{k}\right)}+\left\|\psi_{k}-\tilde{\psi}_{k}\right\|_{C^{1,2+\alpha}([0, T] \times \bar{B})} \leqslant C\|v-\tilde{v}\|_{C^{1,2+\alpha}([0, T] \times \bar{D})}\right), \\
\left|\sum_{i=1}^{l_{1}} \tau_{i} \psi_{k}\left(t_{i}, y_{i}\right)+\sum_{i=l_{1}+1}^{l_{1}+l_{2}} \tau_{i} \tilde{\psi}_{k}\left(t_{i}, y_{i}\right)\right| \geqslant C\left|\sum_{i=1}^{l_{1}+l_{2}} \tau_{i} y_{i}^{\prime}\right|,
\end{gathered}
$$

where $C$ depends only on $n, r$, and $R$.

Proof. The estimates (4.22) and (4.24) are obvious by Proposition 6.1 The estimate (4.23) follows from 4.25 . So we shall only prove 4.25 . From the definition of $\psi_{k}$ and $\tilde{\psi}_{k}$, we have

$$
\begin{aligned}
\left|\sum_{i=1}^{l_{1}} \tau_{i} \psi_{k}\left(t_{i}, y_{i}\right)+\sum_{i=l_{1}+1}^{l_{1}+l_{2}} \tau_{i} \tilde{\psi}_{k}\left(t_{i}, y_{i}\right)\right| & \geqslant\left\{\sum_{j \neq i_{0}}\left(\sum_{i=1}^{l_{1}} \tau_{i} \psi_{k}^{(j)}\left(t_{i}, y_{i}\right)+\sum_{i=l_{1}+1}^{l_{1}+l_{2}} \tau_{i} \tilde{\psi}_{k}^{(i)}\left(t_{i}, y_{i}\right)\right)^{2}\right\}^{1 / 2} \\
& =\left\{\sum_{j \neq i_{0}}\left[\sum_{i=1}^{l_{1}+l_{2}} \tau_{i}\left(\frac{r^{2}}{64 R^{2}}\left(\Pi^{-1} y_{i}\right)^{(j)}+\bar{x}_{k}^{(j)}\right)\right]^{2}\right\}^{1 / 2} \\
& =\left\{\sum_{j \neq i_{0}}\left[\sum_{i=1}^{l_{1}+l_{2}} \frac{r^{2}}{64 R^{2}} \tau_{i}\left(\Pi^{-1} y_{i}\right)^{(j)}\right]^{2}\right\}^{1 / 2} \\
& =\frac{r^{2}}{64 R^{2}}\left\{\sum_{j=1}^{n-1}\left[\sum_{i=1}^{l_{1}+l_{2}} \tau_{i} y_{i}^{(j)}\right]^{2}\right\}^{1 / 2}=\frac{r^{2}}{64 R^{2}}\left|\sum_{i=1}^{l_{1}+l_{2}} \tau_{i} y_{i}^{\prime}\right| .
\end{aligned}
$$

This completes the proof.

Let $K_{i, j}(t, x):=t^{-n / 2} L_{i, j}(x / \sqrt{t})+G_{t}(x) \delta_{i j}$ be the $(i, j)$-component of the kernel of the matrix convolution operator $e^{t \Delta} \mathbf{P}$. Combining the above estimate 4.25 with the pointwise estimate [4.3), we have

$$
\begin{aligned}
\left|\left(\partial_{x}^{\theta} K_{i, j}\right)\left(t, \sum_{i=1}^{l_{1}} \tau_{i} \psi_{k}\left(t_{i}, y_{i}\right)+\sum_{i=l_{1}+1}^{l_{1}+l_{2}} \tau_{i} \tilde{\psi}_{k}\left(t_{i}, y_{i}\right)\right)\right| & \\
& \leqslant C t^{-(n+|\theta|) / 2}\left(1+C \frac{\left|\sum_{i=1}^{l_{1}+l_{2}} \tau_{i} y_{i}^{\prime}\right|}{\sqrt{t}}\right)^{-n-|\theta|}
\end{aligned}
$$

for any multi-index $\theta=\left(\theta_{1}, \ldots, \theta_{n}\right)$. 
Let $\left\{a_{k}\right\}_{k=1}^{m}$ be a partition of unity for $\bigcup_{k=1}^{m} \hat{O}_{k}$ subordinate to $\left\{O_{k}\right\}_{k=1}^{m}$, i.e.: for every $k$, $a_{k}(x)$ is smooth and $0 \leqslant a_{k} \leqslant 1$; for every $k$, supp $a_{k} \subset O_{k}$; for every $x \in \bigcup_{k=1}^{m} \hat{O}_{k}$, we have $\sum_{k=1}^{m} a_{k}(x)=1$. Note that we can take $\left\{a_{k}\right\}_{k=1}^{m}$ not depending on the evolving hypersurface belonging to $\bigcup_{0<T \leqslant T_{0}} \mathcal{S}\left(\alpha, R, T, d_{0}\right)$. We set

$$
\delta_{1}:=\min _{1 \leqslant k \leqslant m} \operatorname{dist}\left(O_{k}^{c}, \operatorname{supp} a_{k}\right)>0
$$

We may assume that $\delta_{1} \geqslant \frac{7}{2} \rho$. The next proposition plays an essential role in this paper.

Proposition 4.1 Let $p \in(1, \infty]$ and $\alpha, \beta \in(0,1)$. Assume that $R \geqslant 1$ is a given number and $\Gamma_{0}$ is a given $C^{2+\alpha}$ hypersurface. Let $d_{0}$ be the signed distance function, $T_{0}$ be a positive number in Proposition 6.1. and $r$ be the number defined by (4.11). Let $\left\{\Gamma_{t}\right\}_{0 \leqslant t \leqslant T}$ be an evolving hypersurface belonging to $\mathcal{S}\left(\alpha, R, T, d_{0}\right)$ for some $T \leqslant T_{0}$. Then the function $F(t, x)$ given by (4.7) satisfies the following estimates:

$$
\begin{aligned}
\|F\|_{C^{\beta / 2, \beta}\left([0, T] \times \mathbb{R}^{n}\right)} & \leqslant C_{1} T^{(1-\beta) / 2}\|h\|_{C([0, T] \times \bar{D})}, \\
\sup _{0 \leqslant t \leqslant T}\|F(t)\|_{L^{p}\left(\mathbb{R}^{n}\right)} & \leqslant C_{2} T^{1 / 2}\|h\|_{C([0, T] \times \bar{D})}, \\
\sup _{0 \leqslant t \leqslant T}\|F(t)\|_{C^{1+\alpha}\left(\Gamma_{t}\right)} & \leqslant C_{3}\|h\|_{C^{0, \alpha}([0, T] \times \bar{D})},
\end{aligned}
$$

where $C_{1}=C_{1}(n, \beta, r, R), C_{2}=C_{2}(n, p, r, R)$, and $C_{3}=C_{3}(n, \alpha, r, R)$.

Proof. For simplicity of notation, we write $\|h\|_{C},\|h\|_{C^{0, \alpha}}$ instead of $\|h\|_{C([0, T] \times \bar{D})}$, $\|h\|_{C^{0, \alpha}([0, T] \times \bar{D})}$, respectively. First, we shall prove the estimate $(4.28)$. Note that

$$
\begin{aligned}
& \|F(t, \cdot)\|_{C\left(\mathbb{R}^{n}\right)} \leqslant \int_{0}^{t}\left\|e^{(t-s) \Delta} \mathbf{P} h \mathcal{H}_{\left\llcorner\Gamma_{s}\right.}^{n-1}\right\|_{C\left(\mathbb{R}^{n}\right)} \mathrm{d} s \\
& {[F(t, \cdot)]_{C^{\beta}\left(\mathbb{R}^{n}\right)} \leqslant C \int_{0}^{t}(t-s)^{-\beta / 2}\left\|e^{(t-s) / 2 \Delta} \mathbf{P} h \mathcal{H}_{\left\llcorner\Gamma_{s}^{n-1}\right.}\right\|_{C\left(\mathbb{R}^{n}\right)} \mathrm{d} s,}
\end{aligned}
$$

and

$$
\begin{aligned}
|F(t, x)-F(\tau, x)| \leqslant & \int_{\tau}^{t}\left\|e^{(t-s) \Delta} \mathbf{P} h \mathcal{H}_{\left\llcorner\Gamma_{s}\right.}^{n-1}\right\|_{C\left(\mathbb{R}^{n}\right)} \mathrm{d} s \\
& +\int_{0}^{\tau}\left\|\left(e^{(t-\tau) \Delta}-I\right) e^{(\tau-s) \Delta} \mathbf{P} h \mathcal{H}_{\left\llcorner\Gamma_{s}\right.}^{n-1}\right\|_{C\left(\mathbb{R}^{n}\right)} \mathrm{d} s \\
\leqslant & \int_{\tau}^{t}\left\|e^{(t-s) \Delta} \mathbf{P} h \mathcal{H}_{\left\llcorner\Gamma_{s}\right.}^{n-1}\right\|_{C\left(\mathbb{R}^{n}\right)} \mathrm{d} s \\
& +C(t-\tau)^{\beta / 2} \int_{0}^{\tau}(\tau-s)^{-\beta / 2}\left\|e^{(\tau-s) / 2 \Delta} \mathbf{P} h \mathcal{H}_{\left\llcorner\Gamma_{s}-1\right.}\right\|_{C\left(\mathbb{R}^{n}\right)} \mathrm{d} s .
\end{aligned}
$$

Thus it suffices to estimate

$$
\left\|e^{(t-s) \Delta} \mathbf{P} h \mathcal{H}_{\left\llcorner\Gamma_{s}\right.}^{n-1}\right\|_{C\left(\mathbb{R}^{n}\right)}
$$


The $i$-th component of $e^{(t-s) \Delta} \mathbf{P} h \mathcal{H}_{\left\llcorner\Gamma_{s}\right.}^{n-1}$ is given as

$$
\begin{aligned}
\sum_{j=1}^{n} \int_{\Gamma_{s}} K_{i, j}(t-s, x-y) & h_{j}(s, y) \mathcal{H}^{n-1}(\mathrm{~d} y) \\
= & \sum_{j=1}^{n} \sum_{k=1}^{m} \int_{\Gamma_{s} \cap O_{k}} a_{k}(y) K_{i, j}(t-s, x-y) h_{j}(s, y) \mathcal{H}^{n-1}(\mathrm{~d} y) \\
= & : \sum_{j=1}^{n} \sum_{k=1}^{m} \mathcal{I}_{i, j, k}(t, s, x) .
\end{aligned}
$$

So we shall estimate $\mathcal{I}_{i, j, k}(t, s, x)$. By the area formula, we have

$$
\mathcal{I}_{i, j, k}(t, s, x)=\int_{\mathbb{R}^{n-1}} K_{i, j}\left(t-s, x-\psi_{k}\left(s, \xi^{\prime}, 0\right)\right) \Upsilon_{1}\left(s, \xi^{\prime}\right) \mathrm{d} \xi^{\prime},
$$

where

$$
\Upsilon_{1}\left(s, \xi^{\prime}\right)=a_{k}\left(\psi_{k}\left(s, \xi^{\prime}, 0\right)\right) h_{j}\left(s, \psi_{k}\left(s, \xi^{\prime}, 0\right)\right) J_{\psi_{k}}\left(s, \xi^{\prime}\right) .
$$

Here

$$
J_{\psi_{k}}\left(s, \xi^{\prime}\right)=\left.\left[\sum_{l=1}^{n}\left\{\frac{\partial\left(\psi_{k}^{(1)}, \stackrel{l}{l}, \psi_{k}^{(n)}\right)}{\partial\left(\xi^{(1)}, \ldots, \xi^{(n-1)}\right)}\right\}^{1 / 2}\right]\right|_{t=s, \xi^{(n)}=0} .
$$

Note that the integrand in the above integration is naturally extended by zero to $\mathbb{R}^{n-1}$ thanks to the inclusion $B^{\prime} \cap \operatorname{supp} a_{k}\left(\psi_{k}(s, \cdot)\right) \subset B^{\prime} \cap \varphi_{k}\left(s, O_{k}\right)$.

It is easy to obtain the estimate $\left|\Upsilon_{1}\left(s, \xi^{\prime}\right)\right| \leqslant C$ where $C$ depends only on $n, r$, and $R$. If $x \in O_{k}$, then there exists a point $\bar{\xi}(s, x) \in B$ such that $x=\psi_{k}(s, \bar{\xi})$ for each $s \in[0, T]$. Thus from 4.26 ,

$$
\begin{aligned}
\left|\int_{\mathbb{R}^{n-1}} K_{i, j}\left(t-s, \psi_{k}(s, \bar{\xi})-\psi_{k}\left(s, \xi^{\prime}, 0\right)\right) \Upsilon_{1}\left(s, \xi^{\prime}\right) \mathrm{d} \xi^{\prime}\right| & \\
\leqslant & C(t-s)^{-n / 2} \int_{\mathbb{R}^{n-1}}\left(1+C \frac{\left|\bar{\xi}^{\prime}-\xi^{\prime}\right|}{(t-s)^{1 / 2}}\right)^{-n} \mathrm{~d} \xi^{\prime}\|h\|_{C} \\
& =(t-s)^{-1 / 2} \int_{\mathbb{R}^{n-1}}\left(1+C\left|z^{\prime}\right|\right)^{-n} \mathrm{~d} z^{\prime}\|h\|_{C}=C(t-s)^{-1 / 2}\|h\|_{C} .
\end{aligned}
$$

In the second last integration, we changed the variable as $\bar{\xi}^{\prime}-\xi^{\prime}=(t-s)^{1 / 2} z^{\prime}$.

If $x \notin O_{k}$, then $|x-y| \geqslant \delta_{1}>0$ for all $y \in \operatorname{supp} a_{k}$. Hence

$$
\begin{aligned}
& \mid \int_{\mathbb{R}^{n-1}} K_{i, j}(t-s, x\left.-\psi_{k}\left(s, \xi^{\prime}, 0\right)\right) \Upsilon_{1}\left(s, \xi^{\prime}\right) \mathrm{d} \xi^{\prime} \mid \\
& \leqslant C(t-s)^{-n / 2} \int_{B^{\prime} \cap \operatorname{supp} a_{k}\left(\psi_{k}(s, \cdot)\right)}\left(1+\frac{\left|x-\psi_{k}\left(s, \xi^{\prime}, 0\right)\right|}{(t-s)^{1 / 2}}\right)^{-n} \mathrm{~d} \xi^{\prime}\|h\|_{C} \\
& \leqslant C(t-s)^{-n / 2}\left(1+\frac{\delta_{1}}{(t-s)^{1 / 2}}\right)^{-n} \int_{B^{\prime}} \mathrm{d} \xi^{\prime}\|h\|_{C} \leqslant C\|h\|_{C} .
\end{aligned}
$$

Collecting these estimates, we obtain

$$
\left\|\mathcal{I}_{i, j, k}(t, s, \cdot)\right\|_{C\left(\mathbb{R}^{n}\right)} \leqslant C(t-s)^{-1 / 2}\|h\|_{C}
$$


where $C$ depends only on $n, r$ and $R$ (note that we can take $\delta_{1}$ depending only on $r$ and $R$ ). The estimate 4.28 immediately follows from the above.

Next we shall show the estimate (4.29). Note again that in 4.13)-(4.15), we can take $O_{k}=$ $\left\{\left|x-\bar{x}_{k}\right|<4 \rho\right\}$ for some $\bar{x}_{k} \in \Gamma_{0}$ from Proposition 6.1 Set $D_{k}:=\left\{\left|x-\bar{x}_{k}\right|<8 \rho\right\}$. By the estimate 4.33, we have

$$
\left\|\mathcal{I}_{i, j, k}(t, s, \cdot)\right\|_{L^{p}\left(D_{k}\right)} \leqslant\left|D_{k}\right|^{1 / p}\left\|\mathcal{I}_{i, j, k}(t, s, \cdot)\right\|_{L^{\infty}\left(D_{k}\right)} \leqslant C(t-s)^{-1 / 2}\|h\|_{C} .
$$

For $x \notin D_{k}$, we see that for all $y \in O_{k}$,

$$
|x-y| \geqslant \frac{1}{4}\left|x-\bar{x}_{k}\right|+2 \rho .
$$

Thus from the estimate 4.26 ,

$$
\begin{aligned}
\left\|\mathcal{I}_{i, j, k}(t, s, \cdot)\right\|_{L^{p}\left(\mathbb{R}^{n} \backslash D_{k}\right)} & \leqslant C(t-s)^{-n / 2}\left\|\int_{B^{\prime}}\left(1+\frac{\left|x-\psi_{k}\left(s, \xi^{\prime}, 0\right)\right|}{(t-s)^{1 / 2}}\right)^{-n} \mathrm{~d} \xi^{\prime}\right\|_{L_{x}^{p}\left(\mathbb{R}^{n} \backslash D_{k}\right)}\|h\|_{C} \\
& \leqslant C(t-s)^{-n / 2}\left\|\int_{\mathbb{B}^{\prime}}\left(1+\frac{2 \rho}{(t-s)^{1 / 2}}+\frac{\left|x-\bar{x}_{k}\right|}{4(t-s)^{1 / 2}}\right)^{-n} \mathrm{~d} \xi^{\prime}\right\|_{L_{x}^{p}\left(\mathbb{R}^{n}\right)}\|h\|_{C} \\
& \leqslant C\|h\|_{C} .
\end{aligned}
$$

Combining these, we obtain the estimate 4.29.

Finally, we shall prove the estimate 4.30 . Since

$$
\|F(t)\|_{C^{1+\alpha}\left(\Gamma_{t}\right)}:=\sup _{1 \leqslant h \leqslant m} \| F\left(t, \psi_{h}(t, \cdot, 0) \|_{C^{1+\alpha}\left(\overline{B^{\prime}}\right)},\right.
$$

we shall estimate $F\left(t, \psi_{h}\left(t, \zeta^{\prime}, 0\right)\right)$, or, by 4.31,

$$
\mathcal{J}\left(t, \zeta^{\prime}\right):=\int_{0}^{t} \mathcal{I}_{i, j, k, h}\left(t, s, \zeta^{\prime}\right) \mathrm{d} s,
$$

where $\mathcal{I}_{i, j, k, h}\left(t, s, \zeta^{\prime}\right):=\mathcal{I}_{i, j, k}\left(t, s, \psi_{h}\left(t, \zeta^{\prime}, 0\right)\right)$.

Let $\eta \in(0,1)$. In order to obtain the optimal regularity, we divide $\mathcal{J}\left(t, \zeta^{\prime}\right)$ into $\mathcal{J}_{1}\left(t, \zeta^{\prime}\right)$ and $\mathcal{J}_{2}\left(t, \zeta^{\prime}\right)$ where

$$
\begin{aligned}
& \mathcal{J}_{1}\left(t, \zeta^{\prime}\right):=\int_{(1-\eta) t}^{t} \mathcal{I}_{i, j, k, h}\left(t, s, \zeta^{\prime}\right) \mathrm{d} s \\
& \mathcal{J}_{2}\left(t, \zeta^{\prime}\right):=\int_{0}^{(1-\eta) t} \mathcal{I}_{i, j, k, h}\left(t, s, \zeta^{\prime}\right) \mathrm{d} s .
\end{aligned}
$$

We shall show that

$$
\begin{aligned}
\|\mathcal{J}(t, \cdot)\|_{C^{\alpha}\left(B^{\prime}\right)} & \leqslant C t^{1 / 2}\|h\|_{C^{0, \alpha}}, \\
\left\|\mathcal{J}_{1}(t, \cdot)\right\|_{C^{\alpha}\left(B^{\prime}\right)} & \leqslant C(\eta t)^{1 / 2}\|h\|_{C^{0, \alpha}}, \\
\left\|\mathcal{J}_{2}(t, \cdot)\right\|_{C^{2+\alpha}\left(B^{\prime}\right)} & \leqslant C(\eta t)^{-1 / 2}\|h\|_{C^{0, \alpha}} .
\end{aligned}
$$

First, we set

$$
\begin{aligned}
& B_{1}^{\prime}:=B^{\prime} \cap \varphi_{h}\left(t, O_{k} \cap U_{h}(t)\right), \\
& B_{2}^{\prime}:=B^{\prime} \cap \varphi_{h}\left(t,\left\{x \in U_{h}(t) ; \operatorname{dist}\left(x, \operatorname{supp} a_{k}\right)>\delta_{1} / 2\right\}\right) \neq \emptyset .
\end{aligned}
$$


Then both $B_{1}^{\prime}$ and $B_{2}^{\prime}$ are relatively open in $\mathbb{R}^{n-1}$, so from now on we regard $B^{\prime}, B_{1}^{\prime}$, and $B_{2}^{\prime}$ as open sets in $\mathbb{R}^{n-1}$. For all $y^{\prime} \in B^{\prime}$ there exists a unique $x \in U_{h}(t)$ such that $y=\left(y^{\prime}, 0\right)=\varphi_{h}(t, x)$. If $x \notin O_{k}$, then $\operatorname{dist}\left(x, \operatorname{supp} a_{k}\right) \geqslant \delta_{1}$. Hence $y^{\prime} \in B_{2}^{\prime}$, which implies that $B^{\prime}=B_{1}^{\prime} \cup B_{2}^{\prime}$.

Moreover, if $f$ is a continuous function on $\overline{B^{\prime}}$ and satisfies $f \in C^{l+\alpha}\left(\overline{B_{1}^{\prime}}\right) \cap C^{l+\alpha}\left(\overline{B_{2}^{\prime}}\right)$ for some $l \in \mathbb{N} \cup\{0\}$, then $f \in C^{l+\alpha}\left(\overline{B^{\prime}}\right)$. To see this, we may assume that $B_{1}^{\prime} \neq \emptyset$. Clearly, $f$ is $l$-times differentiable in $B^{\prime}=B_{1}^{\prime} \cup B_{2}^{\prime}$ and

$$
\sup _{y^{\prime} \in B^{\prime}}\left|\partial_{y^{\prime}}^{\theta^{\prime}} f\left(y^{\prime}\right)\right| \leqslant\|f\|_{C^{l}\left(\overline{B_{1}^{\prime}}\right)}+\|f\|_{C^{l}\left(\overline{B_{2}^{\prime}}\right)} \quad \text { for } 0 \leqslant\left|\theta^{\prime}\right| \leqslant l .
$$

Next we consider the $C^{\alpha}$ norm of $\partial_{y^{\prime}}^{l} f$. It suffices to consider the case $y_{1}^{\prime} \in B_{1}^{\prime} \backslash B_{2}^{\prime}, y_{2}^{\prime} \in B_{2}^{\prime} \backslash B_{1}^{\prime}$ if they exist. In this case, there exists a positive constant $C$, depending only on $n, r$ and $R$, such that

$$
\left|y_{1}^{\prime}-y_{2}^{\prime}\right| \geqslant C \delta_{1} \text {. }
$$

Indeed, $y_{1}^{\prime} \in B_{1}^{\prime} \backslash B_{2}^{\prime}$ implies that there exists a point $x_{1} \in O_{k} \cap U_{h}(t)$ such that $y_{1}:=$ $\left(y_{1}^{\prime}, 0\right)=\varphi_{h}\left(t, x_{1}\right)$ and $\operatorname{dist}\left(x_{1}, \operatorname{supp} a_{k}\right) \leqslant \delta_{1} / 2$. On the other hand, $y_{2}^{\prime} \in B_{2}^{\prime} \backslash B_{1}^{\prime}$ implies that there exists a point $x_{2} \in U_{h}(t) \backslash O_{k}$ such that $y_{2}:=\left(y_{2}^{\prime}, 0\right)=\varphi_{h}\left(t, x_{2}\right)$. Since $x_{2} \notin O_{k}$, we have $\operatorname{dist}\left(x_{2}, \operatorname{supp} a_{k}\right) \geqslant \delta_{1}$. Thus

$$
\left|y_{1}^{\prime}-y_{2}^{\prime}\right|=\left|y_{1}-y_{2}\right|=\left|\varphi_{h}\left(t, x_{1}\right)-\varphi_{h}\left(t, x_{2}\right)\right| \geqslant C\left|x_{1}-x_{2}\right| \geqslant C\left(\left|x_{2}-z\right|-\left|x_{1}-z\right|\right)
$$

for all $z \in \operatorname{supp} a_{k}$. In particular, we can take $z$ satisfying $\left|x_{1}-z\right|=\operatorname{dist}\left(x_{1}, \operatorname{supp} a_{k}\right)$. Hence

$$
\left|y_{1}^{\prime}-y_{2}^{\prime}\right| \geqslant C\left(\delta_{1}-\frac{\delta_{1}}{2}\right)=\frac{C}{2} \delta_{1} .
$$

This implies that $f \in C^{l+\alpha}\left(\overline{B^{\prime}}\right)$ and

$$
\|f\|_{C^{l+\alpha}\left(\overline{B^{\prime}}\right)} \leqslant C\left(\|f\|_{C^{l+\alpha}\left(\overline{B_{1}^{\prime}}\right)}+\|f\|_{C^{l+\alpha}\left(\overline{B_{2}^{\prime}}\right)}\right),
$$

where $C$ depends only on $n, r$, and $R$.

Now we return to the estimates for $\mathcal{J}, \mathcal{J}_{1}, \mathcal{J}_{2}$.

(i) Estimates on $B_{2}^{\prime}$. We first estimate $\mathcal{I}_{i, j, k, h}\left(t, s, \zeta^{\prime}\right)$ on $B_{2}^{\prime}$. By the definition of $B_{2}^{\prime}$, we see that $\left|\psi_{h}\left(t, \zeta^{\prime}, 0\right)-\psi_{k}\left(s, \xi^{\prime}, 0\right)\right|>\delta_{1} / 2$ for all $\zeta^{\prime} \in B_{2}^{\prime}$, if $\psi_{k}\left(s, \xi^{\prime}, 0\right) \in \operatorname{supp} a_{k}$. So for any multiindex $\theta$ with $|\theta|=0,1,2$, from the estimate 4.26 , we have

$$
\begin{aligned}
\left|\partial_{\zeta^{\prime}}^{\theta} \mathcal{I}_{i, j, k, h}\left(t, s, \zeta^{\prime}\right)\right| & \\
\leqslant & \int_{B^{\prime}} \mid \partial_{\zeta^{\prime}}^{\theta} K_{i, j}\left(t-s, \psi_{h}\left(t, \zeta^{\prime}, 0\right)-\psi_{k}\left(s, \xi^{\prime}, 0\right)|| \Upsilon_{1}\left(s, \xi^{\prime}\right) \mid \mathrm{d} \xi^{\prime}\right. \\
\leqslant & \sum_{0 \leqslant\left|\theta^{\prime}\right| \leqslant|\theta|} C\left(1+\left\|\psi_{h}(t, \cdot)\right\|_{C^{2+\alpha}(\bar{B})}\right)(t-s)^{-\left(n+\left|\theta^{\prime}\right|\right) / 2} \\
& \cdot \int_{B^{\prime}}\left(1+\frac{\left|\psi_{h}\left(t, \zeta^{\prime}, 0\right)-\psi_{k}\left(s, \xi^{\prime}, 0\right)\right|}{(t-s)^{1 / 2}}\right)^{-n-\left|\theta^{\prime}\right|} \mathrm{d} \xi^{\prime}\|h\|_{C} \\
\leqslant & C\left(1+\|v\|_{\left.C^{1,2+\alpha}\right)} \sum_{0 \leqslant\left|\theta^{\prime}\right| \leqslant|\theta|}(t-s)^{-\left(n+\left|\theta^{\prime}\right|\right) / 2}\left(1+\frac{\delta_{1}}{2(t-s)^{1 / 2}}\right)^{-n-\left|\theta^{\prime}\right|} \mathrm{d} \xi^{\prime}\|h\|_{C}\right. \\
\leqslant & C\|h\|_{C}
\end{aligned}
$$


where $C$ depends only on $n, r$, and $R$. Similarly, we easily have

$$
\left[\partial_{\zeta^{\prime}}^{\theta} \mathcal{I}_{i, j, k, h}(t, s, \cdot)\right]_{C^{\alpha}\left(\overline{B_{2}^{\prime}}\right)} \leqslant C\|h\|_{C}
$$

In particular,

$$
\begin{gathered}
\|\mathcal{J}(t, \cdot)\|_{C^{\alpha}\left(\overline{B_{2}^{\prime}}\right)} \leqslant C t^{1 / 2}\|h\|_{C}, \\
\left\|\mathcal{J}_{1}(t, \cdot)\right\|_{C^{\alpha}\left(\overline{B_{2}^{\prime}}\right)} \leqslant C(\eta t)^{1 / 2}\|h\|_{C}, \\
\left\|\mathcal{J}_{2}(t, \cdot)\right\|_{C^{2+\alpha}\left(\overline{B_{2}^{\prime}}\right)} \leqslant C(\eta t)^{-1 / 2}\|h\|_{C} .
\end{gathered}
$$

(ii) Estimates on $B_{1}^{\prime}$. In order to obtain the desired estimates, it suffices to show that

$$
\begin{gathered}
\left\|\mathcal{I}_{i, j, k, h}(t, s, \cdot)\right\|_{C^{\alpha}\left(\overline{B_{1}^{\prime}}\right)} \leqslant C(t-s)^{-1 / 2}\|h\|_{C^{0, \alpha}}, \\
\left\|\mathcal{I}_{i, j, k, h}(t, s, \cdot)\right\|_{C^{2+\alpha}\left(\overline{B_{1}^{\prime}}\right)} \leqslant C(t-s)^{-3 / 2}\|h\|_{C^{0, \alpha}} .
\end{gathered}
$$

If $\zeta^{\prime} \in B_{1}$, then there exists a unique $w^{\prime} \in B^{\prime}$ such that $\psi_{h}\left(t, \zeta^{\prime}, 0\right)=\psi_{k}\left(t, w^{\prime}, 0\right)$, or $w=$ $\left(w^{\prime}, 0\right)=\varphi_{k}\left(t, \psi_{h}\left(t, \zeta^{\prime}, 0\right)\right)$. Note that $w^{\prime}$ is a function of $t$ and $\zeta^{\prime}$, but for simplicity of notation, we just write $w^{\prime}$, or $w^{\prime}\left(\zeta^{\prime}\right)$. From the estimate (4.22), we have

$$
\left\|w^{\prime}\right\|_{C^{1,2+\alpha}\left([0, T] \times \overline{B^{\prime}}\right)} \leqslant C\left(1+\|v\|_{C^{1,2+\alpha}}^{2}\right) .
$$

As in (i), for any multi-index $\theta$ with $|\theta|=0,1,2$, from (4.26, we have

$$
\begin{aligned}
& \left|\partial_{\zeta^{\prime}}^{\theta} \mathcal{I}_{i, j, k, h}\left(t, s, \zeta^{\prime}\right)\right| \\
& \quad \leqslant \int_{\mathbb{R}^{n-1}} \mid \partial_{\zeta^{\prime}}^{\theta} K_{i, j}\left(t-s, \psi_{h}\left(t, \zeta^{\prime}, 0\right)-\psi_{k}\left(s, \xi^{\prime}, 0\right)|| \Upsilon_{1}\left(s, \xi^{\prime}\right) \mid \mathrm{d} \xi^{\prime}\right. \\
& \quad \leqslant C\left(1+\left\|\psi_{h}(t, \cdot)\right\|_{C^{2+\alpha}\left(\overline{B^{\prime}}\right)}\right) \sum_{\left|\theta^{\prime}\right|=0}^{|\theta|} \int_{\mathbb{R}^{n-1}} \mid\left(\partial_{x}^{\theta^{\prime}} K_{i, j}\right)\left(t-s, \psi_{k}\left(t, w^{\prime}, 0\right)-\psi_{k}\left(s, \xi^{\prime}, 0\right) \mid \mathrm{d} \xi^{\prime}\|h\|_{C}\right. \\
& \quad \leqslant C\left(1+\|v\|_{\left.C^{1,2+\alpha}\right)} \sum_{\left|\theta^{\prime}\right|=0}^{|\theta|}(t-s)^{-\left(n+\left|\theta^{\prime}\right|\right) / 2} \int_{\mathbb{R}^{n-1}}\left(1+C \frac{\left|w^{\prime}-\xi^{\prime}\right|}{(t-s)^{1 / 2}}\right)^{-n-\left|\theta^{\prime}\right|} \mathrm{d} \xi^{\prime}\|h\|_{C}\right. \\
& \quad \leqslant C\left(1+\|v\|_{C^{1,2+\alpha}}\right)(t-s)^{-(1+|\theta|) / 2}\|h\|_{C} .
\end{aligned}
$$

So we obtain

$$
\begin{gathered}
\left\|\mathcal{I}_{i, j, k, h}(t, s, \cdot)\right\|_{C\left(\overline{B_{1}^{\prime}}\right)} \leqslant C(t-s)^{-1 / 2}\|h\|_{C}, \\
\left\|\mathcal{I}_{i, j, k, h}(t, s, \cdot)\right\|_{C^{2}\left(\overline{B_{1}^{\prime}}\right)} \leqslant C(t-s)^{-3 / 2}\|h\|_{C} .
\end{gathered}
$$

Thus it suffices to estimate $\left[\mathcal{I}_{i, j, k, h}(t, s, \cdot)\right]_{C^{\alpha}\left(\overline{B_{1}^{\prime}}\right)}$ and $\left[\partial_{\zeta^{\prime}}^{2} \mathcal{I}_{i, j, k, h}(t, s, \cdot)\right]_{C^{\alpha}\left(\overline{B_{1}^{\prime}}\right)}$; we only handle the first, as the other is similar.

We make use of the $\alpha$-Hölder continuity of $\Upsilon_{1}$. For this purpose, by changing variable as $w^{\prime}-\xi^{\prime}=z^{\prime}$, we rewrite $\mathcal{I}_{i, j, k, h}\left(t, s, \zeta^{\prime}\right)$ as

$$
\mathcal{I}_{i, j, k, h}\left(t, s, \zeta^{\prime}\right)=\int_{\mathbb{R}^{n-1}} K_{i, j}\left(t-s, \psi_{k}\left(t, w^{\prime}, 0\right)-\psi_{k}\left(s, w^{\prime}-z^{\prime}, 0\right)\right) \Upsilon_{1}\left(s, w^{\prime}-z^{\prime}\right) \mathrm{d} z^{\prime} .
$$


For $\zeta_{1}^{\prime}, \zeta_{2}^{\prime} \in B_{1}^{\prime}$, we set $w_{1}^{\prime}=w_{1}\left(\zeta^{\prime}\right), w_{2}^{\prime}=w_{2}^{\prime}\left(\zeta_{2}^{\prime}\right)$. We also set

$$
\Upsilon_{2}\left(t, s, w^{\prime}, z^{\prime}\right):=\psi_{k}\left(t, w^{\prime}, 0\right)-\psi_{k}\left(s, w^{\prime}-z^{\prime}\right) .
$$

Then

$$
\begin{aligned}
\left|\Upsilon_{2}\left(t, s, w_{1}^{\prime}, z^{\prime}\right)-\Upsilon_{2}\left(t, s, w_{2}^{\prime}, z^{\prime}\right)\right| & =\left|\int_{0}^{1}\left\langle w_{1}^{\prime}-w_{2}^{\prime},\left(\nabla_{w^{\prime}} \Upsilon_{2}\right)\left(t, s, \tau w_{1}^{\prime}+(1-\tau) w_{2}^{\prime}, z^{\prime}\right)\right\rangle^{\prime} \mathrm{d} \tau\right| \\
& \leqslant C\left|\zeta_{1}^{\prime}-\zeta_{2}^{\prime}\right|\left((t-s)^{(1+\alpha) / 2}+\left|z^{\prime}\right|\right) .
\end{aligned}
$$

So from the estimate [4.26), we have

$$
\begin{aligned}
\left|\mathcal{I}_{i, j, k, h}\left(t, s, \zeta_{1}^{\prime}\right)-\mathcal{I}_{i, j, k, h}\left(t, s, \zeta_{2}^{\prime}\right)\right| & \\
\leqslant & \int_{\mathbb{R}^{n-1}}\left|K_{i, j}\left(t-s, \Upsilon_{2}\left(t, s, w_{1}^{\prime}, z^{\prime}\right)\right)-K_{i, j}\left(t-s, \Upsilon_{2}\left(t, s, w_{2}^{\prime}, z^{\prime}\right)\right)\right|\left|\Upsilon_{1}\left(s, w_{1}^{\prime}-z^{\prime}\right)\right| \mathrm{d} z^{\prime} \\
& \quad+\int_{\mathbb{R}^{n-1}}\left|K_{i, j}\left(t-s, \Upsilon_{2}\left(t, s, w_{2}^{\prime}, z^{\prime}\right)\right)\right|\left|\Upsilon_{1}\left(s, w_{1}^{\prime}-z^{\prime}\right)-\Upsilon_{1}\left(s, w_{2}^{\prime}-z^{\prime}\right)\right| \mathrm{d} z^{\prime} \\
\leqslant & C\|h\|_{C} \int_{\mathbb{R}^{n-1}} \int_{0}^{1} \mid\left\langle\Upsilon_{2}\left(t, s, w_{1}^{\prime}, z^{\prime}\right)-\Upsilon_{2}\left(t, s, w_{2}^{\prime}, z^{\prime}\right),\right. \\
& \left.\quad\left(\nabla_{x} K_{i, j}\right)\left(t-s, \tau \Upsilon_{2}\left(t, s, w_{1}^{\prime}, z^{\prime}\right)+(1-\tau) \Upsilon_{2}\left(t, s, w_{2}^{\prime}, z^{\prime}\right)\right)\right\rangle \mid \mathrm{d} \tau \mathrm{d} z^{\prime} \\
& +C\|h\|_{C^{0, \alpha}}\left|w_{1}^{\prime}-w_{2}^{\prime}\right|^{\alpha}(t-s)^{-n / 2} \int_{\mathbb{R}^{n-1}}\left(1+C \frac{\left|z^{\prime}\right|}{(t-s)^{1 / 2}}\right)^{-n} \mathrm{~d} z^{\prime} \\
\leqslant & C\|h\|_{C}\left|\zeta_{1}^{\prime}-\zeta_{2}^{\prime}\right|(t-s)^{-(n+1) / 2} \int_{\mathbb{R}^{n-1}}\left((t-s)^{(1+\alpha) / 2}+\left|z^{\prime}\right|\right)\left(1+C \frac{\left|z^{\prime}\right|}{(t-s)^{1 / 2}}\right)^{-n-1} \mathrm{~d} z^{\prime} \\
& +C\|h\|_{C^{0, \alpha}}\left|\zeta_{1}^{\prime}-\zeta_{2}^{\prime}\right|^{\alpha}(t-s)^{-1 / 2} \\
\leqslant & C\left|\zeta_{1}^{\prime}-\zeta_{2}^{\prime}\right|^{\alpha}(t-s)^{-1 / 2}\|h\|_{C^{0, \alpha}},
\end{aligned}
$$

which is the desired estimate. We have just established the estimates (4.37)-(4.39).

We are now in a position to show the optimal regularity $\|\mathcal{J}(t, \cdot)\|_{C^{1+\alpha}\left(\overline{B^{\prime}}\right)}$. We use the relation $C^{1+\alpha}\left(\overline{B^{\prime}}\right)=\mathcal{C}^{1+\alpha}\left(\overline{B^{\prime}}\right)$ (equivalent norms), where $\mathcal{C}^{l}\left(\overline{B^{\prime}}\right), 0<l<3$, are the Hölder-Zygmund spaces defined as follows:

$$
\mathcal{C}^{l}\left(\overline{B^{\prime}}\right):=\left\{f \in C\left(\overline{B^{\prime}}\right) ;\|f\|_{\mathcal{C}^{l}\left(\overline{B^{\prime}}\right)}:=\|f\|_{C\left(\overline{B^{\prime}}\right)}+[f]_{\mathcal{C}^{l}\left(\overline{B^{\prime}}\right)}<\infty\right\},
$$

where

$$
[f]_{\mathcal{C}^{l}\left(\overline{B^{\prime}}\right)}:=\sup _{x, y \in \overline{B^{\prime}}, x \neq y} \frac{\left|f(x)-3 f\left(\frac{2 x+y}{3}\right)+3 f\left(\frac{x+2 y}{3}\right)-f(y)\right|}{|x-y|^{l}} .
$$

The advantage of the Hölder-Zygmund spaces is that derivatives do not appear in the definition of its norms. We have the relation $C^{l}\left(\overline{B^{\prime}}\right)=\mathcal{C}^{l}\left(\overline{B^{\prime}}\right)$ with equivalent norms if $l$ is not an integer; see A. Lunardi [13] or T. Runst and W. Sickel [21]. 
It suffices to estimate $[\mathcal{J}(t, \cdot)]_{\mathcal{C}^{1+\alpha}}$. Let $\zeta_{1}^{\prime}, \zeta_{2}^{\prime} \in B^{\prime}$ with $\zeta_{1}^{\prime} \neq \zeta_{2}^{\prime}$. We first consider the case where $\left|\zeta_{1}^{\prime}-\zeta_{2}^{\prime}\right| \leqslant \frac{1}{2} t^{1 / 2}$. Since $\mathcal{J}=\mathcal{J}_{1}+\mathcal{J}_{2}$, from 4.38 and 4.39 we have

$$
\begin{aligned}
\mid \mathcal{J}\left(t, \zeta_{1}^{\prime}\right)-3 \mathcal{J}\left(t, \frac{2 \zeta_{1}^{\prime}+\zeta_{2}^{\prime}}{3}\right) & +3 \mathcal{J}\left(t, \frac{\zeta_{1}^{\prime}+2 \zeta_{2}^{\prime}}{3}\right)-\mathcal{J}\left(t, \zeta_{2}^{\prime}\right) \mid \\
\leqslant & {\left[\mathcal{J}_{1}(t, \cdot)\right]_{\mathcal{C}^{\alpha}}\left|\zeta_{1}^{\prime}-\zeta_{2}^{\prime}\right|^{\alpha}+\left[\mathcal{J}_{2}(t, \cdot)\right]_{\mathcal{C}^{2+\alpha}}\left|\zeta_{1}^{\prime}-\zeta_{2}^{\prime}\right|^{2+\alpha} } \\
\leqslant & \leqslant\left[\mathcal{J}_{1}(t, \cdot)\right]_{C^{\alpha}}\left|\zeta_{1}^{\prime}-\zeta_{2}^{\prime}\right|^{\alpha}+C\left[\mathcal{J}_{2}(t, \cdot)\right]_{C^{2+\alpha}}\left|\zeta_{1}^{\prime}-\zeta_{2}^{\prime}\right|^{2+\alpha} \\
& \leqslant C\|h\|_{C^{0, \alpha}(\eta t)^{1 / 2}\left|\zeta_{1}^{\prime}-\zeta_{2}^{\prime}\right|^{\alpha}+C\|h\|_{C^{0, \alpha}}(\eta t)^{-1 / 2}\left|\zeta_{1}^{\prime}-\zeta_{2}^{\prime}\right|^{2+\alpha}}
\end{aligned}
$$

for $\eta \in(0,1)$. So if we set $\eta=\left|\zeta_{1}^{\prime}-\zeta_{2}^{\prime}\right|^{2} / t$, then

$$
\left|\mathcal{J}\left(t, \zeta_{1}^{\prime}\right)-3 \mathcal{J}\left(t, \frac{2 \zeta_{1}^{\prime}+\zeta_{2}^{\prime}}{3}\right)+3 \mathcal{J}\left(t, \frac{\zeta_{1}^{\prime}+2 \zeta_{2}^{\prime}}{3}\right)-\mathcal{J}\left(t, \zeta_{2}^{\prime}\right)\right| \leqslant C\|h\|_{C^{0, \alpha}}\left|\zeta_{1}^{\prime}-\zeta_{2}^{\prime}\right|^{1+\alpha}
$$

for any $\zeta_{1}^{\prime}, \zeta_{2}^{\prime} \in B^{\prime}$ with $\left|\zeta_{1}^{\prime}-\zeta_{2}^{\prime}\right| \leqslant \frac{1}{2} t^{1 / 2}$. If $\left|\zeta_{1}^{\prime}-\zeta_{2}^{\prime}\right| \geqslant \frac{1}{2} t^{1 / 2}$, then from 4.37 ,

$$
\begin{aligned}
\left|\mathcal{J}\left(t, \zeta_{1}^{\prime}\right)-3 \mathcal{J}\left(t, \frac{2 \zeta_{1}^{\prime}+\zeta_{2}^{\prime}}{3}\right)+3 \mathcal{J}\left(t, \frac{\zeta_{1}^{\prime}+2 \zeta_{2}^{\prime}}{3}\right)-\mathcal{J}\left(t, \zeta_{2}^{\prime}\right)\right| \\
\leqslant[\mathcal{J}(t, \cdot)]_{\mathcal{C}^{\alpha}}\left|\zeta_{1}^{\prime}-\zeta_{2}^{\prime}\right|^{\alpha} \leqslant C\|h\|_{C^{0, \alpha}} t^{1 / 2}\left|\zeta_{1}^{\prime}-\zeta_{2}^{\prime}\right|^{\alpha} \leqslant C\|h\|_{C^{0, \alpha}}\left|\zeta_{1}^{\prime}-\zeta_{2}^{\prime}\right|^{1+\alpha} .
\end{aligned}
$$

Hence $\mathcal{J} \in \mathcal{C}^{1+\alpha}\left(\overline{B^{\prime}}\right)$, equivalently, $\mathcal{J} \in C^{1+\alpha}\left(\overline{B^{\prime}}\right)$. This completes the proof.

\subsection{Remark on the mild solution of the Navier-Stokes equation}

In this section, we shall construct a mild solution of the Navier-Stokes equation with initial velocity $u_{0} \in C^{\alpha}\left(\mathbb{R}^{n}\right)$ and with layer potential term $h \mathcal{H}_{\left\llcorner\Gamma_{t}\right.}^{n-1}$. Thanks to the estimates for the layer potential term established in the previous section, we can obtain appropriate regularity for solutions in the tangential directions to $\Gamma_{t}$. We recall that the mild solution of the Navier-Stokes equation which we consider here is a solution of the integral equation

$$
u(t)=e^{t \Delta} u_{0}-\int_{0}^{t} e^{(t-s) \Delta} \mathbf{P} \nabla \cdot u \otimes u \mathrm{~d} s+\int_{0}^{t} e^{(t-s) \Delta} \mathbf{P} h \mathcal{H}_{\left\llcorner\Gamma_{s}\right.}^{n-1} \mathrm{~d} s .
$$

Now let $\alpha \in(0,1)$. Assume that $u_{0} \in C^{\alpha}\left(\mathbb{R}^{n}\right)$ satisfies $\nabla \cdot u_{0}=0$ and $d_{0}$ is the distance function of a $C^{2+\alpha}$ hypersurface $\Gamma_{0}$. Let $R \geqslant 1$ be a given number and $T_{0}(<1)$ be the number given by Proposition 6.1 in the Appendix, depending only on $n, d_{0}$, and $R$. Let $T_{1} \in\left(0, T_{0}\right]$ and $\left\{\Gamma_{t}\right\}_{0 \leqslant t \leqslant T_{1}}$ be an evolving hypersurface belonging to $\mathcal{S}\left(\alpha, R, T_{1}, d_{0}\right)$. Then we have the following proposition.

Proposition 4.2 There exists a $T \leqslant T_{1}$ such that there exists a unique mild solution $u \in$ $C^{\alpha / 2, \alpha}\left([0, T] \times \mathbb{R}^{n}\right)$. The existence time $T$ can be taken uniformly in $\mathcal{S}\left(\alpha, R, T_{1}, d_{0}\right)$. Moreover, this solution satisfies the following estimates:

$$
\begin{gathered}
\|u\|_{C^{\alpha / 2, \alpha}\left(\left[0, T \times \mathbb{R}^{n}\right)\right.} \leqslant C\left\|u_{0}\right\|_{C^{\alpha}\left(\mathbb{R}^{n}\right)}+C_{1} T^{(1-\alpha) / 2}\|h\|_{C([0, T] \times \bar{D})}, \\
\sup _{0<t<T} t^{(1-\alpha) / 2}\|u(t, \cdot)\|_{C^{1}\left(\Gamma_{t}\right)}+\sup _{0<t<T} t^{1 / 2}\|u(t, \cdot)\|_{C^{1+\alpha}\left(\Gamma_{t}\right)^{r}} \\
\leqslant C\left\|u_{0}\right\|_{C^{\alpha}\left(\mathbb{R}^{n}\right)}+C_{2} T^{(1-\alpha) / 2}\|h\|_{C^{0, \alpha}([0, T] \times \bar{D})},
\end{gathered}
$$

where $C=C(n, \alpha), C_{1}\left(n, \alpha, d_{0}, R\right)$, and $C_{2}\left(n, \alpha, d_{0}, R\right)$. 
Proof. We will follow the contraction argument by T. Kato [10]. Since this argument is well-known, we only outline it. Set

$$
\begin{aligned}
& F_{0}:=e^{t \Delta} u_{0}, \\
& B(f, g):=\int_{0}^{t} e^{(t-s) \Delta} \mathbf{P} \nabla \cdot f \otimes g \mathrm{~d} s, \quad f, g \in C^{0, \alpha}\left([0, T] \times \mathbb{R}^{n}\right), \\
& F:=\int_{0}^{t} e^{(t-s) \Delta} \mathbf{P} h \mathcal{H}_{\left\llcorner\Gamma_{s}\right.}^{n-1} \mathrm{~d} s .
\end{aligned}
$$

From the pointwise estimate of the kernel $e^{t \Delta} \mathbf{P} \nabla$. in Lemma 4.1 it is not difficult to deduce the estimate

$$
\|B(f, g)\|_{C^{\alpha / 2, \alpha}\left([0, T] \times \mathbb{R}^{n}\right)} \leqslant C T^{1 / 2}\|f\|_{C^{0, \alpha}\left([0, T] \times \mathbb{R}^{n}\right)}\|g\|_{C^{0, \alpha}\left([0, T] \times \mathbb{R}^{n}\right)}
$$

where $C$ depends only on $n$ and $\alpha$. Combining the estimates in Lemma 4.2 and Proposition 4.1, we easily see that

$$
G(w):=F_{0}-B(w, w)+F
$$

is a contraction mapping from the closed ball in $C^{\alpha / 2, \alpha}\left([0, T] \times \mathbb{R}^{n}\right)$ with radius $2\left(\left\|F_{0}\right\|_{C^{0, \alpha}\left([0, T] \times \mathbb{R}^{n}\right)}+\|F\|_{C^{0, \alpha}\left([0, T] \times \mathbb{R}^{n}\right)}\right)$ into itself for sufficiently small $T \leqslant T_{1}$. This implies the time-local existence of the unique solution $u$. Note that the existence time can be taken uniformly in $\mathcal{S}\left(\alpha, R, T_{1}, d_{0}\right)$ since the constants in Proposition 4.1 do not depend on the family of hypersurfaces in $\mathcal{S}\left(\alpha, R, T_{1}, d_{0}\right)$. The estimate 4.51 ) is obvious. We shall show the estimate 4.52 ). Note that

$$
\left\|\partial_{x} F_{0}(t, \cdot)\right\|_{C\left(\mathbb{R}^{n}\right)} \leqslant C t^{-(1-\alpha) / 2}\left\|u_{0}\right\|_{C^{\alpha}}, \quad\left[\partial_{x} F_{0}(t, \cdot)\right]_{C^{\alpha}\left(\mathbb{R}^{n}\right)} \leqslant C t^{-1 / 2}\left\|u_{0}\right\|_{C^{\alpha}} .
$$

As for the nonlinear term $B(f, g)$, we recall the estimates

$$
\left\|\nabla e^{t \Delta} \mathbf{P} \nabla \cdot f\right\|_{C\left(\mathbb{R}^{n}\right)} \leqslant C t^{-1}\|f\|_{C\left(\mathbb{R}^{n}\right)}, \quad\left\|\nabla e^{t \Delta} \mathbf{P} \nabla \cdot f\right\|_{C\left(\mathbb{R}^{n}\right)} \leqslant C t^{-1 / 2}\|\nabla f\|_{C\left(\mathbb{R}^{n}\right)},
$$

for any $f \in\left(C^{1}\left(\mathbb{R}^{n}\right)\right)^{n \times n}$; for example, see [14, Corollary 3.1]. By interpolating, we have

$$
\left\|\nabla e^{t \Delta} \mathbf{P} \nabla \cdot f\right\|_{C\left(\mathbb{R}^{n}\right)} \leqslant C t^{-1+\alpha / 2}\|f\|_{C^{\alpha}} .
$$

Hence

$$
\begin{aligned}
\|\nabla B(f, g)(t, \cdot)\|_{C\left(\mathbb{R}^{n}\right)} & \leqslant C \int_{0}^{t}(t-s)^{-1+\alpha / 2}\|f \otimes g(s)\|_{C^{\alpha}\left(\mathbb{R}^{n}\right)} \mathrm{d} s \\
& \leqslant C t^{\alpha / 2}\|f\|_{C^{0, \alpha}\left([0, T] \times \mathbb{R}^{n}\right)}\|g\|_{C^{0, \alpha}\left([0, T] \times \mathbb{R}^{n}\right)},
\end{aligned}
$$

thus

$$
\|\nabla B(f, g)\|_{C\left([0, T] \times \mathbb{R}^{n}\right)} \leqslant C T^{\alpha / 2}\|f\|_{C^{0, \alpha}\left([0, T] \times \mathbb{R}^{n}\right)}\|g\|_{C^{0, \alpha}\left([0, T] \times \mathbb{R}^{n}\right)} .
$$

Next since the Helmholtz projection $\mathbf{P}$ is bounded in the homogeneous counterpart of $C^{\alpha}\left(\mathbb{R}^{n}\right)$, we see that

$$
[\nabla B(f, g)(t, \cdot)]_{C^{\alpha}\left(\mathbb{R}^{n}\right)} \leqslant C\left[\nabla \int_{0}^{t} e^{(t-s) \Delta} \nabla \cdot f \otimes g \mathrm{~d} s\right]_{C^{\alpha}\left(\mathbb{R}^{n}\right)} .
$$

Then, from the maximal regularity estimates for the heat equation (see [13]), we easily obtain

$$
\|\nabla B(f, g)\|_{C^{0, \alpha}\left([0, T] \times \mathbb{R}^{n}\right)} \leqslant C\|f\|_{C^{0, \alpha}\left([0, T] \times \mathbb{R}^{n}\right)}\|g\|_{C^{0, \alpha}\left([0, T] \times \mathbb{R}^{n}\right)} .
$$

Combining the above estimates with the estimate for $F$ in the tangential directions to $\Gamma_{t}$ established in Proposition 4.1, we obtain the desired estimates. This completes the proof. 


\section{Construction of the solution for the free boundary problem}

Now we return to the problem $(\overline{\mathrm{FBP}})$. In this section, we shall prove the main theorem. Let $u_{0} \in$ $C^{\alpha}\left(\mathbb{R}^{n}\right)$ satisfy $\nabla \cdot u_{0}=0$. Let $\Gamma_{0}$ be a $C^{2+\alpha}$ hypersurface which is the boundary of a bounded domain $\Omega_{0}$ and let $d_{0}$ be the signed distance function of $\Gamma_{0}$. We set $F_{0}(t, \cdot)=e^{t \Delta_{0}} u_{0}$ and

$$
M:=2\left(\left\|F_{0}\right\|_{C^{0, \alpha}\left([0, \infty) \times \mathbb{R}^{n}\right)}+\sup _{t>0} t^{(1-\alpha) / 2}\left\|\partial_{x} F_{0}(t, \cdot)\right\|_{C\left(\mathbb{R}^{n}\right)}+\sup _{t>0} t^{1 / 2}\left[\partial_{x} F_{0}(t, \cdot)\right]_{C^{\alpha}\left(\mathbb{R}^{n}\right)}\right)
$$

Recall that $\mathcal{U}_{M}$ is the closed subset of $C^{0, \alpha}\left([0, T] \times \mathbb{R}^{n}\right)$ defined as

$$
\begin{aligned}
\mathcal{U}_{M}=\left\{u(t, x) \in C^{0, \alpha}\left([0, T] \times \mathbb{R}^{n}\right) ; u(t, \cdot) \in C^{1+\alpha}\left(\mathbb{R}^{n}\right), L_{u}:=\sup _{0<t<T}\|u(t, \cdot)\|_{C^{\alpha}\left(\mathbb{R}^{n}\right)}\right. \\
\left.+\sup _{0<t<T} t^{(1-\alpha) / 2}\left\|\partial_{x} u(t, \cdot)\right\|_{C\left(\mathbb{R}^{n}\right)}+\sup _{0<t<T} t^{1 / 2}\left[\partial_{x} u(t, \cdot)\right]_{C^{\alpha}\left(\mathbb{R}^{n}\right)} \leqslant M\right\} .
\end{aligned}
$$

From Proposition 3.1, there exists a positive $T_{1}$ such that for any $u \in \mathcal{U}_{M}$, there exists a unique family $\left\{\Gamma_{t}^{u}\right\}_{0 \leqslant t \leqslant T_{1}}$ of hypersurfaces evolving via the convected mean curvature equation (3.1) starting from $\Gamma_{0}$. Moreover, this $\left\{\Gamma_{t}^{u}\right\}_{0 \leqslant t \leqslant T_{1}}$ belongs to $\mathcal{S}\left(\alpha, R, T_{1}, d_{0}\right)$ with $R=\left\|d_{0}\right\|_{C^{2+\alpha}}+$ $2 C\left(\left\|d_{0}\right\|_{C^{2+\alpha}(\bar{D})}, K_{f}, M\right)$; see 3.16 . Let $v$ be the signed distance function of $\left\{\Gamma_{t}^{u}\right\}_{0 \leqslant t \leqslant T_{1}}$. From Proposition 3.2. we have

$$
\sup _{0<t<T_{1}} t^{1 / 2}\left\|\partial_{x}^{3} v(t, \cdot)\right\|_{C^{\alpha}\left(\overline{D^{\prime}}\right)}<\infty
$$

for any open set $D^{\prime} \subset \subset D$. Let $T_{2}:=\min \left(T_{1}, T_{0}\right)$, where $T_{0}$ is the number given by Proposition 6.1. We set

$$
C_{4}:=\sup _{0<t<T_{2}} t^{1 / 2}\left\|\partial_{x}^{3} v(t, \cdot)\right\|_{C^{\alpha}\left(\bigcup_{k=1}^{m} \bar{O}_{k}\right)},
$$

where $\left\{O_{k}\right\}_{k=1}^{m}$ is the family of open sets given by Proposition 6.1. Note that $C_{4}$ is bounded uniformly with respect to functions belonging to $\mathcal{U}_{M}$. Set

$$
F^{u}(t, \cdot):=\int_{0}^{t} e^{(t-s) \Delta} \mathbf{P} \sigma_{1} H^{u} v^{u} \mathcal{H}_{\left\llcorner\Gamma_{s}^{u}\right.} \mathrm{d} s,
$$

where $H^{u}, v^{u}$ are the mean curvature and the exterior unit normal vector of $\Gamma_{t}^{u}$, respectively. We remark that for the signed distance function $v$, the exterior unit normal vector $v^{u}(t, x)$ and the mean curvature $H^{u}(t, x)$ of the surface $\Gamma_{t}$ are given by

$$
\begin{aligned}
v^{u}(t, x) & =\nabla_{x} v(t, x), \\
H^{u}(t, x) & =-\frac{1}{n-1} \operatorname{div} v(t, x)=-\frac{1}{n-1} \Delta v(t, x) .
\end{aligned}
$$

Since $v$ is a function on $[0, T] \times \bar{D}, v$ and $H^{u}$ can also be regarded as functions on $[0, T] \times \bar{D}$. In particular, if $\left\{\Gamma_{t}\right\}_{0 \leqslant t \leqslant T}$ is an evolving hypersurface belonging to $\mathcal{S}\left(\alpha, R, T, d_{0}\right)$, then the mean curvature vector $H^{u} v^{u}$ belongs to $C^{0, \alpha}([0, T] \times \bar{D})$ as a function on $[0, T] \times \bar{D}$. Moreover, if the above $v$ satisfies $\sup _{0<t<T} t^{1 / 2}\left\|\partial_{x} v^{3}(t, \cdot)\right\|_{C^{\alpha}\left(\overline{D^{\prime}}\right)}<\infty$ for an open set $D^{\prime} \subset \subset D$, then

$$
\sup _{0<t<T} t^{1 / 2}\left\|\partial_{x} H^{u}(t, \cdot)\right\|_{C^{\alpha}\left(\overline{D^{\prime}}\right)} \leqslant C \sup _{0<t<T} t^{1 / 2}\left\|\partial_{x}^{3} v(t, \cdot)\right\|_{C^{\alpha}\left(\overline{D^{\prime}}\right)} .
$$


From Proposition 4.1, the function $F^{u}$ satisfies

$$
\begin{aligned}
& \left\|F^{u}\right\|_{C^{\alpha / 2, \alpha}\left(\left[0, T_{2}\right] \times \bar{D}\right)} \leqslant \sigma_{1} C T^{(1-\alpha) / 2}, \\
& \sup _{0 \leqslant t \leqslant T_{2}}\left\|F^{u}(t)\right\|_{C^{1+\alpha}\left(\Gamma_{t}\right)} \leqslant \sigma_{1} C,
\end{aligned}
$$

where $C$ depends only on $n, \alpha, R$, and $\Gamma_{0}$. Since $F^{u}(t, \cdot)$ belongs to $C^{1+\alpha}\left(\Gamma_{t}^{u}\right)$ for each $t \in\left(0, T_{2}\right]$, we can construct a function in $C^{1+\alpha}\left(\mathbb{R}^{n}\right)$ as an extension of $\gamma_{\Gamma_{t}^{u}} F^{u}(t, \cdot)$, where $\gamma_{\Gamma_{t}^{u}}$ is the restriction operator to $\Gamma_{t}^{u}$. We fix the extension as follows. Let $\left\{\varphi_{k}\right\}_{k=1}^{m},\left\{\psi_{k}\right\}_{k=1}^{m}$ be local coordinate transforms of $\left\{\Gamma_{t}^{u}\right\}_{0 \leqslant t \leqslant T_{2}}$ and $\left\{\hat{O}_{k}\right\}_{k=1}^{m}$ be the family of open sets given by Proposition 6.1. Let $\left\{a_{k}\right\}_{k=1}^{m}$ be a partition of unity for $\bigcup_{k=1}^{m} \hat{O}_{k}$ subordinate to $\left\{O_{k}\right\}_{k=1}^{m}$. Set

$$
\begin{aligned}
& E_{1, k}^{v}\left(F^{u}\right)(t, y):=F^{u}\left(t, \psi_{k}\left(t, y^{\prime}, 0\right)\right), \quad y=\left(y^{\prime}, y^{(n)}\right) \in \mathbb{R}^{n},|y| \leqslant 1, \\
& E_{2, k}^{v}\left(F^{u}\right)(t, x):= \begin{cases}a_{k}(x) E_{1, k}^{v}\left(F^{u}\right)\left(t, \varphi_{k}(t, x)\right), & x \in O_{k}, \\
0, & x \notin O_{k} .\end{cases}
\end{aligned}
$$

Moreover, we set

$$
E^{v}\left(F^{u}\right)(t, x):=\sum_{k=1}^{m} E_{2, k}^{v}\left(F^{u}\right)(t, x) .
$$

Then obviously $E^{v}\left(F^{u}\right)(t, x)=F^{u}(t, x)$ for all $x \in \Gamma_{t}$ and

$$
\begin{aligned}
& \left\|E^{v}\left(F^{u}\right)\right\|_{C^{\alpha / 2, \alpha}\left(\left[0, T_{2}\right] \times \bar{D}\right)} \leqslant C_{5} \sigma_{1} C T^{(1-\alpha) / 2}, \\
& \sup _{0 \leqslant t \leqslant T_{2}}\left\|E^{v}\left(F^{u}\right)(t)\right\|_{C^{1+\alpha}\left(\mathbb{R}^{n}\right)} \leqslant C_{5} \sigma_{1} C,
\end{aligned}
$$

where $C_{5}$ depends only on $n, R$, and $\Gamma_{0}$. Note that the partition of unity $\left\{a_{k}\right\}_{k=1}^{m}$ can be taken uniformly in $\bigcup_{0<T \leqslant T_{2}} \mathcal{S}\left(\alpha, R, T, d_{0}\right)$. Moreover, although the extension $E^{v}$ depends on $\left\{\Gamma_{t}^{u}\right\}_{0 \leqslant t \leqslant T_{2}}$, the constant $C_{5}$ is independent of the evolving hypersurface belonging to $\bigcup_{0<T \leqslant T_{2}} \mathcal{S}\left(\alpha, R, T, d_{0}\right)$. Let $\Psi_{0}(u)$ be the unique mild solution with initial velocity $u_{0}$ and with layer potential $\sigma_{1} H^{u} v^{u} \mathcal{H}_{\left\llcorner\Gamma_{t}\right.}^{n-1}$, i.e.,

$$
\Psi_{0}(u)=F_{0}+F^{u} .
$$

Finally, we set

$$
\Psi(u)(t, x):=F_{0}+E^{v}\left(F^{u}\right) .
$$

Clearly, $\Psi$ is a mapping on $\mathcal{U}_{M}$. We shall show that $\Psi$ is a contraction mapping $\mathcal{U}_{M}$ into itself. From the estimates 5.11, (5.12) and the definition of $M$, we have

$$
L_{\Psi(u)} \leqslant M / 2+C_{5} \sigma_{1} C T^{(1-\alpha) / 2} .
$$

Since each constant above is independent of $T$, we see that for sufficiently small $T \leqslant T_{2}, \Psi$ maps $\mathcal{U}_{M}$ into itself. Next we shall show that $\Psi$ is a contraction mapping. Let $u, \tilde{u} \in \mathcal{U}_{M}$, and $v, \tilde{v}$ be the signed distance functions of $\left\{\Gamma_{t}^{u}\right\}_{0 \leqslant t \leqslant T},\left\{\Gamma_{t}^{\tilde{u}}\right\}_{0 \leqslant t \leqslant T}$, respectively. From the construction of the map $\Psi$, we see that

$$
\begin{aligned}
\Psi(u)(t, x)-\Psi(\tilde{u})(t, x) & =E^{v}\left(F^{u}\right)(t, x)-E^{\tilde{v}}\left(F^{\tilde{u}}\right)(t, x) \\
& =\sum_{k=1}^{m} a_{k}(x)\left\{F^{u}\left(t, \psi_{k}\left(t, \varphi_{k}^{\prime}(t, x), 0\right)\right)-F^{\tilde{u}}\left(t, \tilde{\psi}_{k}\left(t, \tilde{\varphi}_{k}^{\prime}(t, x), 0\right)\right)\right\} .
\end{aligned}
$$


Thus it suffices to estimate

$$
\left\|F^{u}\left(\cdot, \psi_{k}\left(\cdot, \varphi_{k}^{\prime}(\cdot, \cdot), 0\right)\right)-F^{\tilde{u}}\left(\cdot, \tilde{\psi}_{k}\left(\cdot, \tilde{\varphi}_{k}^{\prime}(\cdot, \cdot), 0\right)\right)\right\|_{C^{0, \alpha}\left([0, T] \times \bar{C}_{k}\right)} .
$$

By using the local coordinate transforms $\left\{\psi_{k}\right\}_{k=1}^{m}$, we see that 5.16 is equivalent to

$$
\sup _{0<t<T}\left\|F^{u}\left(t, \psi_{k}\left(t, y^{\prime}, 0\right)\right)-F^{\tilde{u}}\left(t, \tilde{\psi}_{k}\left(t, w_{k}^{\prime}(t, y), 0\right)\right)\right\|_{C_{y}^{\alpha}(\bar{B})},
$$

where $w_{k}^{\prime}(t, y)$ is defined as

$$
\begin{aligned}
w_{k}^{\prime}(t, y) & :=\left(w_{k}^{(1)}(t, y), \ldots, w_{k}^{(n-1)}(t, y)\right), \\
w_{k}^{(i)}(t, y) & :=\tilde{\varphi}_{k}^{(i)}\left(t, \psi_{k}(t, y)\right) .
\end{aligned}
$$

In fact, we have the following proposition.

Proposition 5.1 Let $T_{0}$ be the positive number obtained in Proposition 6.1. Then for all $T \in$ $\left(0, T_{0}\right]$,

$$
\begin{aligned}
\sup _{0<t<T} \max _{1 \leqslant k \leqslant m}\left\|F^{u}\left(t, \psi_{k}\left(t, y^{\prime}, 0\right)\right)-F^{\tilde{u}}\left(t, \tilde{\psi}_{k}\left(t, w_{k}^{\prime}(t, y), 0\right)\right)\right\|_{C_{y}^{\alpha}(\bar{B})} \\
\quad \leqslant C T^{1 / 2}\left(1+\sup _{0<t<T} t^{1 / 2}\left\|\partial_{x}^{3} \tilde{v}(t, \cdot)\right\|_{C^{\alpha}\left(\bigcup_{k=1}^{m} \bar{O}_{k}\right)}\right)\|v-\tilde{v}\|_{C^{1,2+\alpha}\left([0, T] \times \bigcup_{k=1}^{m} \bar{O}_{k}\right)},
\end{aligned}
$$

where $C$ depends only on $n, \alpha, r$, and $R$.

The proof of the above proposition will be given in the Appendix; see Section 6.1. Here we only give a simple explanation why the term $\sup _{0<t<T} t^{1 / 2}\left\|\partial_{x}^{3} \tilde{v}(t, \cdot)\right\|_{C^{\alpha}\left(\bigcup_{k=1}^{m} \bar{O}_{k}\right)}$ appears in the above estimate. In order to establish the above estimate, we have to calculate the $C^{0, \alpha}$-norm of $H\left(t, \psi_{k}\left(t, y^{\prime}, 0\right)\right)-\tilde{H}\left(t, \tilde{\psi}_{k}\left(t, y^{\prime}, 0\right)\right)$ where $H:=H^{u}=-\frac{1}{n-1} \Delta v$ and $\tilde{H}:=H^{\tilde{u}}=-\frac{1}{n-1} \Delta \tilde{v}$. By decomposing this term as

$$
H\left(t, \psi_{k}\left(t, y^{\prime}, 0\right)\right)-\tilde{H}\left(t, \psi_{k}\left(t, y^{\prime}, 0\right)\right)+\tilde{H}\left(t, \psi_{k}\left(t, y^{\prime}, 0\right)\right)-\tilde{H}\left(t, \tilde{\psi}_{k}\left(t, y^{\prime}, 0\right)\right),
$$

we see that the term $\sup _{0<t<T} t^{1 / 2}\left\|\partial_{x}^{3} \tilde{v}(t, \cdot)\right\|_{C^{\alpha}\left(\bigcup_{k=1}^{m} \bar{O}_{k}\right)}$ appears when we estimate this term by the mean value theorem.

From the above proposition, we have

$$
\|\Psi(u)-\Psi(\tilde{u})\|_{C^{0, \alpha}\left([0, T] \times \mathbb{R}^{n}\right)} \leqslant \sigma_{1} C T^{1 / 2}\left(1+C_{4}\right)\|v-\tilde{v}\|_{C^{1,2+\alpha}([0, T] \times \bar{D})},
$$

where $C$ depends only on $n, \alpha, \Gamma_{0}$, and $M$. On the other hand, from Proposition 3.3 .

$$
\|v-\tilde{v}\|_{C^{1,2+\alpha}([0, T] \times \bar{D})} \leqslant C\|u-\tilde{u}\|_{C^{0, \alpha}\left([0, T] \times \mathbb{R}^{n}\right)},
$$

where $C$ depends only on $n, \alpha, \sigma_{2}, \Gamma_{0}$, and $M$. Collecting the above two estimates, we see that $\Psi$ is a contraction on $\mathcal{U}_{M}$ for sufficiently small $T$.

Therefore, $\Psi$ has a unique fixed point $u^{*} \in \mathcal{U}_{M}$. Since $u^{*}=\Psi\left(u^{*}\right)$, from the definition of $\Psi_{0}$, we have

$$
\Psi_{0}\left(u^{*}\right)(t, x)=F_{0}(t, x)+F^{u^{*}}(t, x)=F_{0}(t, x)+E^{v^{*}}\left(F^{u^{*}}\right)(t, x)=\Psi\left(u^{*}\right)(t, x)=u^{*}(t, x)
$$


for any $(t, x) \in \bigcup_{0 \leqslant t \leqslant T}\{t\} \times \Gamma_{t}^{u^{*}}$. Hence, $\left\{\Gamma_{t}^{u^{*}}\right\}_{0 \leqslant t \leqslant T}$ evolves by the equation

$$
\left\{\begin{array}{l}
\frac{\mathrm{d} x}{\mathrm{~d} t}=\sigma_{2} H^{*}(t, x) v^{*}(t, x)+u^{*}(t, x)=\sigma_{2} H^{*}(t, x) v^{*}(t, x)+\Psi_{0}\left(u^{*}\right)(t, x), \\
x(0)=x_{0} \in \Gamma_{0},
\end{array}\right.
$$

that is, the pair $\left(\Psi_{0}\left(u^{*}\right),\left\{\Gamma_{t}^{u^{*}}\right\}_{0 \leqslant t \leqslant T}\right)$ is a solution of our free boundary problem.

Although the above mapping $\Psi$ depends on the particular extension, we can show that the solution, in fact, does not, and is unique in the class stated in the main theorem. To see this, let $\left(u,\left\{\Gamma_{t}\right\}_{0 \leqslant t \leqslant T}\right)$ be another solution for $\left[\right.$ FBP . Let $v$ be the signed distance function of $\left\{\Gamma_{t}\right\}_{0 \leqslant t \leqslant T}$. Then $v \in C^{1,2+\alpha}([0, T] \times \bar{D})$ where $\bar{D}=\left\{x \in \mathbb{R}^{n} ;-\delta<d_{0}(x)<\delta\right\}$ for sufficiently small $\delta>0$. Since $\left\{\Gamma_{t}\right\}_{0 \leqslant t \leqslant T}$ evolves by the equation in $(\overline{\mathrm{BC}}, v$ satisfies $(3.6)$. An important fact is that for any $x \in \bar{D}$, the point $x-v(t, x) \nabla_{x} v(t, x)$ must belong to $\Gamma_{t}$ by the definition of the signed distance function. This implies that for any $\tilde{u}$ satisfying $\tilde{u}=u$ on $\bigcup_{0 \leqslant t \leqslant T}\{t\} \times \Gamma_{t}$, the function $v$ is also a solution of 3.6 with $\tilde{u}$ instead of $u$. This proves that the solution $\left(u,\left\{\Gamma_{t}\right\}_{0 \leqslant t \leqslant T}\right)$ does not depend on the particular extension, and the above $\left(\Psi_{0}\left(u^{*}\right),\left\{\Gamma_{t}^{u^{*}}\right\}_{0 \leqslant t \leqslant T}\right)$ is the unique solution solving $[\overline{\mathrm{FBP}}]$ in the class stated in the theorem. Now the proof of the main theorem is complete.

\section{Appendix}

\subsection{Proof of Proposition 5.1}

The proof is by direct calculation, essentially the same as in the proof of Proposition 4.1. For simplicity, we write $\|v-\tilde{v}\|_{C^{1,2+\alpha}},\left\|\partial_{x} \tilde{v}(t, \cdot)\right\|_{C^{2+\alpha}}$ instead of $\|v-\tilde{v}\|_{C^{1,2+\alpha}\left([0, T] \times \bigcup_{k=1}^{m} \bar{o}_{k}\right)}$, $\left\|\partial_{x} \tilde{v}(t, \cdot)\right\|_{C^{2+\alpha}\left(\bigcup_{k=1}^{m} \bar{o}_{k}\right)}$, respectively. We also write $w_{h}^{\prime}(y)$ instead of $w_{h}^{\prime}(t, y)$. First note that by (4.31, it suffices to estimate

$$
\begin{aligned}
& \mathcal{I}_{i, j, k}\left(t, s, \psi_{h}\left(t, y^{\prime}, 0\right)\right)-\tilde{\mathcal{I}}_{i, j, k}\left(t, s, \tilde{\psi}_{h}\left(t, w_{h}^{\prime}(y), 0\right)\right) \\
&=\int_{\mathbb{R}^{n-1}} K_{i, j}\left(t-s, \psi_{h}\left(t, y^{\prime}, 0\right)-\psi_{k}\left(s, \xi^{\prime}, 0\right)\right) \Upsilon_{1}\left(s, \xi^{\prime}\right) \mathrm{d} \xi^{\prime} \\
&-\int_{\mathbb{R}^{n-1}} K_{i, j}\left(t-s, \tilde{\psi}_{h}\left(t, w_{h}^{\prime}(y), 0\right)-\tilde{\psi}_{k}\left(s, \xi^{\prime}, 0\right)\right) \tilde{\Upsilon}_{1}\left(s, \xi^{\prime}\right) \mathrm{d} \xi^{\prime},
\end{aligned}
$$

where

$$
\begin{aligned}
& \Upsilon_{1}\left(s, \xi^{\prime}\right)=a_{k}\left(\psi_{k}\left(s, \xi^{\prime}, 0\right)\right)\left(H v_{j}\right)\left(s, \psi_{k}\left(s, \xi^{\prime}, 0\right)\right) J_{\psi_{k}}\left(s, \xi^{\prime}\right) \\
& \tilde{\Upsilon}_{1}\left(s, \xi^{\prime}\right)=a_{k}\left(\tilde{\psi}_{k}\left(s, \xi^{\prime}, 0\right)\right)\left(\tilde{H} \tilde{v}_{j}\right)\left(s, \tilde{\psi}_{k}\left(s, \xi^{\prime}, 0\right)\right) J_{\tilde{\psi}_{k}}\left(s, \xi^{\prime}\right)
\end{aligned}
$$

Set

$$
\begin{aligned}
& B_{1}:=\left\{y=\left(y^{\prime}, y^{(n)}\right) \in B ;\left(y^{\prime}, 0\right) \in B_{1}^{\prime}\right\}, \\
& B_{2}:=\left\{y=\left(y^{\prime}, y^{(n)}\right) \in B ;\left(y^{\prime}, 0\right) \in B_{2}^{\prime}\right\} .
\end{aligned}
$$

Here, $B_{1}^{\prime}$ and $B_{2}^{\prime}$ are the subsets of $B^{\prime}=\left\{\left(y^{\prime}, 0\right) ;\left|y^{\prime}\right|<1\right\}$ defined by 4.40 and 4.41 . We have $B=B_{1} \cup B_{2}$ and, as in the proof of Proposition 4.1 , for $f \in C(\bar{B})$,

$$
\|f\|_{C^{\alpha}(\bar{B})} \leqslant C\left(\|f\|_{C^{\alpha}\left(\bar{B}_{1}\right)}+\|f\|_{C^{\alpha}\left(\bar{B}_{2}\right)}\right),
$$

where $C$ depends only on $n, \delta_{1}, r$, and $R$. 
Hence we shall estimate

$$
\left\|\mathcal{I}_{i, j, k}\left(t, s, \psi_{h}\left(t, y^{\prime}, 0\right)\right)-\tilde{\mathcal{I}}_{i, j, k}\left(t, s, \tilde{\psi}_{h}\left(t, w_{h}^{\prime}(y), 0\right)\right)\right\|_{C_{y}^{\alpha}\left(\bar{B}_{1}\right)}
$$

and

$$
\left\|\mathcal{I}_{i, j, k}\left(t, s, \psi_{h}\left(t, y^{\prime}, 0\right)\right)-\tilde{\mathcal{I}}_{i, j, k}\left(t, s, \tilde{\psi}_{h}\left(t, w_{h}^{\prime}(y), 0\right)\right)\right\|_{C_{y}^{\alpha}\left(\bar{B}_{2}\right)} .
$$

If $y \in B_{1}$, then there exists a point $\left(\zeta^{\prime}, 0\right)=\left(\zeta^{\prime}\left(y^{\prime}\right), 0\right) \in B$ such that $\psi_{h}\left(t, y^{\prime}, 0\right)=\psi_{k}\left(t, \zeta^{\prime}, 0\right)$. In this case, since $\psi_{h}\left(t, y^{\prime}, 0\right) \in O_{k}$, we see that $\tilde{\psi}_{h}\left(t, w_{h}^{\prime}(y), 0\right) \in \tilde{\psi}_{k}(t, B)$ for all $t \in[0, T]$ if $\|v-\tilde{v}\|_{C^{1,2+\alpha}}$ is sufficiently small. Indeed, note that since $\psi_{h}(0, y)=\tilde{\psi}_{h}(0, y)$, by Proposition 6.1 in Section 6.2 below, we have

$$
\begin{aligned}
\left|\psi_{h}\left(t, y^{\prime}, 0\right)-\tilde{\psi}_{h}\left(t, w_{h}^{\prime}(y), 0\right)\right| & \leqslant\left|\psi_{h}\left(t, y^{\prime}, 0\right)-\tilde{\psi}_{h}\left(t, y^{\prime}, 0\right)\right|+\left|\tilde{\psi}_{h}\left(t, y^{\prime}, 0\right)-\tilde{\psi}_{h}\left(t, w_{h}^{\prime}(y), 0\right)\right| \\
& \leqslant\left\|\partial_{t} \psi_{h}-\partial_{t} \tilde{\psi}_{h}\right\|_{C([0, T] \times \bar{B})} T+\left\|\partial_{y} \tilde{\psi}_{h}\right\|_{C([0, T] \times \bar{B})}\left|y^{\prime}-w_{h}^{\prime}(y)\right| \\
& \leqslant C T\|v-\tilde{v}\|_{C^{1,2+\alpha}}+C\left|y^{\prime}-w_{h}^{\prime}(y)\right|
\end{aligned}
$$

where $C$ depends only on $n, r$, and $R$. We also have

$$
\begin{aligned}
\left|y^{\prime}-w_{h}^{\prime}(y)\right| & =\left|\varphi_{h}^{\prime}\left(t, \psi_{h}(t, y)\right)-\tilde{\varphi}_{h}^{\prime}\left(t, \psi_{h}(t, y)\right)\right| \leqslant\left\|\varphi_{h}(t, \cdot)-\tilde{\varphi}_{h}(t, \cdot)\right\|_{C\left(\bar{O}_{h}\right)} \\
& \leqslant T\left\|\partial_{t} \varphi_{h}-\partial_{t} \tilde{\varphi}_{h}\right\|_{C\left([0, T] \times \bar{O}_{h}\right)} \leqslant C T\|v-\tilde{v}\|_{C^{1,2+\alpha}} .
\end{aligned}
$$

Combining these with $O_{k} \subset \subset \bigcap_{0 \leqslant t \leqslant T} \tilde{\psi}_{k}(t, B)$, we obtain $\tilde{\psi}_{h}\left(t, w_{h}^{\prime}(y), 0\right) \in \tilde{\psi}_{k}(t, B)$ for $t \in$ $[0, T]$ if $\|v-\tilde{v}\|_{C^{1,2+\alpha}} \leqslant \epsilon_{1}, \epsilon_{1}$ is sufficiently small. Note that $\epsilon_{1}$ can be taken depending only on $n, r$, and $R$. We omit the details.

Since $\tilde{\psi}_{h}\left(t, w_{h}^{\prime}(y), 0\right) \in \tilde{\psi}_{k}(t, B)$, there exists a point $\left(\eta^{\prime}, 0\right)=\left(\eta^{\prime}(y), 0\right) \in B$ such that $\tilde{\psi}_{h}\left(t, w_{h}^{\prime}(y), 0\right)=\tilde{\psi}_{k}\left(t, \eta^{\prime}, 0\right)$. Thus, if $y \in B_{1}$, then we can decompose

$$
\mathcal{I}_{i, j, k}\left(t, s, \psi_{h}\left(t, y^{\prime}, 0\right)\right)-\tilde{\mathcal{I}}_{i, j, k}\left(t, s, \tilde{\psi}_{h}\left(t, w_{h}^{\prime}(y), 0\right)\right)=\mathcal{O}_{1}(t, s, y)+\mathcal{O}_{2}(t, s, y),
$$

where

$$
\begin{aligned}
\mathcal{O}_{1}(t, s, y): & =\mathcal{I}_{i, j, k}\left(t, s, \psi_{h}\left(t, y^{\prime}, 0\right)\right)-\tilde{\mathcal{I}}_{i, j, k}\left(t, s, \tilde{\psi}_{k}\left(t, \zeta^{\prime}\left(y^{\prime}\right), 0\right)\right) \\
& =\mathcal{I}_{i, j, k}\left(t, s, \psi_{k}\left(t, \zeta^{\prime}\left(y^{\prime}\right), 0\right)\right)-\tilde{\mathcal{I}}_{i, j, k}\left(t, s, \tilde{\psi}_{k}\left(t, \zeta^{\prime}\left(y^{\prime}\right), 0\right)\right), \\
\mathcal{O}_{2}(t, s, y) & :=\tilde{\mathcal{I}}_{i, j, k}\left(t, s, \tilde{\psi}_{k}\left(t, \zeta^{\prime}\left(y^{\prime}\right), 0\right)\right)-\tilde{\mathcal{I}}_{i, j, k}\left(t, s, \tilde{\psi}_{h}\left(t, w_{h}^{\prime}(y), 0\right)\right) \\
& =\tilde{\mathcal{I}}_{i, j, k}\left(t, s, \tilde{\psi}_{k}\left(t, \zeta^{\prime}\left(y^{\prime}\right), 0\right)\right)-\tilde{\mathcal{I}}_{i, j, k}\left(t, s, \tilde{\psi}_{k}\left(t, \eta^{\prime}(y), 0\right)\right) .
\end{aligned}
$$

We first estimate $\mathcal{O}_{1}$. By the definition of $\mathcal{I}_{i, j, k}$, the function $\mathcal{O}_{1}$ is expressed as

$$
\begin{aligned}
& \mathcal{O}_{1}(t, s, y)=\Upsilon_{7}(t, s, y)+\Upsilon_{8}(t, s, y), \\
& \Upsilon_{7}(t, s, y):=\int_{\mathbb{R}^{n-1}} K_{i, j}\left(t-s, \psi_{k}\left(t, \zeta^{\prime}\left(y^{\prime}\right), 0\right)-\psi_{k}\left(s, \xi^{\prime}, 0\right)\right)\left(\Upsilon_{1}-\tilde{\Upsilon}_{1}\right)\left(s, \xi^{\prime}\right) \mathrm{d} \xi^{\prime}, \\
& \Upsilon_{8}(t, s, y):=\int_{\mathbb{R}^{n-1}} \Upsilon_{9}\left(t, s, y, \xi^{\prime}\right) \tilde{\Upsilon}_{1}\left(s, \xi^{\prime}\right) \mathrm{d} \xi^{\prime},
\end{aligned}
$$


where

$\Upsilon_{9}\left(t, s, y, \xi^{\prime}\right)$

$$
\begin{aligned}
: & =K_{i, j}\left(t-s, \psi_{k}\left(t, \zeta^{\prime}\left(y^{\prime}\right), 0\right)-\psi_{k}\left(s, \xi^{\prime}, 0\right)\right)-K_{i, j}\left(t-s, \tilde{\psi}_{k}\left(t, \zeta^{\prime}\left(y^{\prime}\right)-\tilde{\psi}_{k}\left(s, \xi^{\prime}, 0\right), 0\right)\right. \\
& =\int_{0}^{1}\left\langle\Upsilon_{10}\left(t, s, y, \xi^{\prime}\right), \Upsilon_{11}\left(t, s, y, \xi^{\prime}, \tau_{1}\right)\right\rangle \mathrm{d} \tau_{1}
\end{aligned}
$$

with

$$
\begin{aligned}
\Upsilon_{10}\left(t, s, y, \xi^{\prime}\right) & :=\psi_{k}\left(t, \zeta^{\prime}\left(y^{\prime}\right), 0\right)-\psi_{k}\left(s, \xi^{\prime}, 0\right)-\tilde{\psi}_{k}\left(t, \zeta^{\prime}\left(y^{\prime}\right), 0\right)+\tilde{\psi}_{k}\left(s, \xi^{\prime}, 0\right), \\
\Upsilon_{11}\left(t, s, y, \xi^{\prime}, \tau_{1}\right) & :=\left(\nabla_{x} K_{i, j}\right)\left(t-s, \tau_{1} \Upsilon_{10}\left(t, s, y, \xi^{\prime}\right)+\tilde{\psi}_{k}\left(t, \zeta^{\prime}\left(y^{\prime}\right), 0\right)-\tilde{\psi}_{k}\left(s, \xi^{\prime}, 0\right)\right) .
\end{aligned}
$$

First we shall estimate $\Upsilon_{8}$. Using the mean value theorem, we see that

$$
\begin{aligned}
\left|\Upsilon_{10}\left(t, s, y, \xi^{\prime}\right)\right| \leqslant & \left|\psi_{k}\left(t, \zeta^{\prime}\left(y^{\prime}\right), 0\right)-\psi_{k}\left(s, \zeta^{\prime}\left(y^{\prime}\right), 0\right)-\left(\tilde{\psi}_{k}\left(t, \zeta^{\prime}\left(y^{\prime}\right), 0\right)-\tilde{\psi}_{k}\left(s, \zeta^{\prime}\left(y^{\prime}\right), 0\right)\right)\right| \\
& +\left|\psi_{k}\left(s, \zeta^{\prime}\left(y^{\prime}\right), 0\right)-\psi_{k}\left(s, \xi^{\prime}, 0\right)-\left(\tilde{\psi}_{k}\left(s, \zeta^{\prime}\left(y^{\prime}\right), 0\right)-\tilde{\psi}_{k}\left(s, \xi^{\prime}, 0\right)\right)\right| \\
\leqslant & \left\|\partial_{t} \psi_{k}-\partial_{t} \tilde{\psi}_{k}\right\|_{C([0, T] \times \bar{B})}(t-s)+\left\|\partial_{y} \psi_{k}-\partial_{y} \tilde{\psi}_{k}\right\|_{C([0, T] \times \bar{B})}\left|\zeta^{\prime}\left(y^{\prime}\right)-\xi^{\prime}\right| \\
\leqslant & C\|v-\tilde{v}\|_{C^{1,2+\alpha}}\left(t-s+T^{(1+\alpha) / 2}\left|\zeta^{\prime}\left(y^{\prime}\right)-\xi^{\prime}\right|\right),
\end{aligned}
$$

and for $y_{1}, y_{2} \in B_{1}$,

$$
\left|\Upsilon_{10}\left(t, s, y_{1}, \xi^{\prime}\right)-\Upsilon_{10}\left(t, s, y_{2}, \xi^{\prime}\right)\right| \leqslant C T^{(1+\alpha) / 2}\|v-\tilde{v}\|_{C^{1,2+\alpha}}\left|y_{1}-y_{2}\right| .
$$

From Lemma 4.1 and 4.25, we have

$$
\begin{aligned}
& \left|\Upsilon_{11}\left(t, s, y, \xi^{\prime}, \tau_{1}\right)\right| \\
& \quad \leqslant C(t-s)^{-(n+1) / 2}\left(1+\frac{\left|\tau_{1} \Upsilon_{10}\left(t, s, y, \xi^{\prime}\right)+\tilde{\psi}_{k}\left(t, \zeta^{\prime}\left(y^{\prime}\right), 0\right)-\tilde{\psi}_{k}\left(s, \xi^{\prime}, 0\right)\right|}{(t-s)^{1 / 2}}\right)^{-(n+1)} \\
& \quad \leqslant C(t-s)^{-(n+1) / 2}\left(1+\frac{r^{2}\left|\zeta^{\prime}\left(y^{\prime}\right)-\xi^{\prime}\right|}{64 R^{2}(t-s)^{1 / 2}}\right)^{-(n+1)} .
\end{aligned}
$$

For $y_{1}, y_{2} \in B_{1}$, it follows that

$$
\begin{aligned}
& \left|\Upsilon_{11}^{(l)}\left(t, s, y_{1}, \xi^{\prime}, \tau_{1}\right)-\Upsilon_{11}^{(l)}\left(t, s, y_{2}, \xi^{\prime}, \tau_{1}\right)\right| \\
& =\left|\int_{0}^{1}\left\langle\Upsilon_{12}\left(t, s, y_{1}, y_{2}, \xi^{\prime}, \tau_{1}\right), \Upsilon_{13}\left(t, s, y_{1}, y_{2}, \xi^{\prime}, \tau_{1}, \tau_{2}\right)\right\rangle \mathrm{d} \tau_{2}\right|,
\end{aligned}
$$

where

$$
\begin{aligned}
\Upsilon_{12}\left(t, s, y_{1}, y_{2}, \xi^{\prime}, \tau_{1}\right):= & \tau_{1} \Upsilon_{10}\left(t, s, y_{1}, \xi^{\prime}\right)+\tilde{\psi}_{k}\left(t, \zeta^{\prime}\left(y_{1}^{\prime}\right), 0\right)-\tilde{\psi}_{k}\left(s, \xi^{\prime}, 0\right) \\
& -\left(\tau_{1} \Upsilon_{10}\left(t, s, y_{2}, \xi^{\prime}\right)+\tilde{\psi}_{k}\left(t, \zeta^{\prime}\left(y_{2}^{\prime}\right), 0\right)-\tilde{\psi}_{k}\left(s, \xi^{\prime}, 0\right)\right) \\
= & \tau_{1}\left(\psi_{k}\left(t, \zeta^{\prime}\left(y_{1}^{\prime}\right), 0\right)-\psi_{k}\left(t, \zeta^{\prime}\left(y_{2}^{\prime}\right), 0\right)\right) \\
& +\left(1-\tau_{1}\right)\left(\tilde{\psi}_{k}\left(t, \zeta^{\prime}\left(y_{1}^{\prime}\right), 0\right)-\tilde{\psi}_{k}\left(t, \zeta^{\prime}\left(y_{2}^{\prime}\right), 0\right)\right), \\
\Upsilon_{13}\left(t, s, y_{1}, y_{2}, \xi^{\prime}, \tau_{1}, \tau_{2}\right):= & \left(\nabla_{x} \partial_{x_{l}} K_{i, j}\right)\left(t-s, \Upsilon_{14}\left(t, s, y_{1}, y_{2}, \xi^{\prime}, \tau_{1}, \tau_{2}\right)\right), \\
\Upsilon_{14}\left(t, s, y_{1}, y_{2}, \xi^{\prime}, \tau_{1}, \tau_{2}\right):= & \tau_{2} \Upsilon_{12}\left(t, s, y_{1}, y_{2}, \xi^{\prime}, \tau_{1}\right)+\tau_{1} \Upsilon_{10}\left(t, s, y_{2}, \xi^{\prime}\right) \\
& +\tilde{\psi}_{k}\left(t, \zeta^{\prime}\left(y_{2}^{\prime}\right), 0\right)-\tilde{\psi}_{k}\left(s, \xi^{\prime}, 0\right) .
\end{aligned}
$$


We have

$$
\left|\Upsilon_{12}\left(t, s, y_{1}, y_{2}, \xi^{\prime}, \tau_{1}\right)\right| \leqslant C\left|y_{1}-y_{2}\right|
$$

and from 4.25,

$$
\left|\Upsilon_{14}\left(t, s, y_{1}, y_{2}, \xi^{\prime}, \tau_{1}, \tau_{2}\right)\right| \geqslant \frac{r^{2}}{64 R^{2}}\left|\tau_{2} \zeta^{\prime}\left(y_{1}^{\prime}\right)+\left(1-\tau_{2}\right) \zeta^{\prime}\left(y_{2}^{\prime}\right)-\xi^{\prime}\right| .
$$

Thus, from Lemma 4.1. we have

$$
\begin{aligned}
& \left|\Upsilon_{11}^{(l)}\left(t, s, y_{1}, \xi^{\prime}, \tau_{1}\right)-\Upsilon_{11}^{(l)}\left(t, s, y_{2}, \xi^{\prime}, \tau_{1}\right)\right| \\
& \quad \leqslant C\left|y_{1}-y_{2}\right|(t-s)^{-n / 2-1} \int_{0}^{1}\left(1+\frac{\left|\Upsilon_{14}\left(t, s, y_{1}, y_{2}, \xi^{\prime}, \tau_{1}, \tau_{2}\right)\right|}{(t-s)^{1 / 2}}\right)^{-(n+2)} \mathrm{d} \tau_{2} \\
& \quad \leqslant C\left|y_{1}-y_{2}\right|(t-s)^{-n / 2-1} \int_{0}^{1}\left(1+\frac{r^{2}\left|\tau_{2} \zeta^{\prime}\left(y_{1}^{\prime}\right)+\left(1-\tau_{2}\right) \zeta^{\prime}\left(y_{2}^{\prime}\right)-\xi^{\prime}\right|}{64 R^{2}(t-s)^{1 / 2}}\right)^{-(n+2)} \mathrm{d} \tau_{2},
\end{aligned}
$$

where $C$ depends only on $n, r$, and $R$.

Collecting the above, we obtain

$\left|\Upsilon_{9}\left(t, s, y, \xi^{\prime}\right)\right|$

$$
\begin{aligned}
& \leqslant \int_{0}^{1}\left|\Upsilon_{10}\left(t, s, y, \xi^{\prime}\right)\right|\left|\Upsilon_{11}\left(t, s, y, \xi^{\prime}, \tau_{1}\right)\right| \mathrm{d} \tau_{1} \\
& \leqslant C\|v-\tilde{v}\|_{C^{1,2+\alpha}}\left(t-s+T^{(1+\alpha) / 2}\left|\zeta^{\prime}\left(y^{\prime}\right)-\xi^{\prime}\right|\right)(t-s)^{-(n+1) / 2}\left(1+\frac{r^{2}\left|\zeta^{\prime}\left(y^{\prime}\right)-\xi^{\prime}\right|}{64 R^{2}(t-s)^{1 / 2}}\right)^{-(n+1)}
\end{aligned}
$$

so

$$
\left\|\Upsilon_{8}(t, s, \cdot)\right\|_{C\left(\bar{B}_{1}\right)} \leqslant C\left(1+T^{(1+\alpha) / 2}(t-s)^{-1 / 2}\right)\|v-\tilde{v}\|_{C^{1,2+\alpha}},
$$

where $C$ depends only on $n, r$, and $R$. Moreover, for $y_{1}, y_{2} \in B_{1}$ with $\left|y_{1}-y_{2}\right| \leqslant(t-s)^{1 / 2}$,

$$
\begin{aligned}
&\left|\Upsilon_{9}\left(t, s, y_{1}, \xi^{\prime}\right)-\Upsilon_{9}\left(t, s, y_{2}, \xi^{\prime}\right)\right| \\
& \leqslant \int_{0}^{1}\left|\left\langle\Upsilon_{10}\left(t, s, y_{1}, \xi^{\prime}\right)-\Upsilon_{10}\left(t, s, y_{2}, \xi^{\prime}\right), \Upsilon_{11}\left(t, s, y_{1}, \xi^{\prime}, \tau_{1}\right)\right\rangle\right| \mathrm{d} \tau_{1} \\
&+\int_{0}^{1}\left|\left\langle\Upsilon_{10}\left(t, s, y_{2}, \xi^{\prime}\right), \Upsilon_{11}\left(t, s, y_{1}, \xi^{\prime}, \tau_{1}\right)-\Upsilon_{11}\left(t, s, y_{2}, \xi^{\prime}, \tau_{1}\right)\right\rangle\right| \mathrm{d} \tau_{1} \\
& \leqslant C T^{(1+\alpha) / 2}\|v-\tilde{v}\|_{C^{1,2+\alpha}}\left|y_{1}-y_{2}\right|(t-s)^{-(n+1) / 2}\left(1+\frac{r^{2}\left|\zeta^{\prime}\left(y_{1}^{\prime}\right)-\xi^{\prime}\right|}{64 R^{2}(t-s)^{1 / 2}}\right)^{-(n+1)} \\
&+C\|v-\tilde{v}\|_{C^{1,2+\alpha}}\left(t-s+T^{(1+\alpha) / 2}\left|\zeta^{\prime}\left(y_{2}^{\prime}\right)-\xi^{\prime}\right|\right)\left|y_{1}-y_{2}\right|(t-s)^{-n / 2-1} \\
& \times \int_{0}^{1}\left(1+\frac{r^{2}\left|\tau_{2} \zeta^{\prime}\left(y_{1}^{\prime}\right)+\left(1-\tau_{2}\right) \zeta^{\prime}\left(y_{2}^{\prime}\right)-\xi^{\prime}\right|}{64 R^{2}(t-s)^{1 / 2}}\right)^{-(n+2)} \mathrm{d} \tau_{2},
\end{aligned}
$$

and using the inequality

$$
\begin{aligned}
\left|\zeta^{\prime}\left(y_{2}^{\prime}\right)-\xi^{\prime}\right| & \leqslant\left|\tau_{2}\left(\zeta^{\prime}\left(y_{2}^{\prime}\right)-\zeta^{\prime}\left(y_{1}^{\prime}\right)\right)\right|+\left|\tau_{2} \zeta^{\prime}\left(y_{1}^{\prime}\right)+\left(1-\tau_{2}\right) \zeta^{\prime}\left(y_{2}^{\prime}\right)-\xi^{\prime}\right| \\
& \leqslant C\left|y_{1}-y_{2}\right|+\left|\tau_{2} \zeta^{\prime}\left(y_{1}^{\prime}\right)+\left(1-\tau_{2}\right) \zeta^{\prime}\left(y_{2}^{\prime}\right)-\xi^{\prime}\right| \\
& \leqslant C(t-s)^{1 / 2}+\left|\tau_{2} \zeta^{\prime}\left(y_{1}^{\prime}\right)+\left(1-\tau_{2}\right) \zeta^{\prime}\left(y_{2}^{\prime}\right)-\xi^{\prime}\right|
\end{aligned}
$$


we have

$$
\begin{aligned}
&\left|\Upsilon_{9}\left(t, s, y_{1}, \xi^{\prime}\right)-\Upsilon_{9}\left(t, s, y_{2}, \xi^{\prime}\right)\right| \\
& \leqslant C\|v-\tilde{v}\|_{C^{1,2+\alpha}}\left|y_{1}-y_{2}\right|\left\{T^{(1+\alpha) / 2}(t-s)^{-(n+1) / 2}\left(1+\frac{r^{2}\left|\zeta^{\prime}\left(y_{1}^{\prime}\right)-\xi^{\prime}\right|}{64 R^{2}(t-s)^{(1) / 2}}\right)^{-(n+1)}\right. \\
&+(t-s)^{-n / 2} \int_{0}^{1}\left(1+\frac{r^{2}\left|\tau_{2} \zeta^{\prime}\left(y_{1}^{\prime}\right)+\left(1-\tau_{2}\right) \zeta^{\prime}\left(y_{2}^{\prime}\right)-\xi^{\prime}\right|}{64 R^{2}(t-s)^{1 / 2}}\right)^{-(n+2)} \mathrm{d} \tau_{2} \\
&+T^{(1+\alpha) / 2}(t-s)^{-n / 2-1} \int_{0}^{1}\left(C(t-s)^{1 / 2}+\left|\tau_{2} \zeta^{\prime}\left(y_{1}^{\prime}\right)+\left(1-\tau_{2}\right) \zeta^{\prime}\left(y_{2}^{\prime}\right)-\xi^{\prime}\right|\right) \\
&\left.\cdot\left(1+\frac{r^{2}\left|\tau_{2} \zeta^{\prime}\left(y_{1}^{\prime}\right)+\left(1-\tau_{2}\right) \zeta^{\prime}\left(y_{2}^{\prime}\right)-\xi^{\prime}\right|}{64 R^{2}(t-s)^{1 / 2}}\right)^{-(n+2)} \mathrm{d} \tau_{2}\right\} .
\end{aligned}
$$

Hence using Fubini's theorem, we have for $y_{1}, y_{2} \in B_{1}$ with $\left|y_{1}-y_{2}\right| \leqslant(t-s)^{1 / 2}$,

$$
\begin{aligned}
\left|\Upsilon_{8}\left(t, s, y_{1}\right)-\Upsilon_{8}\left(t, s, y_{2}\right)\right| & \leqslant \int_{\mathbb{R}^{n-1}}\left|\Upsilon_{9}\left(t, s, y_{1}, \xi^{\prime}\right)-\Upsilon_{9}\left(t, s, y_{2}, \xi^{\prime}\right)\right|\left|\tilde{\Upsilon}_{1}\left(s, \xi^{\prime}\right)\right| \mathrm{d} \xi^{\prime} \\
& \leqslant C\|v-\tilde{v}\|_{C^{1,2+\alpha}\left|y_{1}-y_{2}\right|\left\{T^{(1+\alpha) / 2}(t-s)^{-1}+(t-s)^{-1 / 2}\right\},}
\end{aligned}
$$

where $C$ depends only on $n, r$, and $R$. On the other hand, for $y_{1}, y_{2} \in B_{1}$ with $\left|y_{1}-y_{2}\right|>(t-s)^{1 / 2}$, from 6.12) we have

$$
\begin{aligned}
\left|\Upsilon_{8}\left(t, s, y_{1}\right)-\Upsilon_{8}\left(t, s, y_{2}\right)\right| & \leqslant 2\left\|\Upsilon_{8}(t, s, \cdot)\right\|_{C\left(\bar{B}_{1}\right)} \\
& \leqslant C\|v-\tilde{v}\|_{C^{1,2+\alpha}}\left(1+T^{(1+\alpha) / 2}(t-s)^{-1 / 2}\right) \\
& \leqslant C\|v-\tilde{v}\|_{C^{1,2+\alpha}}\left((t-s)^{-1 / 2}+T^{(1+\alpha) / 2}(t-s)^{-1}\right)\left|y_{1}-y_{2}\right| .
\end{aligned}
$$

Thus for all $y_{1}, y_{2} \in B_{2}$, we obtain

$$
\left|\Upsilon_{8}\left(t, s, y_{1}\right)-\Upsilon_{8}\left(t, s, y_{2}\right)\right| \leqslant C\|v-\tilde{v}\|_{C^{1,2+\alpha}}\left((t-s)^{-1 / 2}+T^{(1+\alpha) / 2}(t-s)^{-1}\right)\left|y_{1}-y_{2}\right|,
$$

which implies that

$$
\left|\Upsilon_{8}\left(t, s, y_{1}\right)-\Upsilon_{8}\left(t, s, y_{2}\right)\right| \leqslant C\|v-\tilde{v}\|_{C^{1,2+\alpha}}\left((t-s)^{-\alpha / 2}+T^{(1+\alpha) / 2}(t-s)^{-(1+\alpha) / 2}\right)\left|y_{1}-y_{2}\right|^{\alpha},
$$

or

$$
\left[\Upsilon_{8}(t, s, \cdot)\right]_{C^{\alpha}\left(\bar{B}_{1}\right)} \leqslant C\|v-\tilde{v}\|_{C^{1,2+\alpha}}\left((t-s)^{-\alpha / 2}+T^{(1+\alpha) / 2}(t-s)^{-(1+\alpha) / 2}\right),
$$

where $C$ depends only on $n, r$, and $R$.

Next we shall estimate $\Upsilon_{7}$. By the definition of $\Upsilon_{1}, \tilde{\Upsilon}_{1}$, from Lemma 2.1.

$$
\left|\left(\Upsilon_{1}-\tilde{\Upsilon}_{1}\right)\left(s, \xi^{\prime}\right)\right| \leqslant C T^{\alpha / 2}\left(1+\sup _{0<t<T} t^{1 / 2}\left\|\partial_{x}^{3} \tilde{v}(t, \cdot)\right\|_{C^{\alpha}}\right)\|v-\tilde{v}\|_{C^{1,2+\alpha}},
$$

where $C$ depends only on $n, r$, and $R$. Thus together with the pointwise estimate in Lemma 4.1 and 4.23), we have

$$
\left\|\Upsilon_{7}(t, s, \cdot)\right\|_{C^{\alpha}\left(\bar{B}_{1}\right)} \leqslant C T^{\alpha / 2}(t-s)^{-1+\alpha / 2}\left(1+\sup _{0<t<T} t^{1 / 2}\left\|\partial_{x}^{3} \tilde{v}(t, \cdot)\right\|_{C^{\alpha}}\right)\|v-\tilde{v}\|_{C^{1,2+\alpha}},
$$

where $C$ depends only on $n, r$, and $R$. 
The estimate for $\mathcal{O}_{2}$ is also obtained by direct calculations as above:

$$
\begin{aligned}
& \left\|\mathcal{O}_{2}(t, s, \cdot)\right\|_{C^{\alpha}\left(\bar{B}_{1}\right)} \\
& \quad \leqslant C T^{(1+\alpha) / 2}(t-s)^{-1 / 2}\left(1+s^{-1 / 2} \sup _{0<t<T} t^{1 / 2}\left\|\partial_{x}^{3} \tilde{v}(t, \cdot)\right\| C^{\alpha}\right)\|v-\tilde{v}\|_{C^{1,2+\alpha}} .
\end{aligned}
$$

Next we shall estimate

$$
\left\|\mathcal{I}_{i, j, k}\left(t, s, \psi_{h}\left(t, y^{\prime}, 0\right)\right)-\tilde{\mathcal{I}}_{i, j, k}\left(t, s, \tilde{\psi}_{h}\left(t, w_{h}^{\prime}(y), 0\right)\right)\right\|_{C_{y}^{\alpha}\left(\bar{B}_{2}\right)} .
$$

Let $\epsilon_{2} \in\left(0, \epsilon_{1}\right]$ be a small positive number to be determined later. From 6.1) we decompose

$$
\mathcal{I}_{i, j, k}\left(t, s, \psi_{h}\left(t, y^{\prime}, 0\right)\right)-\tilde{\mathcal{I}}_{i, j, k}\left(t, s, \tilde{\psi}_{h}\left(t, w_{h}^{\prime}(y), 0\right)\right)=\mathcal{O}_{3}(t, s, y)+\mathcal{O}_{4}(t, s, y),
$$

where

$$
\begin{aligned}
\mathcal{O}_{3}(t, s, y):= & \int_{\mathbb{R}^{n-1}} K_{i, j}\left(t-s, \psi_{h}\left(t, y^{\prime}, 0\right)-\psi_{k}\left(s, \xi^{\prime}, 0\right)\right)\left(\Upsilon_{1}-\tilde{\Upsilon}_{1}\right)\left(s, \xi^{\prime}\right) \mathrm{d} \xi^{\prime}, \\
\mathcal{O}_{4}(t, s, y):= & \int_{\mathbb{R}^{n-1}}\left(K_{i, j}\left(t-s, \psi_{h}\left(t, y^{\prime}, 0\right)-\psi_{k}\left(s, \xi^{\prime}, 0\right)\right)\right. \\
& \left.-K_{i, j}\left(t-s, \tilde{\psi}_{h}\left(t, w_{h}^{\prime}(y), 0\right)-\tilde{\psi}_{k}\left(s, \xi^{\prime}, 0\right)\right)\right) \tilde{\Upsilon}_{1}\left(s, \xi^{\prime}\right) \mathrm{d} \xi^{\prime} .
\end{aligned}
$$

Let $\|v-\tilde{v}\|_{C^{1,2+\alpha}} \leqslant \epsilon_{2}$. Since $T \leqslant T_{0}<1$, if $\tilde{\psi}_{k}\left(s, \xi^{\prime}, 0\right) \in \operatorname{supp} a_{k}$ then for all $y \in B_{2}$,

$$
\begin{aligned}
\left|\psi_{h}\left(t, y^{\prime}, 0\right)-\psi_{k}\left(s, \xi^{\prime}, 0\right)\right| & \geqslant\left|\psi_{h}\left(t, y^{\prime}, 0\right)-\tilde{\psi}_{k}\left(s, \xi^{\prime}, 0\right)\right|-\left|\psi_{k}\left(s, \xi^{\prime}, 0\right)-\tilde{\psi}_{k}\left(s, \xi^{\prime}, 0\right)\right| \\
& \geqslant \delta_{1} / 2-C T\|v-\tilde{v}\|_{C^{1,2+\alpha}} \geqslant \delta_{1} / 2-C \epsilon_{2} \geqslant \delta_{1} / 4
\end{aligned}
$$

for sufficiently small $\epsilon_{2}$. Thus, from Lemma 4.1 and the estimate $(6.1)$, for all $y \in B_{2}$,

$$
\begin{aligned}
& \left|\mathcal{O}_{3}(t, s, y)\right| \\
& \quad \leqslant C \int_{B^{\prime}}(t-s)^{-n / 2}\left(1+\frac{\left|\psi_{h}\left(t, y^{\prime}, 0\right)-\psi_{k}\left(s, \xi^{\prime}, 0\right)\right|}{(t-s)^{1 / 2}}\right)^{-n}\left|\left(\Upsilon_{1}-\tilde{\Upsilon}_{1}\right)\left(s, \xi^{\prime}\right)\right| \mathrm{d} \xi^{\prime} \\
& \quad \leqslant C \int_{B^{\prime}}(t-s)^{-n / 2}\left(1+\frac{\delta_{1}}{4(t-s)^{1 / 2}}\right)^{-n} \mathrm{~d} \xi^{\prime}\left(1+s^{-1 / 2} \sup _{0<t<T} t^{1 / 2}\left\|\partial_{x}^{3} \tilde{v}(t, \cdot)\right\|_{C^{\alpha}}\right)\|v-\tilde{v}\|_{C^{1,2+\alpha}} \\
& \quad \leqslant C\left(1+s^{-1 / 2} \sup _{0<t<T} t^{1 / 2}\left\|\partial_{x}^{3} \tilde{v}(t, \cdot)\right\| C^{\alpha}\right)\|v-\tilde{v}\|_{C^{1,2+\alpha}},
\end{aligned}
$$

where $C$ depends only on $n, r$ and $R$.

For $y_{1}, y_{2} \in B_{2}$ with $\left|y_{1}-y_{2}\right| \leqslant \epsilon_{2}$, we see that for all $\xi^{\prime} \in B^{\prime}$ satisfying $\psi_{k}\left(s, \xi^{\prime}, 0\right) \in \operatorname{supp} a_{k}$ or $\tilde{\psi}_{k}\left(s, \xi^{\prime}, 0\right) \in \operatorname{supp} a_{k}$,

$$
\begin{aligned}
& \left|\tau_{1}\left(\psi_{h}\left(t, y_{1}^{\prime}, 0\right)-\psi_{k}\left(s, \xi^{\prime}, 0\right)\right)+\left(1-\tau_{1}\right)\left(\psi_{h}\left(t, y_{2}^{\prime}, 0\right)-\psi_{k}\left(s, \xi^{\prime}, 0\right)\right)\right| \\
& \quad \geqslant\left|\psi_{h}\left(t, y_{2}^{\prime}, 0\right)-\psi_{k}\left(s, \xi^{\prime}, 0\right)\right|-\left|\psi_{h}\left(t, y_{1}^{\prime}, 0\right)-\psi_{h}\left(t, y_{2}^{\prime}, 0\right)\right| \geqslant \delta_{1} / 4-C\left|y_{1}-y_{2}\right|,
\end{aligned}
$$

where $C$ depends on $n, r$, and $R$. Hence if $\epsilon_{2}$ is sufficiently small, then, for $y_{1}, y_{2} \in B_{2}$ with $\left|y_{1}-y_{2}\right| \leqslant \epsilon_{2}$, 


$$
\begin{aligned}
\left|\mathcal{O}_{3}\left(t, s, y_{1}\right)-\mathcal{O}_{3}\left(t, s, y_{2}\right)\right| & \mid \int_{B^{\prime}} \int_{0}^{1}\left\langle\psi_{h}\left(t, y_{1}^{\prime}, 0\right)-\psi_{h}\left(t, y_{2}^{\prime}, 0\right),\right. \\
& \left.\left(\nabla_{x} K_{i, j}\right)\left(t-s, \tau_{1} \psi_{h}\left(t, y_{1}^{\prime}, 0\right)+\left(1-\tau_{1}\right) \psi_{h}\left(t, y_{2}^{\prime}, 0\right)-\psi_{k}\left(s, \xi^{\prime}, 0\right)\right)\right\rangle \mathrm{d} \tau_{1}\left(\Upsilon_{1}-\tilde{\Upsilon}_{1}\right)\left(s, \xi^{\prime}\right) \mathrm{d} \xi^{\prime} \mid \\
\leqslant & C\left|y_{1}-y_{2}\right|(t-s)^{-(n+1) / 2} \\
& \times\left(1+\frac{\delta_{1}}{8(t-s)^{1 / 2}}\right)^{-(n+1)}\left(1+s^{-1 / 2} \sup _{0<t<T} t^{1 / 2}\left\|\partial_{x}^{3} \tilde{v}(t, \cdot)\right\|_{C^{\alpha}}\right)\|v-\tilde{v}\|_{C^{1,2+\alpha}} \\
\leqslant & C\left|y_{1}-y_{2}\right|\left(1+s^{-1 / 2} \sup _{0<t<T} t^{1 / 2}\left\|\partial_{x}^{3} \tilde{v}(t, \cdot)\right\| C^{\alpha}\right)\|v-\tilde{v}\|_{C^{1,2+\alpha}},
\end{aligned}
$$

where $C$ depends on $n, r$, and $R$. If $\left|y_{1}-y_{2}\right|>\epsilon_{2}$, then

$$
\begin{aligned}
\left|\mathcal{O}_{3}\left(t, s, y_{1}\right)-\mathcal{O}_{3}\left(t, s, y_{2}\right)\right| & \leqslant 2\left\|\mathcal{O}_{3}(t, s, \cdot)\right\|_{C\left(\bar{B}_{2}\right)} \\
& \leqslant C\left(1+s^{-1 / 2} \sup _{0<t<T} t^{1 / 2}\left\|\partial_{x}^{3} \tilde{v}(t, \cdot)\right\| C^{\alpha}\right)\|v-\tilde{v}\|_{C^{1,2+\alpha}} \\
& \leqslant C\left(1+s^{-1 / 2} \sup _{0<t<T} t^{1 / 2}\left\|\partial_{x}^{3} \tilde{v}(t, \cdot)\right\| C^{\alpha}\right)\|v-\tilde{v}\|_{C^{1,2+\alpha}}\left|y_{1}-y_{2}\right| / \epsilon_{2}
\end{aligned}
$$

where $C$ depends on $n, r$, and $R$. Similarly, if $\|v-\tilde{v}\|_{C^{1,2+\alpha}} \leqslant \epsilon_{2}$, it is not difficult to see that

$$
\left\|\mathcal{O}_{4}(t, s, \cdot)\right\|_{C^{\alpha}\left(\bar{B}_{2}\right)} \leqslant C\|v-\tilde{v}\|_{C^{1,2+\alpha}},
$$

where $C$ depends on $n, r$, and $R$.

Collecting these, if $\|v-\tilde{v}\|_{C^{1,2+\alpha}} \leqslant \epsilon_{2}$, we have

$$
\begin{aligned}
& \left\|\mathcal{I}_{i, j, k}\left(t, s, \psi_{h}\left(t, y^{\prime}, 0\right)\right)-\tilde{\mathcal{I}}_{i, j, k}\left(t, s, \tilde{\psi}_{h}\left(t, w_{h}^{\prime}(y), 0\right)\right)\right\|_{C_{y}^{\alpha}\left(\bar{B}_{2}\right)} \\
& \quad \leqslant C\left(1+s^{-1 / 2} \sup _{0<t<T} t^{1 / 2}\left\|\partial_{x}^{3} \tilde{v}(t, \cdot)\right\|_{C^{\alpha}}\right)\|v-\tilde{v}\|_{C^{1,2+\alpha}}
\end{aligned}
$$

where $C$ depends on $n, r$, and $R$. Note that $\epsilon_{2}$ depends only on $n, r$, and $R$. By Proposition 4.1, we easily see that

$$
\left\|\mathcal{I}_{i, j, k}\left(t, s, \psi_{h}\left(t, y^{\prime}, 0\right)\right)\right\|_{C_{y}^{\alpha}\left(\bar{B}_{2}\right)}+\left\|\tilde{\mathcal{I}}_{i, j, k}\left(t, s, \tilde{\psi}_{h}\left(t, w_{h}^{\prime}(y), 0\right)\right)\right\|_{C_{y}^{\alpha}\left(\bar{B}_{2}\right)} \leqslant C,
$$

where $C$ depends on $n, r$, and $R$. Hence, the estimate 6.15 holds for all $v, \tilde{v}$ with a constant $C$ depending only on $n, r$, and $R$. This completes the proof.

\subsection{Implicit function theorem}

Let $\Gamma_{0}$ be the boundary of a smooth bounded domain $\Omega_{0}$. Let $d_{0}$ be the signed distance function and we set

$$
D=\left\{x \in \mathbb{R}^{n} ;-\delta_{0}<d_{0}(x)<\delta_{0}\right\} .
$$

In order to estimate the term

$$
\int_{0}^{t} e^{(t-s) \Delta} \mathbf{P} H \nu \mathcal{H}_{\left\llcorner\Gamma_{s}\right.}^{n-1} \mathrm{~d} s,
$$


we need some information on the local coordinate transforms $\left\{\varphi_{j}\right\}$ associated with $\left\{\Gamma_{t}\right\}_{0 \leqslant t \leqslant T}$ belonging to $\mathcal{S}\left(\alpha, R, T, d_{0}\right)$. Let $r$ be the number given by 4.11 . For $A \subset \mathbb{R}^{n}$ and $\rho>0$, we set

$$
(A)_{\rho}:=\left\{x \in \mathbb{R}^{n} ; \operatorname{dist}(x, A)<\rho\right\} .
$$

Proposition 6.1 Let $R \geqslant 1$ and $\alpha \in(0,1)$. Then there exists a positive $T_{0}$ depending only on $r$ and $R$ such that for all $T \in\left(0, T_{0}\right]$ and $\left\{\Gamma_{t}\right\}_{0 \leqslant t \leqslant T} \in \mathcal{S}\left(\alpha, R, T, d_{0}\right)$, the following holds. There exist a family of open sets $\left\{U_{j}\right\}_{j=1}^{m}, U_{j} \subset \bar{D}$, and a family of functions $\left\{\varphi_{j}(t, x)\right\}_{j=1}^{m}, \varphi_{j}(t, x) \in$ $C^{1,2+\alpha}\left([0, T] \times \bar{U}_{j}\right)$, satisfying the following conditions:

(i) For each $t \in[0, T]$, there exists an open set $U_{j}(t) \subset U_{j}$ such that:

(i1) The functions $\varphi_{j}(t, \cdot): \overline{U_{j}(t)} \rightarrow \bar{B}\left(B:=\left\{y \in \mathbb{R}^{n} ;|y|<1\right\}\right)$ are $C^{2}$-diffeomorphisms.

(i2) For each $t \in[0, T]$,

$$
\begin{aligned}
\varphi_{j}\left(t,\left\{\overline{U_{j}(t)} \cap \Omega_{t}\right\}\right) & =\left\{y \in \bar{B} ; y_{n}>0\right\} \\
\varphi_{j}\left(t,\left\{\overline{U_{j}(t)} \cap \Gamma_{t}\right\}\right) & =\overline{B^{\prime}} \quad\left(B^{\prime}:=\left\{y \in B ; y_{n}=0\right\} .\right.
\end{aligned}
$$

(i3) For some $\rho>0$, there exist families of open balls $\left\{O_{j}\right\}_{j=1}^{m}$ and $\left\{\hat{O}_{j}\right\}_{j=1}^{m}$ such that

$$
\begin{aligned}
& \hat{O}_{j} \subset \subset O_{j}, \\
& O_{j} \subset \subset \bigcap_{0 \leqslant t \leqslant T} \varphi_{j}^{-1}(t, B), \quad 1 \leqslant j \leqslant m, \\
& \bigcup_{0 \leqslant t \leqslant T}\left(\Gamma_{t}\right)_{\rho} \subset \subset \bigcup_{j=1}^{m} \hat{O}_{j} .
\end{aligned}
$$

The above $\rho,\left\{U_{j}\right\}_{j=1}^{m},\left\{O_{j}\right\}_{j=1}^{m}$, and $\left\{\hat{O}_{j}\right\}_{j=1}^{m}$ are taken independently with respect to each evolving hypersurface belonging to $\bigcup_{0<T \leqslant T_{0}} \mathcal{S}\left(\alpha, R, T, d_{0}\right)$. In particular, we can take $\rho=$ $\frac{r^{2}}{(32 R)^{2}(1+R / r)}, \hat{O}_{j}=\left\{\left|x-\bar{x}_{j}\right|<3 \rho\right\}$, and $O_{j}=\left\{\left|x-\bar{x}_{j}\right|<4 \rho\right\}$ for some $\bar{x}_{j} \in \Gamma_{0}$.

(ii) Set $\psi_{j}(t, y):=\varphi_{j}^{-1}(t, y): \bar{B} \rightarrow \overline{U_{j}(t)}$. Then there exists a positive constant $C$ depending only on $n, r$, and $R$ such that

$$
\left\|\varphi_{j}\right\|_{C^{1,2+\alpha}\left([0, T] \times \bar{U}_{j}\right)},\left\|\psi_{j}\right\|_{C^{1,2+\alpha}([0, T] \times \bar{B})} \leqslant C\left(1+\|v\|_{C^{1,2+\alpha}([0, T] \times \bar{D})}\right) .
$$

Proof. The assertions essentially follow from the implicit function theorem. However, we shall give the proof for the convenience to the reader.

For any $\bar{x} \in \Gamma_{0}$, there exists $i_{0} \in\{1, \ldots, n\}$ such that

$$
\max _{1 \leqslant i \leqslant n}\left|\partial_{i} v(0, \bar{x})\right|=\left|\partial_{i_{0}} v(0, \bar{x})\right| \geqslant r .
$$

Without loss of generality we may assume that $i_{0}=n$ and $\partial_{n} v(0, \bar{x}) \geqslant r>0$. We set $\eta:=r^{2} / 32 R$ and $V_{\bar{x}}:=\left\{\left|x^{\prime}-\bar{x}^{\prime}\right|<\eta\right\}, W_{\bar{x}^{(n)}}:=\left\{\left|x^{(n)}-\bar{x}^{(n)}\right|<4 \eta / r\right\}$. Then it is easy to see that $U_{\bar{x}}:=$ $V_{\bar{x}^{\prime}} \times W_{\bar{x}^{(n)}} \subset D$ and

$$
\partial_{n} v(t, x) \geqslant r / 2, \quad(t, x) \in[0, T] \times \bar{U}_{\bar{x}},
$$


if $T$ is sufficiently small. Now consider the function of $x^{(n)}$ defined as $v^{\left(0, \bar{x}^{\prime}\right)}\left(x^{(n)}\right):=v\left(0, \bar{x}^{\prime}, x^{(n)}\right)$. Then $v^{\left(0, \bar{x}^{\prime}\right)}$ is strictly increasing and $v^{\left(0, \bar{x}^{\prime}\right)}\left(\bar{x}^{(n)}\right)=0$. Thus, for $y \in \bar{W}_{\bar{x}^{(n)}}$,

$$
\begin{aligned}
& y>\bar{x}^{(n)} \Leftrightarrow v\left(0, \bar{x}^{\prime}, y\right)=v^{\left(0, \bar{x}^{\prime}\right)}(y)>0 . \\
& y<\bar{x}^{(n)} \Leftrightarrow v\left(0, \bar{x}^{\prime}, y\right)=v^{\left(0, \bar{x}^{\prime}\right)}(y)<0 .
\end{aligned}
$$

In particular,

$$
\alpha_{1}:=v\left(0, \bar{x}^{\prime}, \bar{x}^{(n)}+4 \eta / r\right)>0>v\left(0, \bar{x}^{\prime}, \bar{x}^{(n)}-4 \eta / r\right)=: \alpha_{2} .
$$

Each $\left|\alpha_{i}\right|$ is estimated from below as $\left|\alpha_{i}\right| \geqslant 4 \eta$. Indeed,

$$
\begin{aligned}
v\left(0, \bar{x}^{\prime}, \bar{x}^{(n)}+4 \eta / r\right) & =v\left(0, \bar{x}^{\prime}, \bar{x}^{(n)}+4 \eta / r\right)-v\left(0, \bar{x}^{\prime}, \bar{x}^{(n)}\right) \\
& =\int_{0}^{4 \eta / r} \partial_{n} v\left(0, \bar{x}^{\prime}, \bar{x}^{(n)}+s\right) \mathrm{d} s \geqslant 4 \eta .
\end{aligned}
$$

The estimate for $\alpha_{2}$ is similarly obtained. From this, we can see that, for $\left(t, x^{\prime}\right) \in[0, T] \times \bar{V}_{\bar{x}^{\prime}}$,

$$
v\left(t, x^{\prime}, \bar{x}^{(n)}+4 \eta / r\right) \geqslant 2 \eta>0>-2 \eta \geqslant v\left(t, x^{\prime}, \bar{x}^{(n)}-4 \eta / r\right),
$$

for sufficiently small $T$.

Since the function $v^{\left(t, x^{\prime}\right)}\left(x^{(n)}\right):=v\left(t, x^{\prime}, \bar{x}^{(n)}\right)$ is also strictly increasing on $\bar{W}_{\bar{x}^{(n)}}$, for any $\left(t, x^{\prime}\right) \in[0, T] \times \bar{V}_{\bar{x}^{\prime}}$ there exists a unique $x^{(n)} \in \bar{W}_{\bar{x}^{(n)}}$ such that

$$
v\left(t, x^{\prime}, x^{(n)}\right)=0 .
$$

We write this correspodence as $x^{(n)}=g_{\bar{x}}\left(t, x^{\prime}\right)$. Note that

$$
\begin{aligned}
& y>x^{(n)} \Leftrightarrow v\left(t, x^{\prime}, y\right)>0, \\
& y<x^{(n)} \Leftrightarrow v\left(t, x^{\prime}, y\right)<0 .
\end{aligned}
$$

By definition, $\bar{x}^{(n)}=g_{\bar{x}}\left(0, x^{\prime}\right)$ and $v\left(t, x^{\prime}, g_{\bar{x}}\left(t, x^{\prime}\right)\right)=0$ for $\left(t, x^{\prime}\right) \in[0, T] \times \bar{V}_{\bar{x}^{\prime}}$. Conversely, if $\left(t, x^{\prime}, x^{(n)}\right) \in[0, T] \times \bar{V}_{\bar{x}^{\prime}} \times \bar{W}_{\bar{x}^{(n)}}$ and $v\left(t, x^{\prime}, x^{(n)}\right)=0$, then since $v^{\left(t, x^{\prime}\right)}\left(x^{(n)}\right)$ is strictly increasing on $\bar{W}_{\bar{x}^{(n)}}$, we must have $x^{(n)}=g_{\bar{x}}\left(t, x^{\prime}\right)$. It is not difficult to check that $g_{\bar{x}}\left(t, x^{\prime}\right) \in$ $C^{1,2+\alpha}\left([0, T] \times \bar{U}_{\bar{x}}\right)$. In fact, the first derivatives of $g_{\bar{x}}$ are given by

$$
\begin{aligned}
& \partial_{t} g_{\bar{x}}\left(t, x^{\prime}\right)=-\frac{\partial_{t} v\left(t, x^{\prime}, g_{\bar{x}}\left(t, x^{\prime}\right)\right)}{\partial_{n} v\left(t, x^{\prime}, g_{\bar{x}}\left(t, x^{\prime}\right)\right)}, \\
& \partial_{i} g_{\bar{x}}\left(t, x^{\prime}\right)=-\frac{\partial_{i} v\left(t, x^{\prime}, g_{\bar{x}}\left(t, x^{\prime}\right)\right)}{\partial_{n} v\left(t, x^{\prime}, g_{\bar{x}}\left(t, x^{\prime}\right)\right)}, \quad 1 \leqslant i \leqslant n-1,
\end{aligned}
$$

and these are estimated as

$$
\left\|\partial_{t} g\right\|_{C\left([0, T] \times \bar{V}_{\bar{x}_{j}}\right)},\left\|\partial_{x} g\right\|_{C\left([0, T] \times \bar{V}_{\bar{x}_{j}}\right)} \leqslant 2 R / r .
$$

Let $N \geqslant 1$ be a sufficiently large number to be determined later. Since $\Gamma_{0}$ is compact, there exists a sequence $\left\{\bar{x}_{j}\right\}_{j=1}^{m} \subset \Gamma_{0}$ such that

$$
\Gamma_{0} \subset \bigcup_{j=1}^{m}\left\{\left|x-\bar{x}_{j}\right|<\eta / 2 N\right\} \subset \bigcup_{j=1}^{m} U_{\bar{x}_{j}} .
$$


Moreover, there exists a positive number $\rho>0$ such that

$$
\left(\Gamma_{0}\right)_{\rho} \subset \bigcup_{j=1}^{m}\left\{\left|x-\bar{x}_{j}\right|<3 \eta / 2 N\right\},
$$

where $\left(\Gamma_{0}\right)_{\rho}=\left\{x \in D ; \operatorname{dist}\left(x, \Gamma_{0}\right)<\rho\right\}$.

In fact, we can take $\rho=\eta / N$. To see this, we take any $x \in\left(\Gamma_{0}\right)_{\rho}$, for this $\rho$. Then there exists a point $z \in \Gamma_{0}$ such that

$$
|x-z|=\operatorname{dist}\left(x, \Gamma_{0}\right) .
$$

Since $\Gamma_{0} \subset \bigcup_{j=1}^{m}\left\{\left|x-\bar{x}_{j}\right|<\eta / 2 N\right\}$, we have $z \in\left\{\left|x-\bar{x}_{j}\right|<\eta / 2 N\right\}$ for some $j$. Hence

$$
\left|x-\bar{x}_{j}\right| \leqslant|x-z|+\left|z-\bar{x}_{j}\right|<\frac{3 \eta}{2 N},
$$

and the claim follows.

We also have, for sufficiently small $T$,

$$
\bigcup_{0 \leqslant t \leqslant T} \Gamma_{t} \subset\left(\Gamma_{0}\right)_{\rho}
$$

To see this, note that since $\overline{D \backslash\left(\Gamma_{0}\right)_{\rho}}$ is compact, we have

$$
r^{\prime}:=\min \left\{|v(0, x)| ; x \in \overline{D \backslash\left(\Gamma_{0}\right)_{\rho}}\right\}>0 .
$$

So it follows that

$$
\left\{x \in \bar{D} ;|v(0, x)|<r^{\prime}\right\} \subset\left(\Gamma_{0}\right)_{\rho} .
$$

Since each $x \in \Gamma_{t}$ satisfies $v(t, x)=0$, we have

$$
|v(0, x)|=|v(0, x)-v(t, x)| \leqslant R T<r^{\prime}
$$

if $T$ is sufficiently small. This proves that $\bigcup_{0 \leqslant t \leqslant T} \Gamma_{t} \subset\left(\Gamma_{0}\right)_{\rho}$.

Next we shall show that for all $t \in(0, T]$,

$$
\left(\Gamma_{t}\right)_{\rho} \subset \subset \bigcup_{j=1}^{m} \hat{O}_{j}
$$

where $\hat{O}_{j}:=\left\{\left|x-\bar{x}_{j}\right|<3 \eta / N\right\}$. Indeed, for $x \in\left(\Gamma_{t}\right)_{\rho}$, there exists $z \in \Gamma_{t}$ such that $|x-z|=$ $\operatorname{dist}\left(x, \Gamma_{t}\right)<\rho$. Since $\Gamma_{t} \subset\left(\Gamma_{0}\right)_{\rho} \subset \bigcup_{j=1}^{m}\left\{\left|x-\bar{x}_{j}\right|<3 \eta / 2 N\right\}$, for some $j$ we have

$$
\left|x-\bar{x}_{j}\right| \leqslant|x-z|+\left|z-\bar{x}_{j}\right|<\frac{5 \eta}{2 N}
$$

which shows the above claim.

Now let $\psi_{j}(t, y)=\left(\psi_{j}^{(1)}(t, y), \ldots, \psi_{j}^{(n)}(t, y)\right)$ be a function on $[0, T] \times \bar{B}$ defined as follows:

$$
\begin{aligned}
\psi_{j}^{(i)}(t, y) & :=\frac{\eta}{2 R} y^{(i)}+\bar{x}_{j}^{(i)}, \quad 1 \leqslant i \leqslant n-1, \\
\psi_{j}^{(n)}(t, y) & :=\frac{\eta}{2 R} y^{(n)}+g_{\bar{x}_{j}}\left(t, \frac{\eta}{2 R} y^{\prime}+\bar{x}_{j}^{\prime}\right) .
\end{aligned}
$$


Note that since $\frac{\eta}{2 R} y^{\prime}+\bar{x}_{j}^{\prime} \in V_{\bar{x}_{j}^{\prime}}$ for $\left|y^{\prime}\right| \leqslant 1, \psi_{j}$ is well-defined. From now on, we write $g_{j}$ instead of $g_{\bar{x}_{j}}$. Since we have

$$
\begin{aligned}
\left|\psi_{j}^{(n)}(t, y)-\bar{x}_{j}^{(n)}\right| & \leqslant \frac{\eta}{2 R}\left|y^{(n)}\right|+\left|g_{j}\left(t, \frac{\eta}{2 R} y^{\prime}+\bar{x}_{j}^{\prime}\right)-g_{j}\left(0, \bar{x}_{j}^{\prime}\right)\right| \\
& \leqslant \frac{\eta}{2 R}+\left\|\partial_{x} g_{j}\right\|_{C\left([0, T] \times \bar{V}_{\bar{V}_{j}}\right)} \frac{\eta}{2 R}+T\left\|\partial_{t} g_{j}\right\|_{C\left([0, T] \times \bar{V}_{\bar{x}_{j}}\right)} \\
& \leqslant \frac{\eta}{2 R}+\frac{2 R}{r}\left(\frac{\eta}{2 R}+T\right),
\end{aligned}
$$

if we take $T \leqslant \eta / 2 R$, then $\psi_{j}^{(n)}(t, y) \in W_{\bar{x}_{j}^{(n)}}$ for all $y \in B$. Thus $\psi_{j}(t, B) \subset U_{\bar{x}_{j}}$ and we have the inverse function of $\psi_{j}$ given by

$$
\begin{aligned}
\varphi_{j}^{(i)}(t, x) & :=\frac{2 R}{\eta}\left(x^{(i)}-\bar{x}_{j}^{(i)}\right), \quad 1 \leqslant i \leqslant n-1, \\
\varphi_{j}^{(n)}(t, x) & :=\frac{2 R}{\eta}\left(x^{(n)}-g_{j}\left(t, x^{\prime}\right)\right) .
\end{aligned}
$$

Note that $\varphi_{j}$ can be defined on $[0, T] \times \bar{U}_{\bar{x}_{j}}$. Obviously $\varphi_{j}: \overline{U_{j}(t)}:=\psi_{j}(t, \bar{B}) \rightarrow \bar{B}$ is a $C^{2}$-diffeomorphism. Now we claim that if $N$ is sufficiently large and $T$ is sufficiently small, then $O_{j}:=\left\{x ;\left|x-\bar{x}_{j}\right|<4 \eta / N\right\} \subset \subset\left\{x ;\left|\varphi_{j}(t, x)\right| \leqslant 1\right\}\left(=\psi_{j}(t, \bar{B})\right)$ for all $t \in[0, T]$. Indeed, if $x \in O_{j}$, then

$$
\begin{aligned}
\left|\varphi_{j}(t, x)\right| & \leqslant \frac{2 R}{\eta}\left|x^{\prime}-\bar{x}_{j}^{\prime}\right|+\frac{2 R}{\eta}\left|x^{(n)}-g_{j}\left(t, x^{\prime}\right)\right| \\
& <\frac{2 R}{\eta} \cdot \frac{4 \eta}{N}+\frac{2 R}{\eta}\left(\left|x^{(n)}-\bar{x}_{j}^{(n)}\right|+\left|\bar{x}_{j}^{(n)}-g_{j}\left(t, \bar{x}_{j}^{\prime}\right)\right|+\left|g_{j}\left(t, \bar{x}_{j}^{\prime}\right)-g_{j}\left(t, x^{\prime}\right)\right|\right) \\
& \leqslant \frac{16 R}{N}+\frac{8 R\left\|\partial_{x} g_{j}\right\|_{C\left([0, T] \times \bar{V}_{\bar{x}_{j}^{\prime}}\right)}}{N}+\frac{2 R T\left\|\partial_{t} g_{j}\right\|_{C\left([0, T] \times \bar{V}_{\bar{x}_{j}^{(n)}}\right)}}{\eta} \\
& \leqslant \frac{16 R}{N}\left(1+\frac{R}{r}\right)+\frac{4 R^{2} T}{r \eta},
\end{aligned}
$$

which proves the claim with $N=32 R(1+R / r)$ and small $T$. Combining the above, we see that Proposition 6.1 i) holds. The estimates for $\varphi_{j}$ and $\psi_{j}$ follow from 66.21, 6.22), and 6.26]-66.29].

\section{Acknowledgements}

The author is grateful to Professor Chun Liu for suggesting the problem considered in this paper. He would like to express his deep gratitude to Professors Yoshikazu Giga, Yoshihiro Tonegawa, and Shin'ya Matsui for their helpful comments and advice on this problem. The author is grateful to Professor Helmut Abels for commenting on the contents of the paper during his stays in Hokkaido University. The author would also like to thank the anonymous referee for many helpful comments, especially on the background of our model. 


\section{REFERENCES}

1. Abels, H. On generalized solutions of two phase flows for viscous incompressible fluids. Interfaces Free Bound. 9 (2007), 31-65. Zbl 05174078 MR 2317298

2. Blesgen, T. A generalization of the Navier-Stokes equations to two-phase flows. J. Phys. D (Appl. Phys.) 32 (1999), 1119-1123. Zbl 0925.35120

3. Chang, Y. C., Hou, T. Y., Merriman, B., \& Osher, S. A level set formulation of Eulerian interface capturing methods for incompressible fluid flows. J. Comput. Phys. 124 (1996), 449-464. Zbl 0847.76048 MR 1383769

4. Denisova, I. V. Problem of the motion of two viscous incompressible fluids separated by a closed free interface. Acta Appl. Math. 37 (1994), 31-40. Zbl 0814.35093 MR 1308743

5. Evans, L. C., \& Spruck, J. Motion of level sets by mean curvature. II. Trans. Amer. Math. Soc. 330 (1992), 321-332. Zbl 0776.53005 MR 1068927

6. Giga, Y., \& Goto, S. Geometric evolution of phase-boundaries. On the Evolution of Phase Boundaries (Minneapolis, MN, 1990-91), IMA Vol. Math. Appl. 43, Springer, New York (1992), 51-65. Zbl 0771.35027 MR 1226914

7. Giga, Y., \& TAKAhASHI, S. On global weak solutions of the nonstationary two-phase Stokes flow. SIAM J. Math. Anal. 25 (1994), 876-893. Zbl 0806.35137 MR 1271315

8. Gurtin, M. E., Polignore, D., \& Viñals, J. Two-phase binary fluids and immiscible fluids described by an order parameter. Math. Models Methods Appl. Sci. 6 (1996), 815-831. Zbl 0857.76008 MR 1404829

9. HanzawA, E. Classical solutions of the Stefan problem. Tohoku Math. J. (2) 33 (1981), 297-335. Zbl 0571.35109 MR 0633045

10. KAто, T. Strong $L^{p}$-solutions of the Navier-Stokes equation in $\mathbb{R}^{m}$, with applications to weak solutions. Math. Z. 187 (1984), 471-480. Zbl 0545.35073 MR 0760047

11. Lemarié-RIeusset, P. G. Recent Developments in the Navier-Stokes Problem. Chapman \& Hall/CRC Res. Notes Math. 431, Chapman \& Hall/CRC, Boca Raton, FL (2002). Zbl 1034.35093 MR 1938147

12. LiU, C., \& SHEN, J. A phase field model for the mixture of two incompressible fluids and its approximation by a Fourier-spectral method. Phys. D 179 (2003), 211-228. Zbl 1092.76069 MR 1984386

13. LunARd, A. Analytic Semigroups and Optimal Regularity in Parabolic Problems. Progr. Nonlinear Differential Equations Appl. 16, Birkhäuser, Basel (1995). Zbl 0816.35001 MR 1329547

14. Maekawa, Y., \& Terasawa, Y. The Navier-Stokes equations with initial data in uniformly local $L^{p}$ spaces. Differential Integral Equations 19 (2006), 369-400. MR 2215625

15. Mogilevskil̆, I. Sh., \& Solonnikov, V. A. On the solvability of a free boundary problem for the Navier-Stokes equations in the Hölder space of functions. Nonlinear Analysis, Scuola Norm. Sup. Pisa Quaderni, Scuola Norm. Sup., Pisa (1991), 257-271. Z Zbl 0875.35063 MR 1205388

16. Nespoli, G., \& SAlvi, R. On the existence of two phase viscous incompressible flow. Advances in Fluid Dynamics, Quad. Mat. 4, Dept. Mat., Seconda Univ. Napoli, Caserta (1999), 245-268. Zbl 0949.35110 MR 1770191

17. Nouri, A., Poupaud, F., \& Demay, Y. An existence theorem for the multi-fluid Stokes problem. Quart. Appl. Math. 55 (1997), 421-435. Zbl 0882.35091 MR 1466141

18. Oseen, C. W. Neuere Methoden und Ergebnisse in der Hydrodynamik. Akademische Verlagsgesellschaft, Leipzig (1927). JFM 53.0773.02

19. Padula, M., \& Solonnikov, V. A. On the global existence of nonsteady motions of a fluid drop and their exponential decay to a uniform rigid rotation. Topics in Mathematical Fluid Mechanics, Quad. Mat. 10, Dept. Mat., Seconda Univ. Napoli, Caserta (2002), 185-218. Zbl 02079309 MR 2051775 
20. Plotnikov, P. I. Generalized solutions of a problem on the motion of a non-Newtonian fluid with a free boundary. Sibirsk. Mat. Zh. 34 (1993), no. 4, 127-141 (in Russian); English transl.: Siberian Math. J. 34 (1993), no. 4, 704-716. Zbl 0814.76007 MR 1248797

21. Runst, T., \& Sickel, W. Sobolev Spaces of Fractional Order, Nemytskij Operators, and Nonlinear Partial Differential Equations. De Gruyter Ser. Nonlinear Anal. Appl. 3, de Gruyter (1996). Zbl 0873.35001 MR 1419319

22. Shibata, Y., \& Shimizu, S. A decay property of the Fourier transform and its application to the Stokes problem. J. Math. Fluid Mech. 3 (2001), 213-230. Zbl 1102.35300 MR 1860123

23. Solonnikov, V. A. Lectures on evolution free boundary problems: classical solutions. Mathematical Aspects of Evolving Interfaces (Funchal, 2000), Lecture Notes in Math. 1812, Springer, Berlin (2003), 123-175. Zbl 1038.35063 MR 2011035

24. TANAKA, N. Global existence of two phase nonhomogeneous viscous incompressible fluid flow. Comm. Partial Differential Equations 18 (1993), 41-81. Zbl 0773.76073 MR 1211725

25. TANAKA, N. Two-phase free boundary problem for viscous incompressible thermocapillary convection. Japan. J. Math. (N.S.) 21 (1995), 1-42. Zbl 0845.35138 MR 1338355 